JOURNAL OF THE

AMERICAN MATHEMATICAL SOCIETY

Volume 18, Number 1, Pages 1-60

S 0894-0347(04)00472-2

Article electronically published on November 3, 2004

\title{
POLYLOGARITHMS, REGULATORS, AND ARAKELOV MOTIVIC COMPLEXES
}

\author{
A. B. GONCHAROV
}

\section{Contents}

1. Introduction

2. Arakelov motivic complexes

3. The Chow polylogarithms

4. The Grassmannian polylogarithms

5. Grassmannian polylogarithms, symmetric spaces and Borel regulators

6. The Chow dilogarithm and a reciprocity law

7. Appendix: On volumes of simplices in symmetric spaces

Acknowledgement

References

\section{INTRODUCTION}

Summary. We construct an explicit regulator map from the weight $n$ Bloch higher Chow group complex to the weight $n$ Deligne complex of a regular projective complex algebraic variety $X$. We define the weight $n$ Arakelov motivic complex as the cone of this map shifted by one. Its last cohomology group is (a version of) the Arakelov Chow group defined by H. Gillet and C. Soulé.

We relate the Grassmannian $n$-logarithms to the geometry of the symmetric space $S L_{n}(\mathcal{C}) / S U(n)$. For $n=2$ we recover Lobachevsky's formula expressing the volume of an ideal geodesic simplex in the hyperbolic space via the dilogarithm. Using the relationship with symmetric spaces we construct the Borel regulator on $K_{2 n-1}(\mathcal{C})$ via the Grassmannian $n$-logarithms.

We study the Chow dilogarithm and prove a reciprocity law which strengthens Suslin's reciprocity law for Milnor's group $K_{3}^{M}$ on curves.

Our note, "Chow polylogarithms and regulators" G5, can serve as an introduction to this paper.

1. Beilinson's conjectures on special values of $L$-functions. Let $X$ be a regular scheme. A. A. Beilinson [B1] defined the rational motivic cohomology of $X$

Received by the editors July 31, 2002.

2000 Mathematics Subject Classification. Primary 11G55, 19F27, 14G40, 19E15.

Key words and phrases. Polylogarithms, motivic complexes, regulators, Arakelov theory.

(C)2004 American Mathematical Society Reverts to public domain 28 years from publication 
via Quillen's algebraic $K$-theory of $X$ by the following formula:

$$
H_{\mathcal{M}}^{i}(X, \mathbb{Q}(n))=K_{2 n-i}^{(n)}(X)_{\mathbb{Q}}
$$

where the term on the right is the weight $n$ eigenspace for the Adams operations acting on $K_{2 n-i}(X) \otimes \mathbb{Q}$. For a regular complex algebraic variety $X$, Beilinson defined the regulator map to the weight $n$ Deligne cohomology of $X(\mathbb{C})$ :

$$
r_{B}: H_{\mathcal{M}}^{i}(X, \mathbb{Q}(n)) \longrightarrow H_{\mathcal{D}}^{i}(X(\mathbb{C}), \mathbb{R}(n)) .
$$

Let $X$ be a regular projective scheme over $\mathbb{Q}$. Then there is a $\mathbb{Q}$-vector subspace

$$
H_{\mathcal{M} / \mathbb{Z}}^{i}(X, \mathbb{Q}(n)) \subset H_{\mathcal{M}}^{i}(X, \mathbb{Q}(n)),
$$

called the integral part in the motivic cohomology; see [Sch]. Let $L\left(h^{i}(X), s\right)$ be the $L$-function related to its $i$-dimensional cohomology. Beilinson conjectured that for any integer $n>1+i / 2$ its special value at $s=n$ is described, up to a nonzero rational factor, by the regulator map to the weight $n$ real Deligne cohomology of $X$ :

$$
r_{B}: H_{\mathcal{M} / \mathbb{Z}}^{i+1}(X, \mathbb{Q}(n)) \longrightarrow H_{\mathcal{D}}^{i+1}\left(X \otimes \mathbb{Q} \mathbb{R}_{\mathbb{R}}, \mathbb{R}(n)\right) ;
$$

see [B1], $\mathrm{RSS}]$ or [N] for details.

This conjecture is fully established only when $X=\operatorname{Spec}(F)$ where $F$ is a number field. In this case the regulator map $r_{B}$ coincides, up to a nonzero rational factor, with the Borel regulator ([B1] $)$, and the relation with special values of the Dedekind zeta-function of $F$ was given by the Borel theorem [Bo2].

Although Beilinson's conjectures are far from being proved, it is interesting to see what kind of information concerning the special values of $L$-functions they suggest. So we come to the problem of explicit calculation of Beilinson's regulator. This problem is already very interesting for the Borel regulator.

2. Regulator maps on motivic complexes and Arakelov motivic cohomology. Beilinson [B2] and S. Lichtenbaum [Li] conjectured that the weight $n$ integral motivic cohomology of $X$ should appear as the cohomology of certain natural complexes of abelian groups $\Gamma(X ; n)$, called the weight $n$ motivic complexes:

$$
H_{\mathcal{M}}^{i}(X, \mathbb{Z}(n)):=H^{i}(\Gamma(X ; n)) .
$$

These complexes should calculate the hypercohomology of certain complexes of Zariski sheaves. The complexes $\Gamma(X ; n)$ are well-defined as objects of the derived category.

The first motivic complexes satisfying Beilinson's formula (11) were Bloch's higher Chow group complexes $\mathcal{Z} \bullet(X ; n)$ [B11]. Later on A.A. Suslin and V.A. Voevodsky defined several important versions of these complexes. For another candidate for motivic complexes, called the polylogarithmic motivic complexes, see [G1], G2. They are very explicit and the smallest among possible candidates; however, Beilinson's formula (1) is far from being established for them.

The real Deligne cohomology arises also as the cohomology of certain complexes. It was suggested in [G5] and G7 that the regulator map should be explicitly defined on the level of complexes.

Let $X$ be a regular projective variety over $\mathbb{C}$. In Section 2 we construct a homomorphism of complexes:

Bloch's weight $n$ higher Chow group complex $\mathcal{Z} \bullet(X ; n)$ of $X$

the weight $n$ real Deligne complex $\mathcal{C}_{\mathcal{D}}(X(\mathbb{C}) ; n)$ of $X$. 
This construction is a version of the one given in [G5]. The complex $\mathcal{C}_{\mathcal{D}}^{\bullet}(X(\mathbb{C}) ; n)$ is the truncation $\tau_{\leq 2 n}$ of the complex proposed by Deligne [Del]. The weight $n$ Arakelov motivic complex $\Gamma_{\mathcal{A}}^{\bullet}(X ; n)$ is defined as the cone of the map (3)), shifted by -1 :

$$
\Gamma_{\mathcal{A}}^{\bullet}(X ; n):=\operatorname{Cone}\left(\mathcal{Z}^{\bullet}(X ; n) \stackrel{3}{\longrightarrow} \mathcal{C}_{\mathcal{D}}^{\bullet}(X(\mathbb{C}) ; n)\right)[-1] .
$$

For a regular projective variety $X$ over $\mathbb{R}$, the image of map (33) lies in the subcomplex

$$
\mathcal{C}_{\mathcal{D}}^{\bullet}\left(X_{/ \mathbb{R}} ; n\right):=\mathcal{C}_{\mathcal{D}}^{\bullet}(X(\mathbb{C}) ; n)^{\bar{F}_{\infty}}
$$

where $\bar{F}_{\infty}$ is the de Rham involution provided by the action of complex conjugation. The weight $n$ real Arakelov motivic complex is defined as

$$
\Gamma_{\mathcal{A}}^{\bullet}(X / \mathbb{R} ; n):=\operatorname{Cone}\left(\mathcal{Z} \bullet(X ; n) \stackrel{(3)}{\longrightarrow} \mathcal{C}_{\mathcal{D}}^{\bullet}\left(X_{/ \mathbb{R}} ; n\right)\right)[-1] .
$$

Let $X$ be a regular projective variety $X$ over a number field $F$. We view $X$ over $\mathbb{Q}: X \longrightarrow \operatorname{Spec}(F) \longrightarrow \operatorname{Spec}(\mathbb{Q})$ and set

$$
\Gamma_{\mathcal{A}}^{\bullet}\left(X_{/ F} ; n\right):=\operatorname{Cone}\left(\mathcal{Z}^{\bullet}(X ; n) \longrightarrow \mathcal{C}_{\mathcal{D}}^{\bullet}\left(X \otimes_{\mathbb{Q}} \mathbb{R}_{\mathbb{R}} ; n\right)\right)[-1]
$$

The weight $n$ Arakelov motivic cohomology is the cohomology of this complex. Our construction works equally well for the Suslin-Voevodsky versions of the motivic complexes.

Taking the cohomology we get a construction of the regulator map on motivic cohomology. For a different construction, see [B13].

The regulator map on the polylogarithmic motivic complexes was defined in G7] explicitly via the classical polylogarithms. The Arakelov motivic complexes constructed using regulator maps on different motivic complexes are supposed to lead to the same object of the derived category. However, a precise relationship between the construction given in [G7] and the one in Section 2 is not clear.

Higher Arakelov Chow groups. The last group of the complex $\mathcal{C}_{\mathcal{D}}^{\bullet}(X(\mathbb{C}) ; n)$ consists of closed distributions of a certain type on $X(\mathbb{C})$. Replacing it by the quotient modulo smooth closed forms of the same type we get the quotient complex $\widetilde{\mathcal{C}}_{\mathcal{D}}(X(\mathbb{C}) ; n)$. Changing $\mathcal{C}$ to $\widetilde{\mathcal{C}}$ in (44) we define the weight $n$ higher Arakelov Chow group complex. Its last cohomology group is isomorphic to the Arakelov Chow group $\widehat{C H}^{n}(X(\mathbb{C}))$ as defined by Gillet and Soulé [GS], [S].

Problems. (a) Show that taking cohomology of the map (3) and using the isomorphism between the rational Bloch's higher Chow groups of $X$ and the corresponding part of the rational $K$-theory of $X([\mathrm{Bl} 2],[\mathrm{Lev}])$ we get a nonzero rational multiple of Beilinson's regulator map.

(b) Generalize the arithmetic Riemann-Roch theorem proved by Gillet and Soulé to the case of higher Arakelov Chow groups.

Remark. The weight $n$ Arakelov motivic complex should be considered as an ingredient of a definition of the weight $n$ arithmetic motivic complex. The latter is related to the regulator maps on $H_{\mathcal{M} / \mathbb{Z}}^{\bullet}(X, \mathbb{Q}(n))$, while the former is related to the ones on $H_{\mathcal{M}}(X, \mathbb{Q}(n))$. Ideally one should have for every place $p$ of $\mathbb{Q}$ a map from the left-hand side of (3) to a certain complex, which for the Archimedean place should be given by our map. Then one should take the shifted by -1 cone of the sum of these maps. 
3. The Chow $n$-logarithm function. Let us describe the regulator map (3) in the simplest case when $X=\operatorname{Spec}(\mathbb{C})$ is a point.

Let us choose in $\mathbb{P}^{m}$ homogeneous coordinates $\left(z_{0}: \ldots: z_{m}\right)$. The union of the coordinate hyperplanes is a simplex $L$. Let $\mathbb{A}^{m}$ be the complement to the hyperplane $z_{1}+\ldots+z_{m}=z_{0}$ in $\mathbb{P}^{m}$. The abelian group $\mathcal{Z}_{m}(\operatorname{Spec}(\mathbb{C}) ; n)$ is freely generated by the codimension $n$ irreducible algebraic cycles in $\mathbb{A}^{m}$ intersecting properly the faces of the simplex $L$. The intersection with codimension one faces $L_{j}$ of $L$ provides homomorphisms

$$
\partial_{j}: \mathcal{Z}_{m}(\operatorname{Spec}(\mathbb{C}) ; n) \longrightarrow \mathcal{Z}_{m-1}(\operatorname{Spec}(\mathbb{C}) ; n) ; \quad \partial:=\sum_{j=0}^{m}(-1)^{j} \partial_{j}
$$

The weight $n$ higher Chow group complex over $\operatorname{Spec}(\mathbb{C})$, where $n>0$, written as a homological complex, looks as follows:

$$
\ldots \stackrel{\partial}{\longrightarrow} \mathcal{Z}_{2}(\operatorname{Spec}(\mathbb{C}) ; n) \stackrel{\partial}{\longrightarrow} \mathcal{Z}_{1}(\operatorname{Spec}(\mathbb{C}) ; n) \stackrel{\partial}{\longrightarrow} \mathcal{Z}_{0}(\operatorname{Spec}(\mathbb{C}) ; n) .
$$

The Deligne complex of a point is the complex $(2 \pi i)^{n} \mathbb{R} \longrightarrow \mathbb{C}$, where $\mathbb{C}$ is in the degree +1 . So it is quasi-isomorphic to the group $\mathbb{R}(n-1):=(2 \pi i)^{n-1} \mathbb{R}$ placed in degree +1 . The regulator map (3) boils down to a construction of a homomorphism

$$
\mathcal{Z}_{2 n-1}(\operatorname{Spec}(\mathbb{C}) ; n) \stackrel{\mathcal{P}_{n}}{\longrightarrow} \mathbb{R}(n-1), \quad \text { such that } \mathcal{P}_{n} \circ \partial=0 .
$$

It is provided by a function $\mathcal{P}_{n}$ on the space of codimension $n$ cycles in $\mathbb{C P}^{2 n-1}$ intersecting properly the faces of a simplex $L$. This function, called the Chow $n$ logarithm function, was constructed in G5]. To recall its construction, observe that a codimension $n$ cycle given by an irreducible subvariety $X$ in $\mathbb{P}^{2 n-1}-L$ provides the $(n-1)$-dimensional variety $X$ with $2 n-1$ rational functions $f_{1}, \ldots, f_{2 n-1}$. These functions are obtained by restriction of the coordinate functions $z_{i} / z_{0}$ to the cycle $X$. We define a natural $(2 n-2)$-form $r_{2 n-2}\left(f_{1}, \ldots, f_{2 n-1}\right)$ on $X(\mathbb{C})$ and set

$$
\mathcal{P}_{n}\left(X ; f_{1}, \ldots, f_{2 n-1}\right):=(2 \pi i)^{1-n} \int_{X(\mathbb{C})} r_{2 n-2}\left(f_{1}, \ldots, f_{2 n-1}\right) .
$$

4. An example: the Chow dilogarithm. Let $f_{1}, f_{2}, f_{3}$ be three arbitrary rational functions on a complex curve $X$. Set

$$
r_{2}\left(f_{1}, f_{2}, f_{3}\right):=\operatorname{Alt}_{3}\left(\frac{1}{6} \log \left|f_{1}\right| d \log \left|f_{2}\right| \wedge d \log \left|f_{3}\right|-\frac{1}{2} \log \left|f_{1}\right| d \arg f_{2} \wedge d \arg f_{3}\right)
$$

where $\mathrm{Alt}_{3}$ is the alternation of $f_{1}, f_{2}, f_{3}$. Consider the space of quadruples $\left(X ; f_{1}\right.$, $\left.f_{2}, f_{3}\right)$. It is a union of finite-dimensional algebraic varieties. The Chow dilogarithm is a real function on its complex points defined by the formula

$$
\mathcal{P}_{2}\left(X ; f_{1}, f_{2}, f_{3}\right):=\frac{1}{2 \pi i} \int_{X(\mathbb{C})} r_{2}\left(f_{1}, f_{2}, f_{3}\right) .
$$

The integral converges. The Chow dilogarithm provides a homomorphism

$$
\Lambda^{3} \mathbb{C}(X)^{*} \rightarrow \mathbb{R}, \quad f_{1} \wedge f_{2} \wedge f_{3} \longmapsto \mathcal{P}_{2}\left(X ; f_{1}, f_{2}, f_{3}\right) .
$$

Why does the dilogarithm appear in the name of the function $\mathcal{P}_{2}$ ? Recall the classical dilogarithm

$$
L i_{2}(z):=-\int_{0}^{z} \log (1-z) d \log z
$$

It has a single-valued cousin, the Bloch-Wigner function:

$$
\mathcal{L}_{2}(z):=\operatorname{Im} L i_{2}(z)+\arg (1-z) \log |z| \text {. }
$$


The Chow dilogarithm is defined by a two-dimensional integral over $X(\mathbb{C})$, while $\mathcal{L}_{2}(z)$ is given by an integral over a path in $\mathbb{C P}^{1}$. In Section 6 we show that nevertheless the Chow dilogarithm can be expressed by the function $\mathcal{L}_{2}(z)$. Here is how it works when $X=\mathbb{C P}^{1}$. For $f \in \mathbb{C}(X)$ let $v_{x}(f)$ be the order of zero of $f$ at $x \in X(\mathbb{C})$. Choose a point $\infty$ on $\mathbb{P}^{1}$. Then

$$
\mathcal{P}_{2}\left(\mathbb{C P}^{1} ; f_{1}, f_{2}, f_{3}\right)=\sum_{x_{i} \in \mathbb{P}^{1}(\mathbb{C})} v_{x_{1}}\left(f_{1}\right) v_{x_{2}}\left(f_{2}\right) v_{x_{3}}\left(f_{3}\right) \mathcal{L}_{2}\left(r\left(x_{1}, x_{2}, x_{3}, \infty\right)\right)
$$

where $r(\ldots)$ denotes the cross-ratio of four points on $\mathbb{P}^{1}$. A formula for the Chow dilogarithm on elliptic curves is given in Section 6 .

The function $\mathcal{L}_{2}$ satisfies Abel's five term functional equation:

$$
\sum_{i=1}^{5}(-1)^{i} \mathcal{L}_{2}\left(r\left(x_{1}, \ldots, \widehat{x}_{i}, \ldots, x_{5}\right)\right)=0 .
$$

The Chow dilogarithm also satisfies functional equations. They appear as a reformulation of the fact that the composition

$$
\mathcal{Z}_{4}(\operatorname{Spec}(\mathbb{C}) ; 2) \stackrel{\partial}{\longrightarrow} \mathcal{Z}_{3}(\operatorname{Spec}(\mathbb{C}) ; 2) \stackrel{\mathcal{P}_{2}}{\longrightarrow} \mathbb{R}(1)
$$

is zero. Namely, let $Y$ be an algebraic surface with four rational functions $g_{1}, \ldots, g_{4}$ on it corresponding to an element of $\mathcal{Z}_{4}(\operatorname{Spec}(\mathbb{C}) ; 2)$. To evaluate the composition on this element we do the following. Take the $\operatorname{divisor} \operatorname{div}\left(g_{i}\right)$ and restrict the other functions $g_{i}$ to it. Then applying the Chow dilogarithm to the obtained data and taking the alternating sum over $1 \leq i \leq 4$ we get zero. In the special case when $Y=\mathbb{C P}^{2}$ and $\operatorname{div} g_{i}=\left(l_{i}\right)-\left(l_{5}\right)$, where $l_{1}, \ldots, l_{5}$ are five lines in the plane, this functional equation plus (9) is equivalent to Abel's equation (10).

5. The Grassmannian $n$-logarithm and symmetric space $S L_{n}(\mathbb{C}) / S U(n)$. Restricting the Chow $n$-logarithm function to the subvariety of $(n-1)$-planes in $\mathbb{C P}^{2 n-1}$ in general position with respect to the simplex $L$ we get the Grassmannian $n$-logarithm function $\mathcal{L}_{n}^{G}$.

Let $G$ be a group and $X$ a $G$-set. Configurations of $n$ points in $X$ are by definition the points of the quotient $X^{n} / G$. There is a natural bijection

$$
\begin{aligned}
& \left\{(n-1) \text {-planes in } \mathbb{P}^{2 n-1}\right. \text { in generic position } \\
& \text { with respect to a simplex } L\} /\left(\mathbb{G}_{m}^{*}\right)^{2 n-1} \\
<-->\quad\left\{\text { Configurations of } 2 n \text { generic hyperplanes in } \mathbb{P}^{n-1}\right\} &
\end{aligned}
$$

given by intersecting an $(n-1)$-plane $h$ with the codimension one faces of $L$. (See Figure (1).

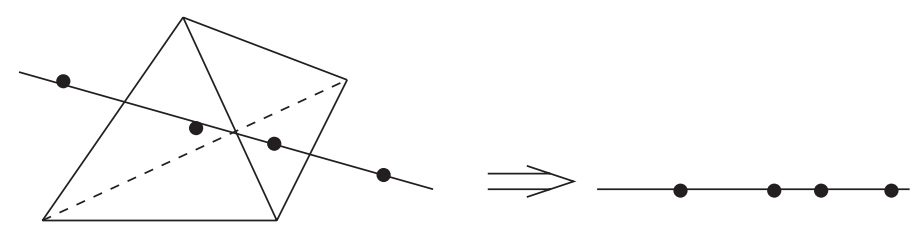

FIGURE 1. Toric quotients of Grassmannians and configurations of hyperplanes 
Using this bijection we can view $\mathcal{L}_{n}^{G}$ as a function on the configurations of $2 n$ hyperplanes in $\mathbb{C P}^{n-1}$. Applying the projective duality we can consider it as a function on configurations of $2 n$ points in $\mathbb{C P}^{n-1}$.

In fact, one can define the Grassmannian $n$-logarithm $\mathcal{L}_{n}^{G}\left(x_{1}, \ldots, x_{2 n}\right)$ as a function on configurations of arbitrary $2 n$ points in $\mathbb{C P}^{n-1}$; see Section 4 . It is a measurable function which is real analytic on generic configurations. It satisfies the two functional equations

$$
\sum_{i=0}^{2 n}(-1)^{i} \mathcal{L}_{n}^{G}\left(x_{0}, \ldots, \widehat{x}_{i}, \ldots, x_{2 n}\right)=0, \quad \sum_{j=0}^{2 n}(-1)^{j} \mathcal{L}_{n}^{G}\left(y_{j} \mid y_{0}, \ldots, \widehat{y}_{j}, \ldots, y_{2 n}\right)=0 .
$$

In the second formula $\left(y_{0}, \ldots, y_{2 n}\right)$ is a configuration of $2 n+1$ points in $\mathbb{C P}^{n}$ and $\left(y_{j} \mid y_{0}, \ldots, \widehat{y}_{j}, \ldots, y_{2 n}\right)$ is a configuration of $2 n$ points in $\mathbb{C P}^{n-1}$ obtained by projection from $y_{j}$. (See Figure 2)

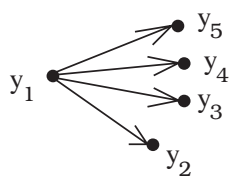

Figure 2. The configuration $\left(y_{1} \mid y_{2}, y_{3}, y_{4}, y_{5}\right)$ on $\mathbb{P}^{1}$

It follows from (9) that the Grassmannian dilogarithm is given by the BlochWigner function:

$$
\mathcal{L}_{2}^{G}\left(z_{1}, \ldots, z_{4}\right)=\mathcal{L}_{2}\left(r\left(z_{1}, \ldots, z_{4}\right)\right) .
$$

Abel's five term equation coincides with (11). (The two functional equations (11) are equivalent when $n=2$.)

Lobachevsky discovered that the dilogarithm appears in the computation of volumes of geodesic simplices in the three-dimensional hyperbolic space $\mathcal{H}_{3}$. Let $I\left(z_{1}, \ldots, z_{4}\right)$ be the ideal geodesic simplex with vertices at the points $z_{1}, \ldots, z_{4}$ on the absolute of $\mathcal{H}_{3}$. (See Figure 3) The absolute is naturally identified with $\mathbb{C P}^{1}$. Lobachevsky's formula relates its volume to the Bloch-Wigner function:

$$
\operatorname{vol}\left(I\left(z_{1}, \ldots, z_{4}\right)\right)=\mathcal{L}_{2}\left(r\left(z_{1}, \ldots, z_{4}\right)\right)
$$

The volume function $\operatorname{vol} I\left(z_{1}, \ldots, z_{4}\right)$ is invariant under the group $S L_{2}(\mathbb{C})$ of isometries of $\mathcal{H}_{3}$. So it depends only on the cross-ratio of the points $z_{1}, \ldots, z_{4}$. It satisfies the five term equation (10). Indeed, $\sum(-1)^{i} I\left(z_{1}, \ldots, \widehat{z}_{i}, \ldots, z_{5}\right)=\emptyset$. By Bloch's theorem [B12] any measurable function $f(z)$ on $\mathbb{C}$ satisfying the five term equation is proportional to $\mathcal{L}_{2}(z)$. So we get the formula up to a constant.

We generalize this picture as follows. $\mathbb{C P}^{n-1}$ is realized as the smallest boundary stratum of the symmetric space $\mathbb{H}_{n}:=S L_{n}(\mathbb{C}) / S U(n)$. We define a function $\psi_{n}\left(x_{1}, \ldots, x_{2 n}\right)$ on configurations of $2 n$ points of the symmetric space. The function $\psi_{n}$ is defined by an integral over $\mathbb{C P}^{n-1}$ similar to (17). We show that it can be naturally extended to a function $\bar{\psi}_{n}$ on configurations of $2 n$ points in a compactification $\overline{\mathbb{H}}_{n}$ of the symmetric space. The Grassmannian $n$-logarithm function turns out to be the value of the function $\bar{\psi}_{n}$ on configurations of $2 n$ points at the smallest boundary strata, which is identified with $\mathbb{C P}^{n-1}$. 


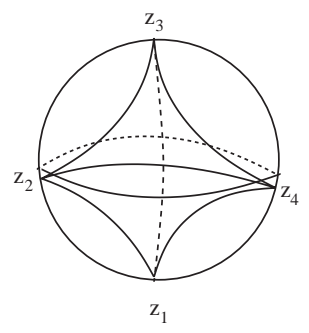

Figure 3. An ideal simplex in the hyperbolic 3-space

Now let $n=2$. Then $S L_{2}(\mathbb{C}) / S U(2)$ is identified with the hyperbolic 3 -space. We prove in Section 7 that $\psi_{2}\left(x_{1}, x_{2}, x_{3}, x_{4}\right)$ is the volume of the geodesic simplex with vertices at the points $x_{1}, \ldots, x_{4}$. Restricting to the ideal geodesic simplices and using the relation to the Grassmannian dilogarithm plus (12) we get a new proof of Lobachevsky's formula.

6. The Grassmannian $n$-logarithms and the Borel regulator. For any point $x \in \mathbb{C P}^{n-1}$ the function

$$
c_{2 n-1}^{n}\left(g_{1}, \ldots, g_{2 n}\right):=\mathcal{L}_{n}^{G}\left(g_{1} x, \ldots, g_{2 n} x\right)
$$

is a measurable $(2 n-1)$-cocycle of the Lie group $G L_{n}(\mathbb{C})$. Indeed, it is invariant under the diagonal action of $G L_{n}(\mathbb{C})$, and the cocycle condition is just the first functional equation for the function $\mathcal{L}_{n}^{G}$. Different points $x$ give canonically cohomologous cocycles. However, a priori it is not clear that the corresponding cohomology class is non-zero.

Let $H_{m}^{2 n-1}\left(G L_{n}(\mathbb{C}), \mathbb{R}\right)$ be the space of measurable cohomology of the Lie group $G L_{n}(\mathbb{C})$. It is known that

$$
H_{m}^{*}\left(G L_{n}(\mathbb{C}), \mathbb{R}\right)=\Lambda_{\mathbb{R}}^{*}\left(b_{1}, b_{3}, \ldots, b_{2 n-1}\right)
$$

where $b_{2 k-1} \in H_{m}^{2 k-1}\left(G L_{n}(\mathbb{C}), \mathbb{R}\right)$ are certain canonical generators called the Borel classes ([Bo1]).

Theorem 1.1. The cohomology class of the Grassmannian cocycle (13) is a nonzero rational multiple of the Borel class $b_{2 n-1}$.

For normalization of the Borel classes and the precise relationship between the Grassmannian polylogarithms and the Borel regulator, see Section 5, especially Subsections 4 and 5 .

The essential role in the proof is played by the fact that the Grassmannian $n$-logarithm function $\mathcal{L}_{n}^{G}$ is a boundary value of the function $\bar{\psi}_{n}$. The function $\bar{\psi}_{n}\left(x_{1}, \ldots, x_{2 n}\right)$ is not continuous at certain boundary points, but always satisfies the cocycle condition. So taking any point $x \in \overline{\mathbb{H}}_{n}$ we get a cocycle

$$
c_{x}\left(g_{1}, \ldots, g_{2 n-1}\right):=\bar{\psi}_{n}\left(g_{1} x, \ldots, g_{2 n} x\right)
$$

of the group $G L_{n}(\mathbb{C})$. Its cohomology class does not depend on $x$. If $x \in \mathbb{H}_{n}$ the corresponding cocycle is smooth. We can differentiate it, getting a cohomology class of the Lie algebra $g l_{n}$, and relate it to the Borel class. On the other hand, taking $x$ to be a point on the boundary stratum $\mathbb{C P}^{n-1}$ we recover the Grassmannian cocycle (13). So we get the theorem. 
Combining it with the technique developed in [G1], G2], we get a simple explicit construction of the Borel regulator

$$
K_{2 n-1}(\mathbb{C}) \longrightarrow \mathbb{R}
$$

in terms of the Grassmannian $n$-logarithms. The second functional equation for $\mathcal{L}_{n}^{G}$ plays an important role in the proof. Therefore, thanks to the Borel theorem Bo2], this allows us to express the special values of Dedekind $\zeta$-functions at $s=n$ via the Grassmannian $n$-logarithms.

The definition of the higher Chow groups of a variety $X$ is much simpler than the definition of algebraic $K$-groups of $X$. The situation with the regulator maps is similar. However, relating the special values of the Dedekind $\zeta$-functions to motivic cohomology of the corresponding number fields, we need to work with the algebraic $K$-theory (or homology of $G L_{n}(F)$ ) of number fields.

Section 2 is the main core of the paper. In Sections 3, 4 and 6 the main construction of Section 2 is investigated from different points of view. Sections 4 and 5 are rather independent from the other sections.

\section{Arakelov motivic COMPleXes}

1. The higher Chow group complex. A (nondegenerate) simplex in $\mathbb{P}^{m}$ is an ordered collection of hyperplanes $L_{0}, \ldots, L_{m}$ in generic position, i.e., with empty intersection. Let us choose in $\mathbb{P}^{m}$ a simplex $L$ and a generic hyperplane $H$. We might think about this data as of a simplex in the $m$-dimensional affine space $\mathbb{A}^{m}:=\mathbb{P}^{m}-H$. For any two nondegenerate simplices in $\mathbb{A}^{m}$ there is a unique affine transformation sending one simplex to the other.

Let $I=\left(i_{1}, \ldots, i_{k}\right)$ and $L_{I}:=L_{i_{1}} \cap \ldots \cap L_{i_{k}}$. Let $X$ be a regular projective variety over a field $F$. Let $\mathcal{Z}_{m}(X ; n)$ be the free abelian group generated by irreducible codimension $n$ algebraic subvarieties in $X \times \mathbb{A}^{m}$ which intersect properly (i.e., with the right codimension) all faces $X \times L_{I}$.

Warning. We use the notation $\mathcal{Z}_{m}(X ; n)$ for the group denoted $\mathcal{Z}^{n}(X ; m)$ by Bloch. This allows us to use upper and lower indices to distinguish between the homological and cohomological notation; see below.

For a given codimension 1 face $L_{i}$ of a simplex $L$ in $\mathbb{A}^{m}$ the other faces $L_{j}$ cut a simplex $\widehat{L}_{i}:=\left\{L_{i} \cap L_{j}\right\}$ in $L_{i}$. So the intersection with codimension 1 faces $X \times L_{i}$ provides group homomorphisms

$$
\partial_{i}: \mathcal{Z}_{m}(X ; n) \longrightarrow \mathcal{Z}_{m-1}(X ; n) ; \quad \partial:=\sum_{i=0}^{m}(-1)^{i} \partial_{i} .
$$

Then $\partial^{2}=0$, so $\left(\mathcal{Z}_{\bullet}(X ; n) ; \partial\right)$ is a homological complex. Its homology groups are Bloch's higher Chow groups. By the fundamental theorem of Bloch ([B11], BB12], Lev )

$$
H_{i}\left(\mathcal{Z}_{\bullet}(X ; n) \otimes \mathbb{Q}\right)=K_{i}^{(n)}(X)_{\mathbb{Q}} .
$$

Let us cook up a cohomological complex by setting

$$
\mathcal{Z}^{\bullet}(X ; n):=\mathcal{Z}_{2 n-\bullet}(X ; n) .
$$

Its cohomology provides a definition of the integral motivic cohomology of $X$ :

$$
H_{\mathcal{M}}^{i}(X, \mathbb{Z}(n)):=H^{i}\left(\mathcal{Z}^{\bullet}(X ; n)\right) .
$$


Bloch's theorem guarantees Beilinson's formula (1) for the rational motivic cohomology.

2. The Beilinson-Deligne complex. Recall that an $n$-distribution, sometimes also called an $n$-form with generalized function coefficients or an $n$-current, on a smooth oriented manifold $X$ is a continuous linear functional on the space of $\left(\operatorname{dim}_{\mathbb{R}} X-n\right)$-forms with compact support. Denote by $\mathcal{D}_{X}^{n}$ the space of all real $n$-distributions on $X$. Let $\underline{\mathcal{D}}_{X}^{n}$ be the corresponding complex of sheaves on $X(\mathbb{C})$. The space $\mathcal{A}_{X}^{n}$ of all smooth $n$-forms on $X$ is a subspace of $\mathcal{D}_{X}^{n}$. The de Rham complex of distributions $\left(\underline{\mathcal{D}}_{X}^{\bullet}, d\right)$ is a resolution of the constant sheaf $\mathbb{R}$.

Let $X$ be a regular projective variety over $\mathbb{C}$. The standard weight $n$ BeilinsonDeligne complex $\mathbb{R}^{\bullet}(X ; n)_{\mathcal{D}}$ is the total complex associated with the following bicomplex of sheaves in the classical topology on $X(\mathbb{C})$ :

$$
\begin{aligned}
&\left(\underline{\mathcal{D}}_{X}^{0} \stackrel{d}{\longrightarrow} \underline{\mathcal{D}}_{X}^{1} \stackrel{d}{\longrightarrow} \ldots \stackrel{d}{\longrightarrow} \underline{\underline{D}}_{X}^{n} \stackrel{\stackrel{d}{\longrightarrow}}{\longrightarrow} \underline{\mathcal{D}}_{X}^{n+1} \stackrel{d}{\longrightarrow} \ldots\right) \otimes \mathbb{R}(n-1) \\
& \uparrow \pi_{n} \stackrel{\uparrow \pi_{n}}{\longrightarrow} \\
& \Omega_{X}^{n} \stackrel{\partial}{\longrightarrow} \Omega_{X}^{n+1} \stackrel{\partial}{\longrightarrow}
\end{aligned}
$$

Here $\mathbb{R}(n):=(2 \pi i)^{n} \mathbb{R}$ and

$$
\pi_{n}: \underline{\mathcal{D}}_{X}^{p} \otimes \mathbb{C} \longrightarrow \underline{\mathcal{D}}_{X}^{p} \otimes \mathbb{R}(n-1)
$$

is the projection induced by the one $\mathbb{C}=\mathbb{R}(n-1) \oplus \mathbb{R}(n) \longrightarrow \mathbb{R}(n-1)$. Further, $\underline{\mathcal{D}}_{X}^{0}$ placed in degree 1 and $\left(\Omega_{X}^{\bullet}, \partial\right)$ is the de Rham complex of holomorphic forms.

The Beilinson-Deligne complex $\mathbb{R}^{\bullet}(X ; n)_{\mathcal{D}}$ is quasi-isomorphic to the complex of sheaves

$$
\underline{\mathbb{R}}(n) \longrightarrow \mathcal{O}_{X} \longrightarrow \Omega_{X}^{1} \longrightarrow \Omega_{X}^{2} \longrightarrow \ldots \longrightarrow \Omega_{X}^{n-1} .
$$

3. The truncated Deligne complex. Let $\mathcal{D}_{X}^{p, q}=\mathcal{D}^{p, q}$ be the abelian group of complex-valued distributions of type $(p, q)$ on $X(\mathbb{C})$. Consider the following cohomological "bicomplex", where $\mathcal{D}_{\mathrm{cl}}^{n, n}$ is the subspace of the space $\mathcal{D}^{n, n}$ of closed currents, and $\mathcal{D}^{0,0}$ is in degree 1 :

$$
\mathcal{D}_{c l}^{n, n}
$$

$$
\begin{array}{ccccccc}
\mathcal{D}^{0, n-1} & \stackrel{\partial}{\longrightarrow} & \mathcal{D}^{1, n-1} & \stackrel{\partial}{\longrightarrow} & \ldots & \stackrel{\partial}{\longrightarrow} & \mathcal{D}^{n-1, n-1} \\
\bar{\partial} \uparrow & & \bar{\partial} \uparrow & & & & \bar{\partial} \uparrow \\
\ldots & \ldots & \ldots & \ldots & \ldots & \ldots & \ldots \\
\bar{\partial} \uparrow & & \bar{\partial} \uparrow & & & & \bar{\partial} \uparrow \\
\mathcal{D}^{0,1} & \stackrel{\partial}{\longrightarrow} & \mathcal{D}^{1,1} & \stackrel{\partial}{\longrightarrow} & \ldots & \stackrel{\partial}{\longrightarrow} & \mathcal{D}^{n-1,1} \\
\bar{\partial} \uparrow & & \bar{\partial} \uparrow & & & & \bar{\partial} \uparrow \\
\mathcal{D}^{0,0} & \stackrel{\partial}{\longrightarrow} & \mathcal{D}^{1,0} & \stackrel{\partial}{\longrightarrow} & \ldots & \stackrel{\partial}{\longrightarrow} & \mathcal{D}^{n-1,0}
\end{array}
$$


Properly speaking, it is not a bicomplex due to the presence of the operator $2 \bar{\partial} \partial$, but we can handle it the same way we handle the bicomplexes. Namely, we define its total complex $T_{o t} t^{\bullet}$. It is concentrated in degrees $[1,2 n]$. The complex $C_{\mathcal{D}}^{\bullet}(X(\mathbb{C}) ; n)=C_{\mathcal{D}}^{\bullet}(n)$ is a subcomplex of the complex $T^{\bullet} t^{\bullet}$ defined as follows. Take the intersection of the part of the complex $T^{\circ} t^{\bullet}$ coming from the $n \times n$ square in the diagram (and concentrated in degrees [1,2n-1]) with the complex of distributions with values in $\mathbb{R}(n-1)$. Consider the subgroup $\mathcal{D}_{\mathbb{R}, c l}^{n, n}(n) \subset \mathcal{D}_{c l}^{n, n}$ of the $\mathbb{R}(n)$-valued distributions of type $(n, n)$. They form a subcomplex in $T o t^{\bullet}$ because $\bar{\partial} \partial$ sends $\mathbb{R}(n-1)$-valued distributions to $\mathbb{R}(n)$-valued distributions. This is the complex $C_{\mathcal{D}}^{\bullet}(n)$. It is a truncation of the complex considered by Deligne ([Del] $)$. Its cohomology is isomorphic to the absolute Hodge cohomology defined by Beilinson [B3].

Let us replace the complex $\Omega_{X}^{n+\bullet}$ in (14) by its Dolbeault resolution. We get a bicomplex of sheaves. Denote by $\mathcal{R}^{\bullet}(X ; n)_{\mathcal{D}}$ the complex of global sections of the total complex of this bicomplex.

Proposition 2.1. Let $X$ be a regular complex projective variety. Then the complex $C_{\mathcal{D}}^{\bullet}(X ; n)$ is quasi-isomorphic to the truncated complex $\tau_{\leq 2 n} \mathcal{R} \bullet(X ; n)_{\mathcal{D}}$.

Proof. We need the following general construction. Let $f^{\bullet}: X^{\bullet} \longrightarrow Y^{\bullet}$ be a morphism of complexes such that the map $f^{i}$ is injective for $i \leq p$ and surjective for $i \geq p$ (and hence is an isomorphism for $i=p$ ). Consider a complex

$$
Z^{\bullet}:=\operatorname{Coker} f^{<p}[-1] \stackrel{D}{\longrightarrow} \operatorname{Ker} f^{>p}
$$

where the differential $D: \operatorname{Coker} f^{p-1} \longrightarrow \operatorname{Ker} f^{p+1}[1]$ is defined via the following diagram (the vertical sequences are exact):

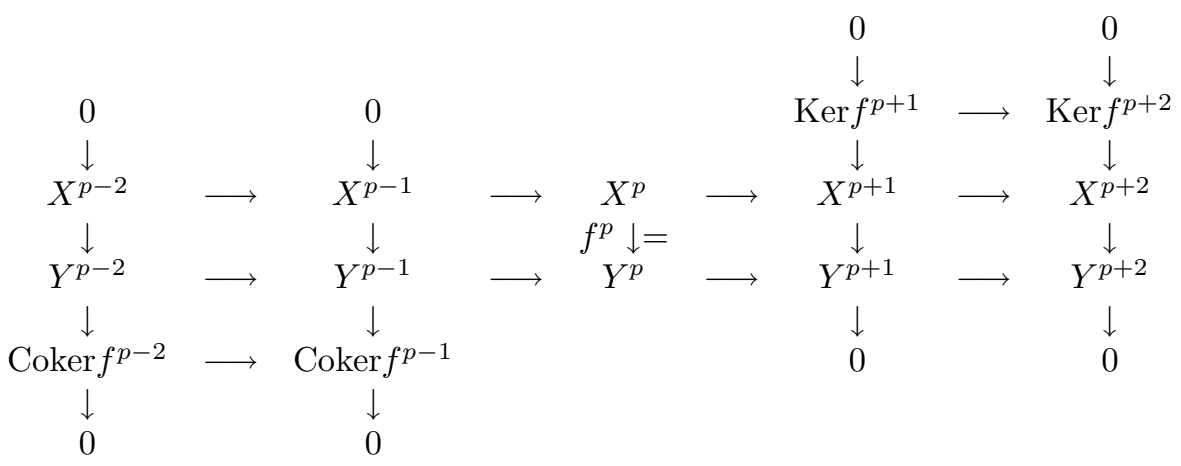

Lemma 2.2. The complex $Z^{\bullet}$ is canonically quasi-isomorphic to $\operatorname{Cone}\left(X^{\bullet} \stackrel{f^{\bullet}}{\longrightarrow}\right.$ $\left.Y^{\bullet}\right)$.

Proof. Let

$$
\begin{gathered}
\widetilde{\tau}_{<p} X^{\bullet}:=\quad \ldots \stackrel{d_{X}}{\longrightarrow} X^{p-2} \stackrel{d_{X}}{\longrightarrow} X^{p-1} \stackrel{d_{X}}{\longrightarrow} \operatorname{Im} d_{X} \\
\widetilde{\tau}_{\geq p} Y^{\bullet}:=\quad Y^{p} / \operatorname{Im} d_{Y} \stackrel{d_{Y}}{\longrightarrow} Y^{p+1} \stackrel{d_{Y}}{\longrightarrow} Y^{p+2} \stackrel{d_{Y}}{\longrightarrow} \ldots
\end{gathered}
$$


Then there is an exact sequence of complexes $0 \longrightarrow \widetilde{\tau}_{<p} X^{\bullet} \longrightarrow X^{\bullet} \longrightarrow \widetilde{\tau}_{\geq p} X^{\bullet} \longrightarrow$ 0 . The conditions on the maps $f^{\bullet}$ imply that

$$
\begin{array}{cc}
\widetilde{\tau}_{<p} f^{\bullet}: & \widetilde{\tau}_{<p} X^{\bullet} \longrightarrow \widetilde{\tau}_{<p} Y^{\bullet} \quad \text { is injective, } \\
\widetilde{\tau}_{\geq p} f^{\bullet}: & \widetilde{\tau}_{\geq p} Y^{\bullet} \longrightarrow \widetilde{\tau}_{\geq p} Y^{\bullet} \quad \text { is surjective. }
\end{array}
$$

We get maps of complexes

$$
\operatorname{Cone}\left(\widetilde{\tau}_{<p} X^{\bullet} \longrightarrow f^{\bullet}\left(\widetilde{\tau}_{<p} X^{\bullet}\right)\right) \stackrel{\alpha}{\hookrightarrow} \operatorname{Cone}\left(X^{\bullet} \longrightarrow Y^{\bullet}\right) \stackrel{\beta}{\longrightarrow} \operatorname{Cone}\left(\widetilde{\tau}_{\geq p} Y^{\bullet} \longrightarrow \widetilde{\tau}_{\geq p} Y^{\bullet}\right)
$$

where $\alpha$ is injective and $\beta$ is surjective. The complex $\operatorname{Ker}(\beta) / \operatorname{Im}(\alpha)$ looks as follows:

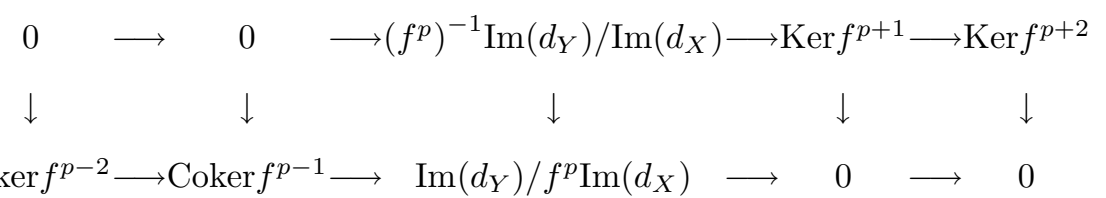

Since the map $f^{p}: \operatorname{Im}\left(d_{Y}\right) / \operatorname{Im}\left(d_{X}\right) \rightarrow \operatorname{Im}\left(d_{Y}\right) / f^{p} \operatorname{Im}\left(d_{X}\right)$ is an isomorphism it is quasi-isomorphic to $Z \bullet$. The lemma is proved.

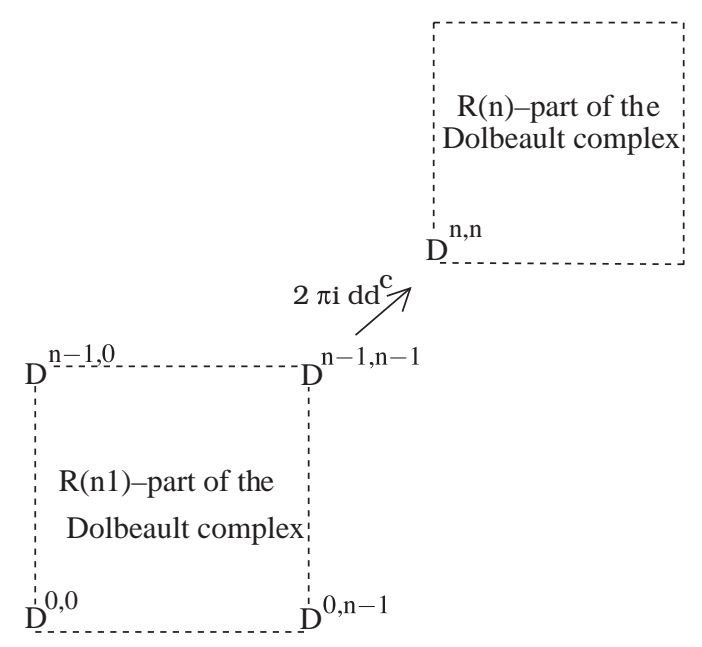

Figure 4 . The weight $n$ real Deligne complex

Let us apply Lemma 2.2 to the morphism of complexes

$$
\operatorname{Tot}\left(\mathcal{D}^{\geq n, \bullet}\right) \stackrel{\pi_{n}}{\longrightarrow} \mathcal{D}^{\bullet} \otimes_{\mathbb{R}} \mathbb{R}(n-1) .
$$

We claim that it implies that the complex $\mathbb{R}(n)_{\mathcal{D}}$ is canonically quasi-isomorphic to the complex shown in Figure4. To prove this it remains to compute the differential

$$
\mathcal{D}_{\mathbb{R}}^{n-1, n-1}(n-1) \longrightarrow \mathcal{D}_{\mathbb{R}}^{n, n}(n) .
$$

We proceed as follows. Take $\alpha \in \mathcal{D}_{\mathbb{R}}^{n-1, n-1}(n-1)$, so $\alpha=(-1)^{n-1} \bar{\alpha}$. Then

$$
d \alpha=\partial \alpha+\bar{\partial} \alpha=\partial \alpha+(-1)^{n-1} \overline{\partial \alpha}=2 \pi_{n}(\partial \alpha) .
$$

Applying $d=\partial+\bar{\partial}$ again and taking the $(n, n)$-component we get $2 \bar{\partial} \partial(\alpha)$. Truncating this complex we obtain the proof of the proposition. (Note that $d^{C}:=$ $(4 \pi i)^{-1}(\partial-\bar{\partial})$, so $\left.d d^{C}=(2 \pi i)^{-1} \bar{\partial} \partial.\right)$ 
Now if $X$ is a variety over $\mathbb{R}$, then we set

$$
C_{\mathcal{D}}^{\bullet}\left(X_{/ \mathbb{R}} ; n\right):=C_{\mathcal{D}}^{\bullet}(X ; n)^{\bar{F}_{\infty}} ; \quad H_{\mathcal{D}}^{i}\left(X_{/ \mathbb{R}} ; \mathbb{R}(n)\right):=H^{i}\left(C_{\mathcal{D}}^{\bullet}\left(X_{/ \mathbb{R}} ; n\right)\right)
$$

where $\bar{F}_{\infty}$ is the de Rham involution, i.e., the composition of the involution $F_{\infty}$ on $X(\mathbb{C})$ induced by complex conjugation with the complex conjugation of coefficients.

Theorem-Construction 2.3. Let $X$ be a regular complex projective variety. Then there exists a canonical homomorphism of complexes

$$
\mathcal{P}^{\bullet}(n): \mathcal{Z}^{\bullet}(X ; n) \longrightarrow C_{\mathcal{D}}^{\bullet}(X ; n) .
$$

If $X$ is defined over $\mathbb{R}$, then the image of the map $\mathcal{P}^{\bullet}(n)$ lies in the subcomplex $C_{\mathcal{D}}^{\bullet}\left(X_{/ \mathbb{R}} ; n\right)$.

To construct this homomorphism we need to define a certain homomorphism $r_{n-1}$ (G5). In the next subsection we recall its definition and establish its basic properties. Using it we define an $(m-1)$-form $r_{m-1}(L ; H)$ canonically attached to the pair $\left(\mathbb{A}^{m} ; L\right)=\left(\mathbb{P}^{m}-H, L\right)$ and then define the homomorphism $\mathcal{P} \bullet(n)$.

4. The homomorphism $r_{m-1}$. Let $X$ be a variety over $\mathbb{C}$. Let $f_{1}, \ldots, f_{m}$ be $m$ rational functions on $X$. We attach to them the $(m-1)$-form

$$
r_{m-1}\left(f_{1}, \ldots, f_{m}\right):=
$$

$\operatorname{Alt}_{m} \sum_{j \geq 0,2 j+1 \leq 2 m+1} c_{j, m} \log \left|f_{1}\right| d \log \left|f_{2}\right| \wedge \ldots \wedge d \log \left|f_{2 j+1}\right| \wedge d i \arg f_{2 j+2} \wedge \ldots \wedge d i \arg f_{m}$.

Here $c_{j, m}:=\frac{1}{(2 j+1) !(m-2 j-1) !}$ and $\mathrm{Alt}_{m}$ is the operation of alternation:

$$
\operatorname{Alt}_{m} F\left(x_{1}, \ldots, x_{m}\right):=\sum_{\sigma \in S_{m}}(-1)^{|\sigma|} F\left(x_{\sigma(1)}, \ldots, x_{\sigma(m)}\right) .
$$

So $r_{m-1}\left(f_{1}, \ldots, f_{m}\right)$ is an $\mathbb{R}(m-1)$-valued $(m-1)$-form, and it is easy to check that

$$
d r_{m-1}\left(f_{1}, \ldots, f_{m}\right)=\pi_{m}\left(d \log f_{1} \wedge \ldots \wedge d \log f_{m}\right) .
$$

The form (15) is a part of a cocycle representing the product in real Deligne cohomology of 1-cocycles $\left(\log \left|f_{i}\right|, d \log f_{i}\right)$.

Here is yet another, a bit more general way to look at the homomorphism $r_{m-1}$. Let $\mathcal{A}^{i}(M)$ be the space of smooth $i$-forms on a real smooth manifold $M$. Consider the following map:

$$
\begin{gathered}
\omega_{m-1}: \Lambda^{m} \mathcal{A}^{0}(M) \longrightarrow \mathcal{A}^{m-1}(M) \\
\omega_{m-1}\left(\varphi_{1} \wedge \ldots \wedge \varphi_{m}\right):= \\
\frac{1}{m !} \operatorname{Alt}_{m}\left(\sum_{k=1}^{m}(-1)^{k-1} \varphi_{1} \partial \varphi_{2} \wedge \ldots \partial \varphi_{k} \wedge \bar{\partial} \varphi_{k+1} \wedge \ldots \wedge \partial \varphi_{m}\right) .
\end{gathered}
$$

For example,

$$
\omega_{0}\left(\varphi_{1}\right)=\varphi_{1} ; \quad \omega_{1}\left(\varphi_{1} \wedge \varphi_{2}\right)=\frac{1}{2}\left(\varphi_{1} \partial \varphi_{2}-\varphi_{2} \partial \varphi_{1}-\varphi_{1} \bar{\partial} \varphi_{2}+\varphi_{2} \bar{\partial} \varphi_{1}\right)
$$


Then one easily checks that

$$
\begin{gathered}
d \omega_{m-1}\left(\varphi_{1} \wedge \ldots \wedge \varphi_{m}\right)=\partial \varphi_{1} \wedge \ldots \wedge \partial \varphi_{m}+(-1)^{m} \bar{\partial} \varphi_{1} \wedge \ldots \wedge \bar{\partial} \varphi_{m} \\
+\sum_{i=1}^{m}(-1)^{i} \bar{\partial} \partial \varphi_{i} \wedge \omega_{m-2}\left(\varphi_{1} \wedge \ldots \wedge \widehat{\varphi}_{i} \wedge \ldots \wedge \varphi_{m}\right) .
\end{gathered}
$$

Now let $f_{i}$ be rational functions on a complex algebraic variety $X$. Set $M:=$ $X^{0}(\mathbb{C})$, where $X^{0}$ is the open part of $X$ where the functions $f_{i}$ are regular. Then $\varphi_{i}:=\log \left|f_{i}\right|$ are smooth functions on $M$, and we have an identity

$$
\omega_{m-1}\left(\log \left|f_{1}\right| \wedge \ldots \wedge \log \left|f_{m}\right|\right)=r_{m-1}\left(f_{1} \wedge \ldots \wedge f_{m}\right) .
$$

Observe that $\bar{\partial} \partial \log |f|=0$ on $X^{0}(\mathbb{C})$. Therefore the second term in the formula (18) is zero, and so this formula is consistent with the one in (16). Notice however that if we understood $\bar{\partial} \partial \log |f|$ as a distribution on $X(\mathbb{C})$, then by the PoincaréLelong formula one has

$$
2 \bar{\partial} \partial \log |f|=2 \pi i \delta(f)
$$

Our next goal is to interpret the form $r_{m-1}\left(f_{1} \wedge \ldots \wedge f_{m}\right)$ as a distribution on $X(\mathbb{C})$ and calculate the differential of this distribution, taking into account formula (19).

5. The distribution $r_{m-1}\left(f_{1} \wedge \ldots \wedge f_{m}\right)$. Recall (see for instance $[\underline{S}]$ ) that for a subvariety $Y$ of a smooth complex variety $X$ we define the $\delta$-distribution $\delta_{Y}$ by setting

$$
\left\langle\delta_{Y}, \omega\right\rangle:=\int_{Y^{0}(\mathbb{C})} \omega
$$

where $Y^{0}$ is the nonsingular part of $Y$.

Theorem 2.4. Let $Y$ be an arbitrary irreducible subvariety of a smooth complex variety $X$ and $f_{1}, \ldots, f_{m} \in \mathbb{C}^{*}(Y)$. Then for any smooth differential form $\omega$ with compact support on $X(\mathbb{C})$ the following integral is convergent:

$$
\int_{Y^{0}(\mathbb{C})} r_{m-1}\left(f_{1}, \ldots, f_{m}\right) \wedge i_{Y}^{0} \omega .
$$

Here $Y^{0}$ is the nonsingular part of $Y$ and $i_{Y}^{0} \omega$ is the restriction of the form $\omega$ to $Y^{0}(\mathbb{C})$. Thus the form $r_{m-1}\left(f_{1}, \ldots, f_{m}\right)$ defines a distribution $r_{m-1}\left(f_{1}, \ldots, f_{m}\right) \delta_{Y}$ on $X(\mathbb{C})$ given by

$$
\left\langle r_{m-1}\left(f_{1}, \ldots, f_{m}\right) \delta_{Y}, \omega\right\rangle:=\int_{Y^{0}(\mathbb{C})} r_{m-1}\left(f_{1}, \ldots, f_{m}\right) \wedge i_{Y}^{0} \omega .
$$

It provides a group homomorphism

$$
r_{m-1}: \Lambda^{m} \mathbb{C}(Y)^{*} \longrightarrow \mathcal{D}_{X(\mathbb{C})}^{m-1}(m-1) .
$$

Proof. We need the following lemma.

Lemma 2.5. Let $Y$ be a smooth complex projective variety. Then for any nonzero rational functions $f_{1}, \ldots, f_{m}$ on $Y$ and for any smooth form $\omega$ with compact support on $Y(\mathbb{C})$ the integral

$$
\int_{Y(\mathbb{C})} r_{m-1}\left(f_{1}, \ldots, f_{m}\right) \wedge \omega
$$

is convergent. So the form $r_{m-1}\left(f_{1}, \ldots, f_{m}\right)$ defines a distribution on $Y(\mathbb{C})$. 
Basic example. The integral $\int_{\mathbb{C}} \log |z| d \log (z-a) \wedge d \log \overline{(z-b)}$ is divergent at infinity, where all the functions $z, z-a, z-b$ have a simple pole, since $\int_{\mathbb{C}} \log |z| \frac{d z \wedge d \bar{z}}{|z|^{2}}$ is divergent (both near zero and infinity). However,

$$
\begin{aligned}
& 4 \cdot \int_{\mathbb{C}} \log |z| d \log |z-a| \wedge d \log |z-b| \\
& \quad=\int_{\mathbb{C}} \log |z|(d \log (z-a) \wedge d \log \overline{(z-b)}+d \log \overline{(z-a)} \wedge d \log (z-b)) .
\end{aligned}
$$

is convergent: the divergent parts cancel each other. In $r_{m-1}\left(f_{1}, \ldots, f_{m}\right)$ such divergences cancel because of the multiplicativity and skew-symmetry of $r_{m-1}$.

Proof of Lemma 2.5. Resolving singularities we reduce the statement of the lemma to the case when the $\operatorname{divisors} \operatorname{div} f_{i}$ have normal crossing. Using the fact that $r_{m-1}$ is a homomorphism to differential forms we may suppose that these divisors are different. Our statement is local, so we can assume that in local coordinates $z_{1}, \ldots, z_{m}$ one has $f_{1}=z_{1}, \ldots, f_{k}=z_{k}$ and $\operatorname{div} f_{j}$ for $j>k$ does not intersect the origin. After this the statement of the lemma is obvious: each term in (15) defines a distribution near the origin. For instance, the worst possible singularities have the term $\log \left|z_{1}\right| d \log \left|z_{2}\right| \wedge \ldots \wedge d \log \left|z_{k}\right| \wedge \omega$ where $\omega$ is smooth near the origin. It is clearly integrable with a smooth test form. The lemma is proved.

Remark. In particular, if $\operatorname{dim}_{\mathbb{C}} X=n$, the integral

$$
\int_{X(\mathbb{C})} r_{2 n}\left(f_{1}, \ldots, f_{2 n+1}\right)
$$

is convergent.

Below we use the following form of the resolution of singularities theorem. Recall that the proper preimage $\widetilde{Y}$ is the closure in $\widetilde{X}$ of the preimage of the generic part of $Y$.

Theorem 2.6. Let $Y$ be an arbitrary subvariety of a regular variety $X$ over a characteristic zero field, and $Z$ a divisor of $Y$. Then there exists a sequence of blowups $\widetilde{X} \longrightarrow X_{1} \longrightarrow \ldots \longrightarrow X$ providing a projection $\pi: \widetilde{X} \rightarrow X$ such that the proper preimage $\widetilde{Y}$ of $Y$ is nonsingular, $\widetilde{Z}:=p^{*} Z$, where $p=\left.\pi\right|_{\widetilde{Y}}: \widetilde{Y} \rightarrow Y$ is a normal crossing divisor in $\widetilde{Y}$ and the restriction of $\pi$ to the nonsingular part of $\widetilde{Y}-\widetilde{Z}$ is an isomorphism.

Proof of Theorem 2.4. By Theorem[2.6]there exists a sequence of blowups providing a projection $\pi: \widetilde{X} \longrightarrow X$ such that the proper preimage $\widetilde{Y}$ of $Y \subset X$ is smooth. By the above lemma the integral

$$
\int_{\tilde{Y}(\mathbb{C})} r_{m-1}\left(\pi^{*} f_{1}, \ldots, \pi^{*} f_{m}\right) \wedge \pi^{*} \omega
$$

is convergent. Therefore the similar integral over any Zariski dense subset of $\widetilde{Y}(\mathbb{C})$ is also convergent and coincides with (22). Since $\pi$ is an isomorphism on the nonsingular part of $\widetilde{Y}-\widetilde{Z}$, we are done. Theorem 2.4 is proved.

Below we employ the notation $r_{m-1}\left(f_{1} \wedge \ldots \wedge f_{m}\right)$ for the distribution given by (20). 
6. Differential of the distribution $r_{n-1}\left(f_{1} \wedge \ldots \wedge f_{n}\right)$. Let $X$ be a normal variety. Then there is the residue homomorphism

$$
\operatorname{Res}: \Lambda^{n} \mathbb{C}(X)^{*} \longrightarrow \bigoplus_{Y \subset X^{(1)}} \Lambda^{n-1} \mathbb{C}(Y)^{*},
$$

where the sum is over all irreducible divisors of $X$.

Here is its definition. Let $K$ be a field with a discrete valuation $v$ and the residue field $k_{v}$. The group of units $U$ has a natural homomorphism $U \longrightarrow k_{v}^{*}, u \mapsto \bar{u}$. An element $\pi \in K^{*}$ is prime if $\operatorname{ord}_{v} \pi=1$. There is a homomorphism $\operatorname{res}_{v}: \Lambda^{n} K^{*} \longrightarrow$ $\Lambda^{n-1} k_{v}^{*}$ uniquely defined by the properties $\left(u_{i} \in U\right)$ :

$$
\operatorname{res}_{v}\left(\pi \wedge u_{1} \wedge \cdots \wedge u_{n-1}\right)=\bar{u}_{1} \wedge \cdots \wedge \bar{u}_{n-1} \quad \text { and } \quad \operatorname{res}_{v}\left(u_{1} \wedge \cdots \wedge u_{n}\right)=0 .
$$

It does not depend on the choice of $\pi$.

Observe that if $X$ is normal, then the local ring of any irreducible divisor of $X$ is a discrete valuation ring, so we can apply the above construction. We set Res $:=\sum \operatorname{res}_{v}$ where the sum is over all valuations of the field $\mathbb{C}(X)$ corresponding to the codimension one points of $X$.

Remark. If for any $i$ the restrictions of the functions $f_{j}$ for $j \neq i$ to the generic points of all irreducible components of the $\operatorname{divisor} \operatorname{div} f_{i}$ are nonzero, then

$$
\operatorname{Res}\left(f_{1} \wedge \ldots \wedge f_{n}\right)=\sum_{Y \in X^{(1)}} \sum_{i=1}^{n}(-1)^{i-1} v_{Y}\left(f_{i}\right) \cdot f_{\left.1\right|_{Y}} \wedge \ldots \wedge \widehat{f}_{i_{Y}} \wedge \ldots \wedge f_{\left.n\right|_{Y}}
$$

where $v_{Y}(f)$ is the order of zero of $f$ at the generic point of $Y$.

Let $f_{1} \wedge \ldots \wedge f_{n} \in \Lambda^{n} \mathbb{C}(Y)^{*}$ where $Y$ is a subvariety of a regular complex variety $X$. We define a distribution

$$
\left(r_{n-2} \circ \operatorname{Res}\right)\left(f_{1} \wedge \ldots \wedge f_{n}\right)
$$

on $X(\mathbb{C})$ as follows.

(i) If $Y$ is a normal subvariety, then we have defined the residue map Res on $\Lambda^{n} \mathbb{C}(Y)^{*}$. So we define (24) as

$$
\sum_{Z \in Y^{(1)}} r_{n-2} \operatorname{res}_{Z}\left(f_{1} \wedge \ldots \wedge f_{n}\right) \delta_{Y}
$$

i.e., for any smooth form $\omega$ on $X(\mathbb{C})$,

$$
\left\langle\left(r_{n-2} \circ \operatorname{Res}\right)\left(f_{1} \wedge \ldots \wedge f_{n}\right), \omega\right\rangle:=\sum_{Z} \int_{Z^{0}(\mathbb{C})} r_{n-2} \operatorname{res}_{Z}\left(f_{1} \wedge \ldots \wedge f_{n}\right) \wedge \omega .
$$

(ii) For an arbitrary $Y$ we take the normalization $\pi: Y^{\nu} \rightarrow Y$ and define (24) as

$$
\left(r_{n-2} \circ \operatorname{Res}\right)\left(f_{1} \wedge \ldots \wedge f_{n}\right):=\pi_{*}\left(\left(r_{n-2} \circ \operatorname{Res}\right)\left(\pi^{*} f_{1} \wedge \ldots \wedge \pi^{*} f_{n}\right)\right) .
$$

Lemma 2.7. Let $Y$ be a subvariety of a regular complex variety $X, f_{1} \wedge \ldots \wedge f_{n} \in$ $\Lambda^{n} \mathbb{C}(Y)^{*}$, and $Z:=\bigcup_{i} \operatorname{div} f_{i}$. Let $\pi: \widetilde{X} \rightarrow X$ be a blowup of $X$ as in Theorem [2.6. Then

$$
\pi_{*}\left(\left(r_{n-2} \circ \operatorname{Res}\right)\left(\pi^{*} f_{1} \wedge \ldots \wedge \pi^{*} f_{n}\right)\right)=\left(r_{n-2} \circ \operatorname{Res}\right)\left(f_{1} \wedge \ldots \wedge f_{n}\right) .
$$

Proof. Thanks to the definition (ii) of distribution (24) for nonnormal varieties we may assume without loss of generality that $Y$ is normal.

Let $Z=\bigcup_{i \in I} Z_{i}$ be the decomposition of the divisor $Z$ into irreducible components parametrised by a set $I$. By the very definition the right-hand side of (25) 
is a sum over $i \in I$ of distributions $\psi_{i}=r_{n-2}\left(F_{i}\right)$ on $Z_{i}$ corresponding to certain elements $F_{i} \in \Lambda^{n-2} \mathbb{C}\left(Z_{i}\right)^{*}$.

Let $\widetilde{Z}=\bigcup_{j \in J} \widetilde{Z}_{j}$ be the decomposition of $\widetilde{Z}$ into irreducible components parametrized by a set $J$. The left-hand side of (25) is a sum over $j \in J$ of distributions $\widetilde{\psi}_{j}=r_{n-2}\left(\widetilde{F}_{j}\right)$ corresponding to certain elements $\widetilde{F}_{j} \in \Lambda^{n-2} \mathbb{C}\left(\widetilde{Z}_{j}\right)^{*}$. One has $I \subset J$ since the proper preimage $\widetilde{Z}_{i}$ of $Z_{i}$ is an irreducible component of $\widetilde{Z}$.

The lemma follows from the following two claims:

$$
\pi_{*}\left(\widetilde{\psi}_{i}\right)=\psi_{i}, \quad i \in I ; \quad \pi_{*}\left(\widetilde{\psi}_{j}\right)=0, \quad j \in J-I .
$$

The first one is obvious since both distributions $\widetilde{\psi}_{i}$ and $\psi_{i}$ are defined by their restriction to any nonsingular Zariski dense open part of the corresponding divisor, and $\pi$, being restricted to such a sufficiently small part of $\widetilde{Z}_{i}$, is an isomorphism. Let us prove the second claim. Observe that for $j \in J-I$ the subvariety $\pi\left(\widetilde{Z}_{j}\right)$ is of codimension 2 in $Y$, and the restriction of $\pi$ to a Zariski open part of $\widetilde{Z}_{j}$ is a fibration with fibers of positive dimension. We need to show that for any smooth form $\omega$ on $X(\mathbb{C})$,

$$
\int_{\widetilde{Z}_{j}(\mathbb{C})} \widetilde{\psi}_{j} \wedge \pi^{*} \omega:=\int_{\widetilde{Z}_{j}^{\prime}(\mathbb{C})} \widetilde{\psi}_{j} \wedge \pi^{*} \omega=0
$$

where $Z_{j}^{\prime}$ is a (sufficiently small) nonsingular Zariski dense open part of $Z_{j}$. Observe that $\widetilde{\psi}_{j} \wedge \pi^{*} \omega$ is a smooth form on $\widetilde{Z}_{j}^{\prime}(\mathbb{C})$. For any vector field $v$ on $\widetilde{Z}_{j}^{\prime}$ tangent to the fibers of $\pi$ we have $i_{v} \pi^{*} \omega=0$. So it suffices to show that $i_{v} \widetilde{\psi}_{j}=0$. Since the statement is local, we can choose a local equation of an open part of $\widetilde{Z}_{j}^{\prime}$ in the form $\pi^{*} z=0$. Using it as a local parameter at the definition of $\operatorname{res}_{\pi^{*} z=0}$, we see that $\operatorname{res}_{\pi^{*} z=0}\left(\pi^{*} f_{1} \wedge \ldots \wedge \pi^{*} f_{n}\right)$ is lifted from $Y$, and hence so is

$$
\widetilde{\psi}_{j}=r_{n-2} \operatorname{res}_{\pi^{*} z=0}\left(\pi^{*} f_{1} \wedge \ldots \wedge \pi^{*} f_{n}\right) .
$$

Thus $i_{v} \widetilde{\psi}_{j}=0$. The lemma is proved.

Proposition 2.8. Let $Y$ be an arbitrary subvariety of a regular complex variety $X$ and $f_{1} \wedge \ldots \wedge f_{n} \in \Lambda^{n} \mathbb{C}(Y)^{*}$. Then

$$
\begin{gathered}
d r_{n-1}\left(f_{1} \wedge \ldots \wedge f_{n}\right) \\
=\pi_{n}\left(d \log f_{1} \wedge \ldots \wedge d \log f_{n}\right)+2 \pi i \cdot\left(r_{n-2} \circ \operatorname{Res}\right)\left(f_{1} \wedge \ldots \wedge f_{n}\right) .
\end{gathered}
$$

Proof. Let us resolve singularities as in Lemma 2.7 Since $\pi$ is a birational isomorphism, and the distribution $r_{n-1}\left(f_{1} \wedge \ldots \wedge f_{n}\right)$ is determined by its restriction to the generic point, one has

$$
\pi_{*} r_{n-1}\left(\pi^{*} f_{1} \wedge \ldots \wedge \pi^{*} f_{n}\right)=r_{n-1}\left(f_{1} \wedge \ldots \wedge f_{n}\right) .
$$

So this and Lemma 2.7 imply that we may assume that $\bigcup_{i} \operatorname{div} f_{i}$ is a normal crossing divisor. The proposition follows immediately from the Poincaré-Lelong formula (19)

$$
d(d i \arg f)=2 \pi i \delta(f):=2 \pi i \delta_{\operatorname{div}(f)} .
$$

The Proposition 2.8 is proved.

Remark. To prove the Poincaré-Lelong formula one may resolve the singularities and argue just as in the proof of Lemma[2.7] that it is sufficient to prove this formula 
on a blowup. When the divisor of $f$ is a normal crossing divisor the formula follows from

$$
d(d i \arg z)=2 \pi i \delta(z):=2 \pi i\left(\delta_{0}-\delta_{\infty}\right) .
$$

7. The distribution $r_{m-1}(L ; H)$. Let $\Omega_{L}$ be the canonical $m$-form in $\mathbb{P}^{m}-L$ with logarithmic singularities at $L$. It represents a generator of $H_{\mathrm{DR}}^{m}\left(\mathbb{P}^{m}-L\right)$ defined over $\mathbb{Z}$. Let us give its coordinate description. Choose homogeneous coordinates $\left(z_{0}: \ldots: z_{m}\right)$ in $\mathbb{P}^{m}$ such that $L_{i}$ is given by the equation $\left\{z_{i}=0\right\}$. Then

$$
\Omega_{L}=d \log z_{1} / z_{0} \wedge \ldots \wedge d \log z_{m} / z_{0} .
$$

The form $\Omega_{L}$ has periods in $\mathbb{Z}(m)$. So $\pi_{m}\left(\Omega_{L}\right)$ is exact. However there is no canonical choice of a primitive $(m-1)$-form for it: the group $\left(\mathbb{C}^{*}\right)^{m}$ acting on $\mathbb{C P}^{m}-L$ leaves the form invariant and acts nontrivially on the primitives. But if we consider a simplex $L$ in the affine complex space $\mathbb{A}^{m}$ (or, what is the same, choose an additional hyperplane $H$ in $\mathbb{C P}^{m}$, which should be thought of as the infinite hyperplane), then there is a canonical primitive.

Choose a coordinate system $\left(z_{0}: \ldots: z_{m}\right)$ in $\mathbb{P}^{m}$ as above such that $H$ is given by $\left\{\sum_{i=1}^{m} z_{i}=z_{0}\right\}$. Set

$$
r_{m-1}(L ; H):=r_{m-1}\left(z_{1} / z_{0} \wedge \ldots \wedge z_{m} / z_{0}\right) .
$$

Here is a more invariant definition (see Figure [5). Choose one of the faces of the simplex $L$, say $L_{0}$. Consider the simplex $\left(H, L_{1}, \ldots, L_{m}\right)$. Let $f_{i}$ be the rational function on $\mathbb{C P}^{m}$ such that $\left(f_{i}\right)=L_{i}-L_{0}$ is normalized by $f_{i}\left(l_{i}\right)=1$, where $l_{i}$ is the vertex of the simplex $\left(H, L_{1}, \ldots, L_{m}\right)$ opposite to the face $L_{i}$. Then $f_{i}=\frac{z_{i}}{z_{0}}$ and

$$
r_{m-1}(L ; H):=r_{m-1}\left(f_{1} \wedge \ldots \wedge f_{m}\right) .
$$

This form is skewsymmetric with respect to the permutation of the hyperplane faces of the simplex $L$. One has

$$
d r_{m-1}(L ; H)=\pi_{m}\left(\Omega_{L}\right) \text { in } \mathbb{C P}^{m}-L .
$$

So a choice of an "infinite" hyperplane $H \subset \mathbb{C P}^{m}$ provides the form $r_{m-1}(L ; H)$.

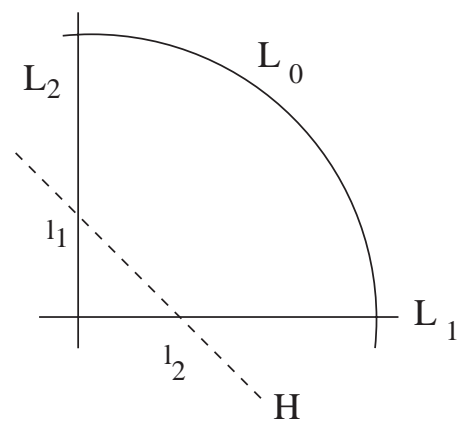

FiguRE 5. A simplex $L$ and an infinite hyperplane $H$

Example. If $m=1$, then

$$
\begin{aligned}
\mathbb{A}^{1}=\mathbb{P}^{1}-\{1\}, \quad L= & \{0\} \cup\{\infty\}, \quad \Omega_{L}=d \log z, \quad \pi_{1}(d \log z)=d \log |z|, \\
& r_{0}(\{0\} \cup\{\infty\} ;\{1\})=\log |z| .
\end{aligned}
$$


The $(n-1)$-form $r_{n-1}(L ; H)$ provides an $(n-1)$-distribution on $\mathbb{C P}^{n}$. Recall the simplex $\widehat{L}_{i}$ which is cut out by $L$ in the hyperplane $L_{i}$, and put $H_{i}:=L_{i} \cap H$. Consider the $(n-2)$-form $r_{n-2}\left(\widehat{L}_{i} ; H_{i}\right)$ on the hyperplane $L_{i}$ as an $n$-distribution in $\mathbb{C P}^{n}$. We denote it as $r_{n-2}\left(\widehat{L}_{i} ; H_{i}\right) \cdot \delta_{L_{i}}$.

Corollary 2.9. One has

$$
d r_{n-1}(L ; H)=\pi_{n}\left(\Omega_{L}\right)+2 \pi i \cdot \sum_{i=0}^{n}(-1)^{i} r_{n-2}\left(\widehat{L}_{i} ; H_{i}\right) \delta_{L_{i}} .
$$

Proof. This follows immediately from Proposition 2.8 .

8. A coordinate-free description of the form $r_{m-1}(L ; H)$. Let $V_{m}$ be an $m$-dimensional vector space over a field $F$. Choose a volume form $\operatorname{vol}_{m} \in \operatorname{det} V_{m}^{*}$. Set $\Delta\left(v_{1}, \ldots, v_{m}\right):=\left\langle\operatorname{vol}_{m}, v_{1} \wedge \ldots \wedge v_{m}\right\rangle \in F^{*}$.

Lemma 2.10. For a configuration $\left(l_{0}, \ldots, l_{m}\right)$ of $m+1$ vectors in generic position,

$$
f_{m}\left(l_{0}, \ldots, l_{m}\right):=\sum_{i=0}^{m}(-1)^{i} \Lambda_{j \neq i} \Delta\left(l_{0}, \ldots, \widehat{l}_{j}, \ldots, l_{i}, \ldots, l_{m}\right) \in \Lambda^{m} F^{*}
$$

does not depend on the choice of the volume form $\mathrm{vol}_{m}$.

Proof. See the proof of Lemma 3.1 in G3. 6]

For a point $z \in \mathbb{A}^{m}-L$, let $l_{i}(z)$ be the vector from $z$ to the vertex $l_{i}$; see Figure

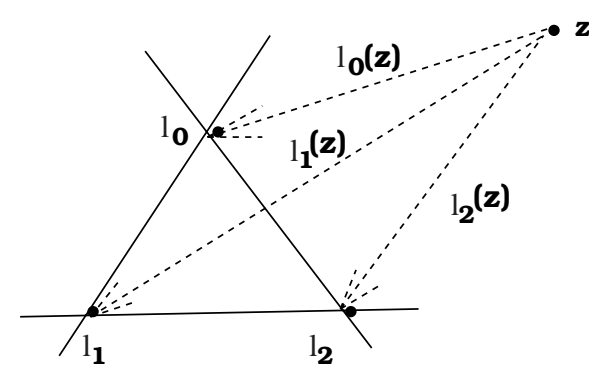

Figure 6. The vectors $l_{i}(z)$

We get a canonical element

$$
f_{m}\left(l_{0}(z), \ldots, l_{m}(z)\right) \in \Lambda^{m} \mathbb{Q}\left(\mathbb{A}^{m}-L\right)^{*} .
$$

If $F=\mathbb{C}$, applying the homomorphism $r_{m}$ to this element we get a canonical $(m-1)$-form in $\mathbb{C P}^{m}-L$. It coincides with $r_{m-1}(L ; H)$.

Example. If $m=1$ and $\left(L_{0}, L_{1}, H\right)=(0, \infty, 1)$, then $t=\frac{z}{z-1}$ is an affine coordinate on $\mathbb{P}^{1}-\{1\}$ and

$$
l_{0}(t)=\frac{z}{z-1}, l_{1}(t)=\frac{1}{z-1}, \quad \text { so } \quad f_{1}\left(l_{0}(t), l_{1}(t)\right)=\frac{l_{0}(t)}{l_{1}(t)}=z .
$$


Remark. The map $z \in \mathbb{A}^{m}-L \longmapsto f_{m}\left(l_{0}(z), \ldots, l_{m}(z)\right)$ provides an isomorphism $F_{m}: C H^{m}(\operatorname{Spec}(F), 0) \longrightarrow K_{m}^{M}(F)$; see [NS] where the isomorphism $F_{m}$ was presented in a bit different way.

9. The main construction. We have to construct a morphism of complexes

$$
\begin{aligned}
& \ldots \quad \longrightarrow \mathcal{Z}^{1}(X ; n) \quad \longrightarrow \quad \ldots \quad \longrightarrow \mathcal{Z}^{2 n-1}(X ; n) \quad \longrightarrow \mathcal{Z}^{2 n}(X ; n) \\
& \downarrow \mathcal{P}^{1}(n) \quad \ldots \quad \downarrow \mathcal{P}^{2 n-1}(n) \quad \downarrow \mathcal{P}^{2 n}(n) \\
& 0 \quad \longrightarrow \mathcal{D}_{\mathbb{R}}^{0,0}(n-1) \quad \longrightarrow \quad \ldots \quad \longrightarrow \mathcal{D}_{\mathbb{R}}^{n-1, n-1}(n-1) \stackrel{2 \bar{\partial} \partial}{\longrightarrow} \mathcal{D}_{\mathbb{R}}^{n, n}(n)
\end{aligned}
$$

Let $Y \in \mathcal{Z}^{2 n}(X ; n)$ be a codimension $n$ cycle in $X$. By definition,

$$
\mathcal{P}^{2 n}(n)(Y):=(2 \pi i)^{n} \delta_{Y} \quad \text { where } \quad\left\langle\delta_{Y}, \omega\right\rangle:=\int_{Y(\mathbb{C})} \omega .
$$

Let us construct homomorphisms

$$
\mathcal{P}^{2 n-i}(n): \mathcal{Z}^{2 n-i}(X ; n) \longrightarrow \mathcal{D}_{X(\mathbb{C})}^{2 n-i-1}(n-1), \quad i>0 .
$$

Denote by $\pi_{\mathbb{A}^{i}}\left(\right.$ resp. $\left.\pi_{X}\right)$ the projection of $X \times \mathbb{A}^{i}$ to $\mathbb{A}^{i}$ (resp. $X$ ), and by $\bar{\pi}_{\mathbb{A}^{i}}$ (resp. $\bar{\pi}_{X}$ ) the projection of $X \times \mathbb{C P}^{i}$ to $\mathbb{C P}^{i}$ (resp. $X$ ).

Recall the element

$$
\frac{z_{1}}{z_{0}} \wedge \ldots \wedge \frac{z_{i}}{z_{0}} \in \Lambda^{i} \mathbb{C}\left(\mathbb{A}^{i}\right)^{*}
$$

defining the form $r_{i-1}(L ; H)$; see (27). Let

$$
g_{1} \wedge \ldots \wedge g_{i} \in \Lambda^{i} \mathbb{C}(Y)^{*}
$$

be the restriction to $Y$ of the inverse image of element (30) by the projection $\pi_{\AA^{i}}^{*}$. The element (31) provides, by Theorem 2.4, a distribution on $X(\mathbb{C}) \times \mathbb{C P}^{i}$. Pushing this distribution down by $(2 \pi i)^{n-i} \cdot \pi_{\bar{X}}$ we get the distribution $\mathcal{P}^{2 n-i}(n)(Y)$.

\section{Definition 2.11.}

$$
\begin{aligned}
\mathcal{P}^{2 n-i}(n)(Y) & :=(2 \pi i)^{n-i} \cdot \pi_{\bar{X} *} r_{i-1}\left(g_{1} \wedge \ldots \wedge g_{i}\right) \\
& :=(2 \pi i)^{n-i} \cdot \pi_{\bar{X} *} r_{i-1}\left(i_{Y}^{*} \pi_{\mathbb{A}^{i}}^{*}\left(\frac{z_{1}}{z_{0}} \wedge \ldots \wedge \frac{z_{i}}{z_{0}}\right)\right) .
\end{aligned}
$$

Here $i_{Y}: Y \hookrightarrow X \times \mathbb{P}^{i}$.

Remark. This definition works if and only if the cycle $Y$ has proper intersection with all codimension one faces of $X \times L$. Indeed, if $Y$ does not have proper intersection with one of the faces, then the equation of this face restricts to zero to $Y$, and so (31) does not make sense. As soon as all equations of the codimension one faces restrict to nonzero functions on $Y$, (31) makes sense, and we can apply Theorem 2.4 .

Remark. We just proved that the product of distributions $\delta_{Y} \wedge \pi_{\mathbb{A}}^{*} r_{i-1}(L ; H)$ makes sense and

$$
\mathcal{P}^{2 n-i}(n)(Y)=(2 \pi i)^{n-i} \pi_{X *}\left(\delta_{Y} \wedge \pi_{\mathbb{A}}^{*} r_{i-1}(L ; H)\right) .
$$


It is handy to rewrite Definition 2.11 more explicitly as an integral over $Y(\mathbb{C})$. Namely, let $\omega$ be a smooth form on $X(\mathbb{C})$ and $Y \in \mathcal{Z}^{2 n-i}(X ; n)$. Then

$$
\begin{gathered}
\left\langle\mathcal{P}^{2 n-i}(n)(Y), \omega\right\rangle=(2 \pi i)^{n-i} \int_{Y(\mathbb{C})} \pi_{\mathbb{A}}^{*} r_{i-1}(L ; H) \wedge \pi_{X}^{*} \omega \\
=(2 \pi i)^{n-i} \int_{Y^{0}(\mathbb{C})} r_{i-1}\left(g_{1} \wedge \ldots \wedge g_{i}\right) \wedge i_{Y(\mathbb{C})}^{*} \pi_{X}^{*} \omega
\end{gathered}
$$

where $Y^{0}$ is the nonsingular part of $Y$

Since the form $r_{i-1}(L ; H)$ is $\mathbb{R}(i-1)$-valued, for $i>0$ the distribution $\mathcal{P}^{2 n-i}(n)(Y)$ takes values in $\mathbb{R}(n-1)$. Furthermore, $\mathcal{P}^{2 n}(n)(Y)$ is obviously an $\mathbb{R}(n)$-valued distribution.

Let us show that for $i>0$ the distribution $\mathcal{P}^{2 n-i}(n)(Y)$ lies precisely in the left bottom $(n-1) \times(n-1)$ square of the Dolbeault bicomplex. The integral (32) is nonzero only if $\pi_{X}^{*} \omega \wedge \pi_{\mathbb{A}}^{*} r_{i-1}(L ; H)$ is of type

$$
(\operatorname{dim} Y, \operatorname{dim} Y)=(\operatorname{dim} X+i-n, \operatorname{dim} X+i-n) .
$$

Since $r_{i-1}(L ; H)$ is an $(i-1)$-form we see that the integral vanishes if $\omega$ is a form of type $(p, q)$ where $p$ or $q$ is smaller than $\operatorname{dim} X+1-n$. This just means that the distribution lies in the left bottom $(n-1) \times(n-1)$ square of the Dolbeault bicomplex. The proposition is proved.

Therefore we have constructed the maps $\mathcal{P}^{i}(n)$.

Theorem 2.12. $\mathcal{P}^{\bullet}(n)$ is a homomorphism of complexes.

Proof. One has

$$
\left\langle d \mathcal{P}^{2 n-i}(n)(Y), \omega\right\rangle=\int_{\bar{Y}(\mathbb{C})} d r_{i-1}\left(g_{1} \wedge \ldots \wedge g_{i}\right) \wedge i_{\bar{Y}}^{*} \pi_{X}^{*} \omega
$$

where $g_{1} \wedge \ldots \wedge g_{i}$ is as in (31). We use Proposition 2.8 to calculate $d r_{i-1}\left(g_{1} \wedge \ldots \wedge g_{i}\right)$.

To handle the first term in (26) observe that by the very definition of the complex $\mathcal{C}_{\mathcal{D}}(X ; n)$ we need to investigate integral (34) only for smooth forms $\omega$ of type $(p, q)$ where $|p-q| \leq i-1$. Since $\pi_{i}\left(\Omega_{L}\right)=\Omega_{L} \pm \bar{\Omega}_{L}$ is a sum of forms of type $(i, 0)$ and $(0, i)$ the form $\pi_{\mathbb{A}}^{*} \pi_{i}\left(\Omega_{L}\right) \wedge \pi_{X}^{*} \omega$ cannot be of type $(k, k)$. Therefore only the second term in (26) contributes.

Since $r_{0}(f)=\log |f|$, the commutativity of the last square follows from the Poincaré-Lelong formula $2 \bar{\partial} \partial \log |f|=2 \pi i \cdot \delta_{\operatorname{div}(f)}$.

Commutativity of the $i$-th square of the diagram, $i>1$, counting from the right. If $\bar{Y}$ is normal, this follows from Proposition 2.8 Indeed, since $Y$ meets the codimension two faces properly, all equations of the codimension one faces of $X \times L$ but $z_{j}$ have nonzero restriction to the generic point of $\operatorname{div} z_{j}$. Therefore we may use formula (23) to calculate Res, and then the claim is obvious.

In the case when $\bar{Y}$ is not normal we face the following subtle problem.

Calculating $d r_{i-1}\left(g_{1} \wedge \ldots \wedge g_{i}\right)$ and hence $d \circ \mathcal{P}^{2 n-i}(n)(Y)$ when $Y$ is not normal we need to take $\operatorname{Res}_{\widetilde{Z}}\left(\widetilde{g}_{1} \wedge \ldots \wedge \widetilde{g}_{i}\right)$ for all irreducible divisors $\widetilde{Z}$ in the normalization $\pi: \tilde{Y} \rightarrow Y$, where $\widetilde{g}:=\pi^{*} g$, and then take

$$
\sum_{\widetilde{Z}} \pi_{*} r_{i-2} \circ \operatorname{Res}_{\widetilde{Z}}\left(\widetilde{g}_{1} \wedge \ldots \wedge \widetilde{g}_{i}\right) .
$$


Computation of $\mathcal{P}^{2 n-i+1}(n)(Z) \circ d$ does not involve the normalization of $Y$ : we intersect $Y$ with all codimension one faces of $X \times L$. So we need to compute (35) using the intersection data of $Y$ and $X \times L$.

To handle this we use the condition that $Y$ meets the codimension two faces of the simplex $X \times L$ properly. This implies that

$$
\operatorname{Res}_{\widetilde{Z}}\left(\widetilde{g}_{1} \wedge \ldots \wedge \widetilde{g}_{i}\right)=\pi^{*} G_{i-1} ; \quad G_{i-1} \in \Lambda^{i-1} \mathbb{C}(\pi(\widetilde{Z}))^{*} .
$$

Indeed, since all equations of the codimension one faces of $X \times L$ but $z_{j}$ have nonzero restriction to the generic point of $\operatorname{div} z_{j}$, the wedge product of these restrictions can be taken as $G_{i-1}$.

Given (36) the statement is obvious since

$$
\pi_{*} r_{i-2} \pi^{*} G_{i-1}=[\mathbb{C}(\widetilde{Z}): \mathbb{C}(\pi(\widetilde{Z}))] \cdot r_{i-2}\left(G_{i-1}\right) .
$$

Indeed, recall (see $[\mathbf{F}$, page 9 ) that if $\tilde{Y} \rightarrow Y$ is the normalization of $Y$ and $g \in \mathbb{C}(Y)^{*}=\mathbb{C}(\widetilde{Y})^{*}$, then

$$
\operatorname{ord}_{Z}(g)=\sum_{\widetilde{Z}} \operatorname{ord}_{\widetilde{Z}}(g)[\mathbb{C}(\widetilde{Z}): \mathbb{C}(Z)]
$$

where the sum is over all irreducible divisors projecting onto $Z$.

Theorem 2.12 is proved. Therefore we have finished the proof of TheoremConstruction 2.3.

10. The higher Arakelov Chow groups. Let $X$ be a regular complex variety. Denote by $\widetilde{C}_{\mathcal{D}}^{\bullet}(n)$ the quotient of the complex $C_{\mathcal{D}}^{\bullet}(n)$ along the subgroup $\mathcal{A}_{c l}^{n, n}(n) \subset \mathcal{D}_{c l}^{n, n}(n)$ of a closed smooth form of type $(n, n)$ with values in $\mathbb{R}(n)$.

Consider the cone of the homomorphism $\mathcal{P}^{\bullet}(n)$ shifted by -1 :

$$
\widehat{\mathcal{Z}}^{\bullet}(X ; n):=\operatorname{Cone}\left(\mathcal{Z}^{\bullet}(X ; n) \longrightarrow \widetilde{C}_{\mathcal{D}}^{\bullet}(X(\mathbb{C}) ; n)\right)[-1] .
$$

Definition 2.13. The higher Arakelov Chow groups are

$$
\widehat{C H}^{n}(X ; i):=H^{2 n-i}(\widehat{\mathcal{Z}} \bullet(X ; n)) \text {. }
$$

Recall the arithmetic Chow groups defined by Gillet-Soulé [GS] as follows:

$$
\widehat{C H}^{n}(X):=\frac{\left\{(Z, g) ; \frac{\bar{\partial} \partial}{\pi i} g+\delta_{Z} \in \mathcal{A}^{n, n}\right\}}{\{(0, \partial u+\bar{\partial} v) ;(\operatorname{div} f,-\log |f|), f \in \mathbb{C}(Y), \operatorname{codim}(Y)=n-1\}} .
$$

Here $Z$ is a divisor in $X$ and $f$ is a rational function on a divisor $Y$ in $X$,

$$
g \in \mathcal{D}_{\mathbb{R}}^{n-1, n-1}(n-1), \quad(u, v) \in\left(\mathcal{D}^{n-2, n-1} \oplus \mathcal{D}^{n-1, n-2}\right)_{\mathbb{R}}(n-1) .
$$

Proposition 2.14. $\widehat{C H}^{n}(X ; 0)=\widehat{C H}^{n}(X)$.

Proof. Let us look at the very right part of the complex $\widehat{\mathcal{Z}} \bullet(X ; n)$ :

$$
\begin{aligned}
& \ldots \quad \longrightarrow \mathcal{Z}^{2 n-1}(X ; n) \longrightarrow \mathcal{Z}^{2 n}(X ; n) \\
& \downarrow \mathcal{P}^{2 n-1}(n) \quad \downarrow \mathcal{P}^{2 n}(n) \\
& \left(\mathcal{D}^{n-2, n-1} \oplus \mathcal{D}^{n-1, n-2}\right)_{\mathbb{R}}(n-1) \stackrel{(\partial, \bar{\partial})}{\longrightarrow} \mathcal{D}_{\mathbb{R}}^{n-1, n-1}(n-1) \stackrel{2 \bar{\partial} \partial}{\longrightarrow} \mathcal{D}_{\mathbb{R}}^{n, n}(n) / \mathcal{A}_{\mathbb{R}}^{n, n}(n)
\end{aligned}
$$


Consider the very end of the Gersten complex on $X$ :

$$
\prod_{Y \in X_{n-2}} \Lambda^{2} \mathbb{C}(Y)^{*} \stackrel{\partial}{\longrightarrow} \prod_{Y \in X_{n-1}} \mathbb{C}(Y)^{*} \longrightarrow \mathcal{Z}_{0}(X ; n)
$$

where $\partial$ is the tame symbol. It maps to the complex $\widehat{\mathcal{Z}} \bullet(X ; n)$, i.e., to the top row of the bicomplex above, as follows. Recall that $\mathcal{Z}_{0}(X ; n)=\mathcal{Z}^{2 n}(X ; n)$, so the very right component of our map is provided by this identification. Furthermore, a pair $(Y ; f)$ where $Y$ is an irreducible codimension $n-1$ subvariety of $X$ maps to the cycle $\{(y, f(y)) \mid y \in Y\} \subset X \times \mathbb{A}^{1}$. Similarly any element in $\Lambda^{2} \mathbb{C}(Y)^{*}$ can be represented as a linear combination of elements $\sum_{i}\left(Y ; f_{i} \wedge g_{i}\right)$ where $Y$ is an irreducible codimension $n-2$ subvariety of $X$ and $f_{i}, g_{i}$ are rational functions on $Y$ such that $\operatorname{div} f_{i}$ and $\operatorname{div} g_{i}$ share no irreducible divisors. Then we send $\left(Y ; f_{i} \wedge g_{i}\right)$ to the cycle $\left(y, f_{i}(y), g_{i}(y)\right) \subset X \times \mathbb{A}^{2}$. It is well known that in this way we get an isomorphism on the last two cohomology groups. Computing the composition of this map with the homomorphism $\mathcal{P}^{\bullet}(n)$ we end up precisely with the denominator in (38). The proposition is proved.

\section{The Chow polylogarithms}

Suppose $X=\operatorname{Spec}(\mathbb{C})$. Then $\mathcal{P}_{n}(Y):=\mathcal{P}^{1}(n)(Y)$ is a function on the space of all codimension $n$ cycles in $\mathbb{P}^{2 n-1}$ intersecting properly faces of the simplex $L$. It is called the Chow polylogarithm function. For $i>1$ all the distributions $\mathcal{P}^{i}(n)(Y)$ are zero. However, modifying the construction of the previous section we get a very interesting object, the Chow polylogarithm, even when $X$ is a point. The Chow polylogarithm function is the first component of the Chow polylogarithm. One can define the Chow polylogarithm for an arbitrary variety $X$, but we spell out the details in the most interesting case when $X$ is a point.

1. Chow polylogarithms G5]. Let $L=\left(L_{0}, \ldots, L_{p+q}\right)$ be a simplex in $\mathbb{P}^{p+q}$, $H$ a hyperplane in generic position to $L$, and $H_{i}:=H \cap L_{i}$.

Let $\mathcal{Z}_{p}^{q}(L)$ be the variety of all codimension $q$ effective algebraic cycles in $\mathbb{C P}^{p+q}$ which intersect properly, i.e., each irreducible component in the right codimension and all faces of the simplex $L$. It is a union of an infinite number of finitedimensional complex algebraic varieties.

Example. $\mathbb{C P}^{n}-L=\left(\mathbb{C}^{*}\right)^{n}$ is an irreducible component of $\mathcal{Z}_{0}^{n}(L)$ parametrizing the irreducible subvarieties, i.e., points.

Let $\widehat{L}_{i}$ be the simplex in the projective space $L_{i}$ cut by the hyperplanes $L_{j}, j \neq i$. The intersection of a cycle with a codimension 1 face $L_{i}$ of the simplex $L$ provides a map

$$
a_{i}: \mathcal{Z}_{p}^{q}(L) \longrightarrow \mathcal{Z}_{p-1}^{q}\left(\widehat{L}_{i}\right), \quad 0 \leq i \leq p+q .
$$

Let $l_{j}$ be the vertex opposite to the face $L_{j}$. Consider an open part $\mathcal{Z}_{p}^{q}(L)^{0}$ of $\mathcal{Z}_{p}^{q}(L)$ parametrizing the cycles $C$ such that projection with the center $l_{j}$ sends $C$ to a codimension $q-1$ cycle. Then projection with the center at the vertex $l_{j}$ of $L$ defines a map

$$
b_{j}: \mathcal{Z}_{p}^{q}(L)^{0} \longrightarrow \mathcal{Z}_{p}^{q-1}\left(\widehat{L}_{j}\right), \quad 0 \leq i \leq p+q
$$


Theorem-Construction 3.1. For given $q \geq 0$ there is an explicitly constructed chain of $(q-p-1)$-distributions $\omega_{p}^{q}=\omega_{p}^{q}(L ; H)$ on $\mathcal{Z}_{p}^{q}(L)$ such that

$$
\text { (i) } \quad d \omega_{0}^{q}(L, H)=\pi_{q}\left(\Omega_{L}\right),
$$$$
\text { (ii) } \quad d \omega_{p}^{q}(L ; H)=\sum_{i=0}^{p+q}(-1)^{i} a_{i}^{*} \omega_{p-1}^{q}\left(L ; H_{i}\right) \text {, }
$$$$
\text { (iii) } \quad \sum_{j=0}^{p+q+1}(-1)^{j} b_{j}^{*} \omega_{p}^{q}(L ; H)=0 .
$$

The restriction of $\omega_{p}^{q}$ to the subvariety $\widehat{\mathcal{Z}}_{p}^{q}(L)$ of smooth cycles in generic position with respect to the simplex $L$ is a real-analytic differential $(q-p-1)$-form.

For a given positive integer $q$ the collection $\left\{\omega_{p}^{q}\right\}$ is called the $q$-th Chow polylogarithm.

The varieties $\mathcal{Z}_{p}^{q}(L)$ for $p \geq 0$ form a truncated simplicial variety $\mathcal{Z}_{\bullet}^{q}(L)$. The conditions (i) and (ii) just mean that the sequence of forms $\omega_{p}^{q}$ is a $2 q$-cocycle in the complex computing the Deligne cohomology $H^{2 q}\left(\mathcal{Z}_{\bullet}^{q}(L), \mathbb{R}_{\mathcal{D}}(q)\right)$.

Proof. We define $\omega_{p}^{q}$ as the Radon transform of the distribution $r_{p+q-1}(L ; H)$ in $\mathbb{C P}^{p+q}$ over the family of cycles $Y_{\xi}$ parametrized by $\mathcal{Z}_{p}^{q}(L)$. This means the following. Consider the incidence variety:

$$
\Gamma_{p}:=\left\{(x, \xi) \in \mathbb{C P}^{p+q} \times \mathcal{Z}_{p}^{q}(L)(\mathbb{C}) \text { such that } x \in Y_{\xi}\right\}
$$

where $Y_{\xi}$ is the cycle in $\mathbb{C P}^{p+q}$ corresponding to $\xi \in \mathcal{Z}_{p}^{q}(L)$. We get a double bundle

$$
\Gamma_{p} \subset \mathbb{C P}^{p+q} \times \mathcal{Z}_{p}^{q}(L)(\mathbb{C})
$$

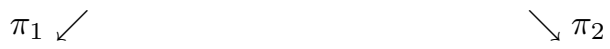

$$
\mathbb{C P}^{p+q} \quad \mathcal{Z}_{p}^{q}(L)(\mathbb{C})
$$

Then

$$
\omega_{p}^{q}:=\pi_{2 *} \operatorname{Res}_{\Gamma_{p}} \pi_{1}^{*}(2 \pi i)^{-q} r_{p+q}(L ; H) .
$$

Observe that $r_{p+q}(L ; H)$ is a distribution on $\mathbb{C P}^{p+q}$, and hence $\pi_{1}^{*} r_{p+q}(L ; H)$ is a distribution on $\mathbb{C P}^{p+q} \times \mathcal{Z}_{p}^{q}(L)(\mathbb{C})$. The fact that this distribution can be restricted to $\Gamma_{p}$ is a version of Theorem 2.4 and is proved in the same way. The push-forward $\pi_{2 *}$ of this distribution is well-defined since $\pi_{2}$ is a proper map.

The property (i) is true by the very definition.

Lemma 3.2. $\sum_{j=0}^{n+1}(-1)^{j} b_{j}^{*} \omega_{0}^{n}=0$.

Proof. Let $s\left(z_{0}, \ldots, z_{n}\right):=z_{1} / z_{0} \wedge \ldots \wedge z_{n} / z_{0}$. The lemma follows from the identity

$$
\sum_{j=0}^{n+1}(-1)^{j} s\left(z_{0}, \ldots, \widehat{z_{j}}, \ldots, z_{n+1}\right)=0 .
$$

So we have (iii). To check (ii) observe that the push-forward $\pi_{2 *}$ of distributions commutes with the de Rham differential. The theorem is proved. 
2. Properties of the Chow polylogarithm function. The function $\mathcal{P}_{q}:=$ $\omega_{q-1}^{q}$ on $\mathcal{Z}_{q-1}^{q}(L)$ is called the Chow q-logarithm function. It satisfies two functional equations:

$$
\sum_{i=0}^{2 q}(-1)^{i} a_{i}^{*} \mathcal{P}_{q}=0, \quad \sum_{j=0}^{2 q}(-1)^{j} b_{j}^{*} \mathcal{P}_{q}=0 .
$$

Theorem 3.3. The Chow polylogarithm function is invariant under the natural action of the torus $\left(\mathbb{C}^{*}\right)^{p+q}$ on $\mathcal{Z}_{p}^{q}(\mathbb{C})$. In particular, it does not depend on the choice of the hyperplane $H$.

Remark. The statements of Theorem 3.3 are no longer true for the forms $\omega_{p}^{q}$ for $p<q-1$.

Here is a reformulation of Theorem 3.3

Theorem 3.4. Suppose that $\operatorname{dim} X=n$ and $f_{1}, \ldots, f_{2 n+1}$ are rational functions on $X$. Then the integral

$$
(2 \pi i)^{1-n} \int_{X(\mathbb{C})} r_{2 n}\left(f_{1}, \ldots, f_{2 n+1}\right)
$$

does not change if we multiply one of the functions $f_{i}$ by a nonzero constant.

Proof. Multiplying, say, $f_{1}$ by $\lambda$ we see that the difference between the two integrals is

(42) $\log |\lambda| \sum_{k} a_{k} \int_{X(\mathbb{C})} \operatorname{Alt}_{2 n} d \log \left|f_{2}\right| \wedge \ldots \wedge d \log \left|f_{2 k-1}\right| \wedge d \arg f_{2 k} \wedge \ldots \wedge d \arg f_{2 n+1}$

where the $a_{k}$ are some rational constants (easily computable from (15)). We will prove that for each $k$ the corresponding integral in this sum is already zero. Using the identity

$$
\left(d \log \left|f_{2}\right|+i d \arg f_{2}\right) \wedge \ldots \wedge\left(d \log \left|f_{2 n+1}\right|+i d \arg f_{2 n+1}\right)=0
$$

we can rewrite the integral

$$
\int_{X(\mathbb{C})} d \arg f_{2} \wedge \ldots \wedge d \arg f_{2 n+1}
$$

as a sum of similar integrals containing $d \log \left|f_{i}\right|$. Our statement follows from Proposition 3.5.

Proposition 3.5. Suppose that $\operatorname{dim} X=n$. Then

$$
\begin{aligned}
& d \operatorname{Alt}_{2 n}\left(\log \left|f_{2}\right| d \log \left|f_{3}\right| \wedge \ldots \wedge d \log \left|f_{2 k-1}\right| \wedge d \arg f_{2 k} \wedge \ldots \wedge d \arg f_{2 n+1}\right) \\
& =\operatorname{Alt}_{2 n}\left(d \log \left|f_{2}\right| \wedge \ldots \wedge d \log \left|f_{2 k-1}\right| \wedge d \arg f_{2 k} \wedge \ldots \wedge d \arg f_{2 n+1}\right)
\end{aligned}
$$

in the sense of distributions.

Proof. Since $d d i \arg f=2 \pi i \delta(f)$, the left-hand side is equal to the right-hand side plus the following terms concentrated on the divisors $f_{2 k+j}=0$ :

$$
\begin{aligned}
4 \pi \cdot 2(n-k+1) \operatorname{Alt}_{2 n}\left(\delta\left(f_{2 n+1}\right) \log \left|f_{2}\right| d \log \left|f_{3}\right|\right. & \wedge \ldots \wedge d \log \left|f_{2 k-1}\right| \\
& \left.\wedge d \arg f_{2 k} \wedge \ldots \wedge d \arg f_{2 n}\right) .
\end{aligned}
$$

However, all these additional terms vanish thanks to the following proposition. 
Proposition 3.6. Suppose that $\operatorname{dim} X=n$. Then for each $0 \leq j \leq n-1$ one has

$$
\begin{gathered}
\operatorname{Alt}_{2 n}\left(d \log \left|f_{1}\right| \wedge \ldots \wedge d \log \left|f_{2 j+1}\right| \wedge d \arg f_{2 j+2} \wedge \ldots \wedge d \arg f_{2 n}\right)=0, \\
\operatorname{Alt}_{2 n}\left(d \log \left|f_{1}\right| \wedge \ldots \wedge d \log \left|f_{2 j}\right| \wedge d \arg f_{2 j+1} \wedge \ldots \wedge d \arg f_{2 n}\right) \\
=\frac{(2 n) !\left(\begin{array}{c}
n \\
j
\end{array}\right)}{\left(\begin{array}{c}
2 n \\
2 j
\end{array}\right)} \cdot d \log \left|f_{1}\right| \wedge \ldots \wedge d \log \left|f_{2 n}\right| .
\end{gathered}
$$

Proof. The idea is this. One has $n$ equations:

$$
\begin{gathered}
d \log f_{1} \wedge \ldots \wedge d \log f_{2 n}=0, \\
d \log \left|f_{1}\right| \wedge d \log f_{2} \wedge \ldots \wedge d \log f_{2 n}=0,
\end{gathered}
$$

$$
d \log \left|f_{1}\right| \wedge \ldots \wedge d \log \left|f_{n-1}\right| \wedge d \log f_{n} \wedge \ldots \wedge d \log f_{2 n}=0 .
$$

Taking the imaginary part of each of them and alternating $f_{1}, \ldots, f_{2 n}$ we get $n$ linear equations. Solving them we get the proposition. See the details in the Appendix in $\mathrm{GZ}$.

\section{The Grassmannian polylogarithms}

1. Configurations of vectors and Grassmannians: a dictionary. Let $G$ be a group. Let $X$ be a $G$-set. We define configurations of $m$ points of $X$ as $G$-orbits in $X^{m}$.

Example 1. If $X:=V$ is a vector space and $G:=G L(V)$ we get configurations of vectors in $V$. A configuration of vectors $\left(l_{1}, \ldots, l_{m}\right)$ is in generic position if each $k \leq \operatorname{dim} V$ of the vectors are linearly independent.

Example 2. If $X:=\mathbb{P}(V)$ is a projective space and $G:=P G L(V)$ we get configurations $\left(x_{1}, \ldots, x_{m}\right)$ of $m$ points in $\mathbb{P}(V)$. A configuration of points is in generic position if each $k \leq \operatorname{dim} V$ of them generate a plane of dimension $k-1$.

Let $T_{p+q}$ be the quotient of the torus $\mathbb{G}_{m}^{p+q+1}$ by the diagonal subgroup $\mathbb{G}_{m}=$ $(t, \ldots, t)$. In what follows $V_{n}$ denotes a vector space of dimension $n$.

Lemma-Construction 4.1. (i) There are canonical isomorphisms between the following sets of geometric objects:

(a) configurations of $p+q+1$ vectors in generic position in $V_{q}$;

(b) isomorphism classes of triples $\left\{\right.$ a projective space $\mathbb{P}^{p+q}$ together with a simplex L, an "infinite" hyperplane $H$ in generic position to $L$, and a p-dimensional plane in generic position to $L$ (but not necessarily to $H$ ) \};

(c) isomorphism classes of triples $\left\{\right.$ a vector space $V_{p+q+1}$, a basis $\left(e_{0}, \ldots, e_{p+q}\right)$ of $V_{p+q+1}$, and a $(p+1)$-dimensional subspace of $V_{p+q+1}$ in generic position with respect to the coordinate hyperplanes\}.

(ii) The torus $T_{p+q}$ acts naturally, and without fixed points, on each of the objects (a), (b), (c), and the isomorphisms above are compatible with this action.

Proof. (i) (a) $\rightarrow$ (c). For the $(p+1)$-dimensional subspace, take the kernel of the linear map from $V_{p+q+1}$ to $V_{q}$ sending $e_{i}$ to $l_{i}$.

(c) $\rightarrow$ (a). Take the quotient of $V_{p+q+1} / h$ along the given subspace $h$ and consider the images of the vectors $\left(e_{0}, \ldots, e_{p+q}\right)$ there.

$(\mathrm{c}) \rightarrow(\mathrm{b})$. Let $\mathbb{P}^{p+q}:=\mathbb{P}\left(V_{p+q+1}\right)$. Let $\mathbb{A}^{p+q}$ be the affine hyperplane in $V_{p+q+1}$ passing through the ends of the basis vectors $e_{i}$. Then $\mathbb{A}^{p+q} \subset \mathbb{P}^{p+q}$. The coordinate 
hyperplanes in $V_{p+q+1}$ provide a simplex $L_{p+q} \subset \mathbb{P}^{p+q}$. The projectivization of a generic $(p+1)$-dimensional subspace $h$ in $V_{p+q+1}$ gives a $p$-plane $\bar{h}$ in generic position with respect to this simplex. (Notice that we do not impose any condition on the mutual location of $H$ and $\bar{h}$. For instance $\bar{h}$ may be inside of $H$.)

$(\mathrm{b}) \rightarrow(\mathrm{c})$. The triple $\left(\mathbb{P}^{p+q}, H, L\right)$ provides a unique up to an isomorphism data $\left(V_{p+q+1},\left(e_{0}, \ldots, e_{p+q}\right)\right)$. Namely, the partial data $\left(\mathbb{P}^{p+q}, H\right)$ provides us with $\left(V_{p+q+1}, \widetilde{H}\right)$ where $\widetilde{H}$ is the subspace of $V_{p+q+1}$ projecting to $H$. Now the vertices $l_{i}$ of the simplex $L$ provide coordinate lines $\widetilde{l}_{i}$ in $V_{p+q+1}$. Intersecting these coordinate lines with a parallel shift of the subspace $\widetilde{H}$ we get a point on each of the coordinate lines. By definition the endpoints of the basis vectors $e_{i}$ are these points. Taking the subspace $\widetilde{h}$ in $V_{p+q+1}$ projecting to a given $p$-plane $h$ in $\mathbb{P}^{p+q}$ we get the desired correspondence.

(ii) The torus $T_{p+q}$ acts on the configurations of vectors in (a) as

$$
\left(t_{1}, \ldots, t_{p+q+1}\right):\left(l_{1}, \ldots, l_{p+q+1}\right) \longmapsto\left(t_{1} l_{1}, \ldots, t_{p+q+1} l_{p+q+1}\right) .
$$

The torus $T_{p+q}$ is identified with $\mathbb{P}^{p+q}-L$ in (b), and so acts naturally on the data in (b). The action on the data in (c) is similar. The lemma is proved.

If we use the description (c) for the Grassmannians, then $b_{j}$ is obtained by factorization along the coordinate axis $\left\langle e_{j}\right\rangle$.

2. The Grassmannian and bi-Grassmannian polylogarithms. Let us fix a positive integer $q$. The operations $a_{i}$ and $b_{j}$ from Subsection 1 in Section 3 transform planes to planes. So we get the following diagram of varieties called the bi-Grassmannian $\widehat{G}(q)$ :

$$
\begin{aligned}
& \downarrow \ldots \downarrow \quad \downarrow \ldots \downarrow \\
& \widehat{G}(q):=\quad \stackrel{\rightarrow}{\rightarrow} \widehat{G}_{1}^{q+1} \stackrel{\dddot{\longrightarrow}}{\rightarrow} \widehat{G}_{0}^{q+1} \\
& \downarrow \ldots \downarrow \quad \downarrow \ldots \downarrow \quad \downarrow \ldots \downarrow \\
& \ldots \stackrel{\rightarrow}{\rightarrow} \quad \widehat{G}_{2}^{q} \quad \stackrel{\rightarrow}{\rightarrow} \quad \widehat{G}_{1}^{q} \quad \stackrel{\rightarrow}{\rightarrow} \quad \widehat{G}_{0}^{q}
\end{aligned}
$$

Here the horizontal arrows are the maps $a_{i}$ and the vertical ones are $b_{j}$.

Remark. The bi-Grassmannian $\widehat{G}(n)$ is not a (semi)bisimplicial scheme. (It is a truncated semihypersimplicial scheme. See Section 2.6 in [G4].)

Configurations of hyperplanes and torus quotients of Grassmannians. Let $\widehat{G}_{p}^{q}$ be the Grassmannian of $p$-planes in $\mathbb{P}^{p+q}$ in generic position with respect to a given simplex $L$.

Taking the $T_{p+q}$-orbits of the objects (a) and (b) in the lemma we arrive at

Corollary 4.2. There is a bijective correspondence

$$
\widehat{G}_{p}^{q} / T_{p+q}<-->\left\{\text { Configurations of } p+q+1 \text { generic hyperplanes in } \mathbb{P}^{p}\right\}
$$

sending a p-plane $h$ to the configuration $\left(h \cap L_{0}, \ldots, h \cap L_{p+q}\right)$ in $h$.

Let $\psi_{p}^{q}(q)$ be the restriction of the differential form $\omega_{p}^{q}$ to $\widehat{G}_{p}^{q}$. The properties (i), (ii) from Theorem 3.1 are exactly the defining conditions for the single-valued 
Grassmannian polylogarithm whose existence was conjectured in [HM], [BMS]; see also [GGL].

Let us extend these forms by zero to the other rows of the bi-Grassmannian $\widehat{G}(q)$, i.e., set $\psi_{p}^{q+i}(q)=0$ if $i>0$. Then the property (iii) from Theorem 3.1 guarantees that the forms $\psi_{p}^{q+i}(q)$ form a $2 q$-cocycle in the bicomplex computing the Deligne cohomology $H^{2 q}\left(\widehat{G}(q) \bullet, \mathbb{R}(q)_{\mathcal{D}}\right)$. This is called the bi-Grassmannian q-logarithm ([G5]).

A sequence of multivalued analytic forms on Grassmannians satisfying conditions similar to (i), (ii) was defined in HM1], [HM2]. Another construction of the multivalued analytic Grassmannian polylogarithms was suggested in [G5] in the more general setting of the multivalued Chow polylogarithm.

3. The Grassmannian $n$-logarithm function. By Theorem 3.4 the Chow polylogarithm function is invariant under the action of the torus $\left(\mathbb{C}^{*}\right)^{2 n-1}$. So restricting it to the open Grassmannian $\widehat{G}_{n-1}^{n} \subset \widehat{\mathcal{Z}}_{n-1}^{n}$ and using the bijection

$$
\begin{gathered}
\left\{(n-1) \text {-planes in } \mathbb{P}^{2 n-1}\right. \text { in generic position } \\
\text { with respect to a simplex } L\} /\left(\mathbb{G}_{m}^{*}\right)^{2 n-1} \\
<-->\quad\left\{\text { Configurations of } 2 n \text { generic hyperplanes in } \mathbb{P}^{n-1}\right\}
\end{gathered}
$$

we get a function on the configurations of $2 n$ hyperplanes in $\mathbb{C P}^{n-1}$, called the Grassmannian polylogarithm function $\mathcal{L}_{n}^{G}$.

The Grassmannian polylogarithm function has the following simple description on the language of configurations of hyperplanes. It is interesting that in this description we can work with any configuration of $2 n$ hyperplanes, assuming nothing about their mutual location.

Let $h_{1}, \ldots, h_{2 n}$ be $2 n$ arbitrary hyperplanes in $\mathbb{C P}^{n-1}$. Choose an additional hyperplane $h_{0}$. Let $f_{i}$ be a rational function on $\mathbb{C P}^{n-1}$ with divisor $\left(h_{i}\right)-\left(h_{0}\right)$. It is defined up to a scalar factor. Set

$$
\mathcal{L}_{n}^{G}\left(h_{1}, \ldots, h_{2 n}\right):=(2 \pi i)^{1-n} \int_{\mathbb{C P}^{n-1}} r_{2 n-2}\left(\sum_{j=1}^{2 n}(-1)^{j} f_{1} \wedge \ldots \wedge \widehat{f}_{j} \wedge \ldots \wedge f_{2 n}\right) .
$$

It is skewsymmetric by definition. Notice that

$$
\sum_{j=1}^{2 n}(-1)^{j} f_{1} \wedge \ldots \wedge \widehat{f}_{j} \wedge \ldots \wedge f_{2 n}=\frac{f_{1}}{f_{2 n}} \wedge \frac{f_{2}}{f_{2 n}} \wedge \ldots \wedge \frac{f_{2 n-1}}{f_{2 n}} .
$$

So we can define $\mathcal{L}_{n}^{G}\left(h_{1}, \ldots, h_{2 n}\right)$ as follows: choose rational functions $g_{1}, \ldots, g_{2 n-1}$ such that $\operatorname{div} g_{i}=\left(h_{i}\right)-\left(h_{2 n}\right)$ and put

$$
\mathcal{L}_{n}^{G}\left(h_{1}, \ldots, h_{2 n}\right)=(2 \pi i)^{1-n} \int_{\mathbb{C P}^{n-1}} r_{2 n-2}\left(g_{1}, \ldots, g_{2 n-1}\right) .
$$

Remark. The function $\mathcal{L}_{n}^{G}$ is defined on the set of all configurations of $2 n$ hyperplanes in $\mathbb{C P}^{n-1}$. However, it is not even continuous on this set. It is real analytic on the submanifold of generic configurations. Since we put no restrictions on the hyperplanes $h_{i}$ the following theorem is stronger than Theorem 3.1 in the case of linear subvarieties.

Theorem 4.3. The function $\mathcal{L}_{n}^{G}$ has the following properties:

(a) It does not depend on the choice of hyperplane $h_{0}$. 
(b) For any $2 n+1$ hyperplanes $\left(h_{1}, \ldots, h_{2 n+1}\right)$ in $\mathbb{C P}^{n}$ one has

$$
\sum_{j=1}^{2 n+1}(-1)^{j} \mathcal{L}_{n}^{G}\left(h_{j} \cap h_{1}, \ldots,{\widehat{h_{j} \cap h_{j}}}_{j}, \ldots, h_{j} \cap h_{2 n+1}\right)=0 .
$$

(c) For any $2 n+1$ hyperplanes $\left(h_{1}, \ldots, h_{2 n+1}\right)$ in $\mathbb{C P}^{n-1}$ one has

$$
\sum_{j=1}^{2 n+1}(-1)^{j} \mathcal{L}_{n}^{G}\left(h_{1}, \ldots, \widehat{h}_{j}, \ldots, h_{2 n+1}\right)=0 .
$$

Proof. (a) Choose another hyperplane $h_{0}^{\prime}$. Take a rational function $f_{0}$ with divisor $\left(h_{0}^{\prime}\right)-\left(h_{0}\right)$. Set $f_{i}^{\prime}=\frac{f_{i}}{f_{0}}$. Then

$$
\sum_{j=1}^{2 n+1}(-1)^{j} f_{1} \wedge \ldots \wedge \widehat{f}_{j} \wedge \ldots \wedge f_{2 n+1}-\sum_{j=1}^{2 n+1}(-1)^{j} f_{1}^{\prime} \wedge \ldots \wedge \widehat{f}_{j}^{\prime} \wedge \ldots \wedge f_{2 n+1}^{\prime}=0 .
$$

Indeed, substituting $f_{i}^{\prime}=\frac{f_{i}}{f_{0}}$ in this formula we find that the only possible nontrivial term $f_{0} \wedge f_{1} \wedge \ldots \wedge \widehat{f}_{i} \wedge \ldots \wedge \widehat{f}_{j} \wedge \ldots \wedge f_{2 n}$ vanishes because it is symmetric in $i, j$.

(b) Let $g_{1}, \ldots, g_{2 n+1}$ be rational functions on $\mathbb{C P}^{n}$ with $\operatorname{div} g_{i}=\left(h_{i}\right)-\left(h_{0}\right)$. Then

$$
\begin{gathered}
d r_{2 n-1}\left(\sum_{j=1}^{2 n+1}(-1)^{j} g_{1} \wedge \ldots \wedge \widehat{g}_{j} \wedge \ldots \wedge g_{2 n+1}\right) \\
=\sum_{j \neq i}(-1)^{j+i-1} 2 \pi i \delta\left(f_{i}\right) \wedge r_{2 n-2}\left(g_{1} \wedge \ldots \wedge \widehat{g}_{i} \wedge \ldots \wedge \widehat{g}_{j} \wedge \ldots \wedge g_{2 n+1}\right) .
\end{gathered}
$$

(Notice that $d \log g_{1} \wedge \ldots \wedge \widehat{d \log g_{j}} \wedge \ldots \wedge d \log g_{2 n+1}=0$ on $\mathbb{C P}^{n}$.) Integrating (49) over $\mathbb{C P}^{n}$ we see that the left-hand side equals zero, while the right-hand side equals the sum of the expressions staying on the left of (47). So we get (b).

(c) is obvious: we apply $r_{2 n-2}$ to the zero element. The theorem is proved.

4. $\mathbb{P}^{1} \backslash\{0, \infty\}$ as a special stratum in the configuration space of $2 n$ points in $\mathbb{P}^{n-1}$. A special configuration is a configuration of $2 n$ points

$$
\left(l_{0}, \ldots, l_{n-1}, m_{0}, \ldots, m_{n-1}\right)
$$

in $\mathbb{P}^{n-1}$ such that $l_{0}, \ldots, l_{n-1}$ are vertices of a simplex in $\mathbb{P}^{n-1}$ and $m_{i}$ is a point on the edge $l_{i} l_{i+1}$ of the simplex different from $l_{i}$ and $l_{i+1}$, as in Figure 17

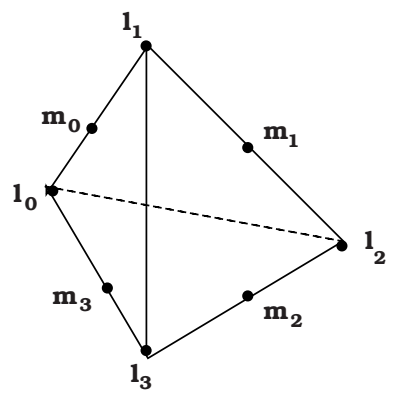

FIGURE 7. A special configuration of 8 points in $\mathbb{P}^{3}$ 
Proposition 4.4. The set of special configurations of $2 n$ points in $\mathbb{P}^{n-1}$ is canonically identified with $\mathbb{P}^{1} \backslash\{0, \infty\}$. (See Figure 8.)

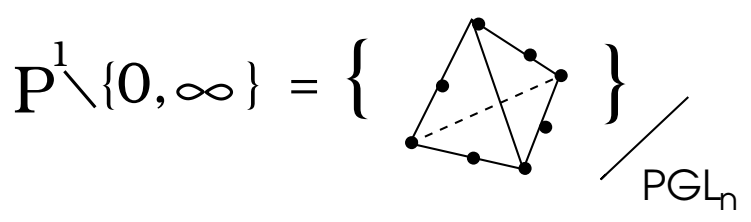

FiguRE 8. $\mathbb{P}^{1} \backslash\{0, \infty\}$ is a stratum in the configuration space of $2 n$ points in $\mathbb{P}^{n-1}$.

Proof. We define the generalized cross-ratio

$$
r\left(l_{0}, \ldots, l_{n-1}, m_{0}, \ldots, m_{n-1}\right) \in F^{*}
$$

where $F$ is the common field of definition of the points $l_{i}, m_{j}$, as follows (see Figure 9). Consider the one-dimensional subspaces $L_{i}, M_{j}$ in the $n$-dimensional vector space $V$ projecting to the points $l_{i}, m_{j}$ in $\mathbb{P}^{n-1}$ respectively. The subspaces $L_{i}, M_{i}, L_{i+1}$ generate a two-dimensional subspace. Its quotient along $M_{i}$ can be identified with $L_{i}$ as well as with $L_{i+1}$. So we get a canonical linear map $\bar{M}_{i}: L_{i} \longrightarrow L_{i+1}$. The composition of these maps (the "linear monodromy")

$$
\bar{M}_{0} \circ \ldots \circ \bar{M}_{n-1}: L_{0} \longrightarrow L_{0}
$$

is multiplication by an element of $F^{*}$ called the generalized cross-ratio of the special configuration (50).


Figure 9. The generalized cross-ratio of a special configuration

It is clearly invariant under the cyclic permutation

$$
l_{0} \rightarrow l_{0} \rightarrow \ldots \rightarrow l_{n-1} \rightarrow l_{0} ; \quad m_{0} \rightarrow m_{1} \rightarrow \ldots \rightarrow m_{n-1} \rightarrow m_{0} .
$$

Notice that $r\left(l_{0}, \ldots, l_{n-1}, m_{0}, \ldots, m_{n-1}\right)=1$ if and only if the points $m_{0}, \ldots, m_{n-1}$ belong to a hyperplane. 
Let $\widehat{m}_{i}$ be the point of intersection of the line $l_{i} l_{i+1}$ with the hyperplane passing through all the points $m_{j}$ except $m_{i}$. Let $r\left(x_{1}, \ldots, x_{4}\right)$ be the cross-ratio of the four points $x_{i}$ on $\mathbb{P}^{1}$. Then

$$
r\left(l_{0}, \ldots, l_{n-1}, m_{0}, \ldots, m_{n-1}\right)=r\left(l_{i}, l_{i+1}, m_{i}, \widehat{m}_{i+1}\right) .
$$

The proof of the proposition follows from these constructions.

The special configurations and classical polylogarithms. Consider the configuration of $2 n$ hyperplanes in $\mathbb{P}^{n-1}$ given by the following equations in homogeneous coordinates $z_{0}: \ldots: z_{n-1}$ :

$$
\begin{gathered}
z_{0}=0, \quad \ldots, \quad z_{n-1}=0, \quad z_{0}=z_{1}, \quad z_{1}+z_{2}=z_{0}, \\
z_{2}-z_{3}=0, \quad \ldots, \quad z_{n-2}-z_{n-1}=0, \quad z_{n-1}=a z_{0} .
\end{gathered}
$$

It admits the following interpretation. Recall that the classical polylogarithm function $L i_{n-1}(z)$ can be defined by an iterated integral:

$$
L i_{n-1}(a)=\int_{0}^{a} \frac{d t}{1-t} \circ \frac{d t}{t} \circ \ldots \circ \frac{d t}{t}=\int_{\Delta_{a}} \frac{d z_{1}}{z_{1}} \wedge \ldots \wedge \frac{d z_{n-1}}{z_{n-1}} .
$$

If $a \in(0,1]$, then the simplex $\Delta_{a}$ is defined by the equations

$$
\Delta_{a}:=\quad\left\{\left(z_{1}, \ldots, z_{n-1}\right) \in \mathbb{R}^{n-1} \mid \quad 0 \leq 1-z_{1} \leq z_{2} \leq z_{3} \leq \ldots \leq z_{n-1} \leq a\right\} .
$$

The faces of the simplex $\Delta_{a}$ can be defined for arbitrary $a$. Then the codimension one faces $\left\{z_{i}=0\right\}$ of the coordinate simplex and the codimension one faces of the simplex $\Delta_{a}$ form the configuration (51).

We can reorder hyperplanes of this configuration as follows:

$$
\begin{gathered}
z_{0}=0, \quad z_{1}=0, \quad z_{1}=z_{0}, \quad z_{1}+z_{2}=z_{0}, \quad z_{2}=0 \\
z_{2}=z_{3}, \quad z_{3}=0, \quad \ldots \quad, \quad z_{n-2}=0, \quad z_{n-2}=z_{n-1}, \quad z_{n-1}=a z_{0} .
\end{gathered}
$$

Applying the projective duality to this configuration of hyperplanes we get the special configuration of $2 n$ points in $\mathbb{P}^{n-1}$ with the generalized cross-ratio $a$.

The correspondence between the configuration (51) and the special configuration of points is illustrated in the case $n=3$ in Figure 10,
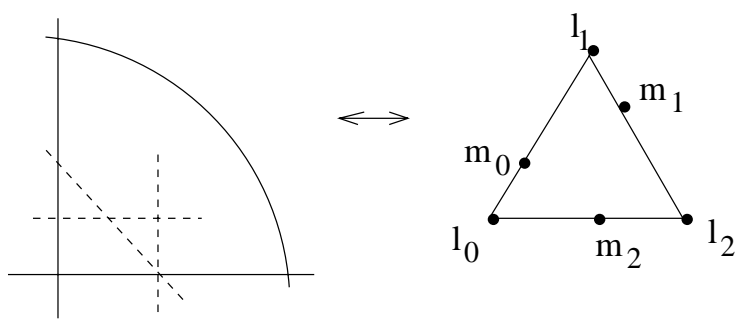

FiguRE 10. Classical polylogarithm configurations and special configurations

Remark. It is amusing that the special configuration of $2 n$ points in $\mathbb{P}^{n-1}$, which is related to the classical $n$-logarithm by Theorem 4.5 below, is constructed using the geometry of the mixed motive corresponding to $L i_{n-1}(a)$. 


\section{Restriction of the Grassmannian $n$-logarithm to the special stratum.} The function $L i_{n}(z)$ has a remarkable single-valued version ([Z1], [BD] $)$ :

$$
\mathcal{L}_{n}(z):=\begin{aligned}
& \operatorname{Re}(n: \text { odd }) \\
& \operatorname{Im} \quad(n: \text { even })
\end{aligned}\left(\sum_{k=0}^{n-1} \beta_{k} \log ^{k}|z| \cdot L i_{n-k}(z)\right), \quad n \geq 2 .
$$

It is continuous on $\mathbb{C P}^{1}$. Here $\frac{2 x}{e^{2 x}-1}=\sum_{k=0}^{\infty} \beta_{k} x^{k}$, so $\beta_{k}=\frac{2^{k} B_{k}}{k !}$ where the $B_{k}$ are the Bernoulli numbers. For example, $\mathcal{L}_{2}(z)$ is the Bloch-Wigner function.

Let us consider the following modification of the function $\mathcal{L}_{n}(z)$ proposed by A. M. Levin in Le:

$$
\begin{gathered}
\widetilde{\mathcal{L}}_{n}(x):= \\
\frac{(2 n-3)}{(2 n-2)} \sum_{k \text { even; } 0 \leq k \leq n-2} \frac{2^{k}(n-2) !(2 n-k-3) !}{(2 n-3) !(k+1) !(n-k-2) !} \mathcal{L}_{n-k}(x) \log ^{k}|x| .
\end{gathered}
$$

For example, $\widetilde{\mathcal{L}}_{n}(x)=\mathcal{L}_{n}(x)$ for $n \leq 3$, but already $\widetilde{\mathcal{L}}_{4}(x)$ is different from $\mathcal{L}_{4}(x)$. A direct integration carried out in Proposition 4.4.1 of [Le] shows that

$$
\begin{gathered}
-(2 \pi i)^{n-1}(-1)^{(n-1)(n-2) / 2} \widetilde{\mathcal{L}}_{n}(x) \\
=\int_{\mathbb{C P}^{n-1}} \log \left|1-z_{1}\right| \prod_{i=1}^{n-1} d \log \left|z_{i}\right| \wedge \prod_{i=1}^{n-2} d \log \left|z_{i}-z_{i+1}\right| \wedge d \log \left|z_{n-1}-a\right| .
\end{gathered}
$$

This combined with Proposition 5.3 below implies

Theorem 4.5. The value of the function $\mathcal{L}_{n}^{G}$ on the special configuration (50) is equal to

$$
-(-1)^{n(n-1) / 2} 4^{n-1}\left(\begin{array}{c}
2 n-2 \\
n-1
\end{array}\right)^{-1} \widetilde{\mathcal{L}}_{n}(a)
$$

where $a=r\left(l_{0}, \ldots, l_{n-1}, m_{0}, \ldots, m_{n-1}\right)$.

Another proof in the case $n=2$ is given in Proposition 6.8.

Conjecture 4.6. The Chow n-logarithm function can be expressed by the Grassmannian n-logarithm function.

Remark. Suppose that an element $\sum_{k}\left\{f_{1}^{(k)}, \ldots, f_{2 n+1}^{(k)}\right\} \in K_{2 n+1}^{M}(\mathbb{C}(X))$ has zero residues at all the divisors on an $n$-dimensional variety $X$ over $\mathbb{C}$. Then it defines an element

$$
\alpha \in g r_{2 n+1}^{\gamma} K_{2 n+1}(X)=\operatorname{Ext}_{\mathcal{M}}^{2 n+1}\left(\mathbb{Q}(0)_{X}, \mathbb{Q}(2 n+1)_{X}\right) .
$$

Its direct image to the point is an element

$$
\pi_{*}(\alpha) \in g r_{n}^{\gamma} K_{2 n+1}(\operatorname{Spec} \mathbb{C})=\operatorname{Ext}_{\mathcal{M}}^{1}(\mathbb{Q}(0), \mathbb{Q}(n)) .
$$

Applying the regulators we see that the integral $\sum_{k} \int_{X(\mathbb{C})} r_{2 n}\left(f_{1}^{(k)}, \ldots, f_{2 n+1}^{(k)}\right)$ coincides, up to a factor, with the value of the Borel regulator map on $\pi_{*}(\alpha)$ and so by results of the next section is expressible by the Grassmannian $n$-logarithms. Conjecture 4.6 tells us that this should be true for any element in $K_{2 n+1}^{M}(\mathbb{C}(X))$. 


\section{Grassmannian polylogarithms, Symmetric spaCes AND BOREL REGULATORS}

1. The function $\psi_{n}$. Let $V_{n}$ be a complex vector space of dimension $n$. Let

$\mathbb{H}_{n}:=\left\{\right.$ positive definite Hermitian forms in $\left.V_{n}\right\} / \mathbb{R}_{+}^{*}=S L_{n}(\mathbb{C}) / S U(n)$

$=\left\{\right.$ positive definite Hermitian forms in $V_{n}$ with determinant $\left.=1\right\}$.

It is a symmetric space of rank $n-1$. For example, $\mathbb{H}_{2}=\mathcal{H}_{3}$ is the hyperbolic 3-space. Replacing positive definite by nonnegative definite Hermitian forms we get a compactification $\overline{\mathbb{H}}_{n}$ of the symmetric space $\mathbb{H}_{n}$.

Let $G_{x}$ be the subgroup of $S L_{N}(\mathbb{C})$ stabilizing the point $x \in \mathbb{H}_{n}$. A point $x$ defines a one-dimensional vector space $M_{x}$ :

$$
x \in \mathbb{H}_{n} \longmapsto M_{x}:=\left\{\text { measures on } \mathbb{C P}^{n-1} \text { invariant under } G_{x}\right\} .
$$

Namely, a point $x$ corresponds to a Hermitian metric in $V_{n}$. This metric provides the Fubini-Studi metric on $\mathbb{C P}^{n-1}=P\left(V_{n}\right)$. Moreover there is the Fubini-Studi Kähler form on $\mathbb{C P}^{n-1}=P\left(V_{n}\right)$; its imaginary part is a symplectic form. Raising it to the $(n-1)$-th power we get the Fubini-Studi volume form. The elements of $M_{x}$ are the multiples of the Fubini-Studi volume form.

So $\mathbb{H}_{n}$ embeds into the projectivization of the space of all measures in $\mathbb{C P}^{n-1}$. Taking its closure we get a compactification of $\mathbb{H}_{n}$.

Let us choose for any point $x \in \mathbb{H}_{n}$ an invariant measure $\mu_{x} \in M_{x}$. Then, for any $y \in \mathbb{H}_{n}$, the ratio $\mu_{x} / \mu_{y}$ is a real function on $\mathbb{C P}^{n-1}$.

Let $x_{0}, \ldots, x_{2 n-1}$ be points of the symmetric space $S L_{n}(\mathbb{C}) / S U(n)$. Consider the following function:

$$
\psi_{n}\left(x_{0}, \ldots, x_{2 n-1}\right):=\int_{\mathbb{C P}^{n-1}} \log \left|\frac{\mu_{x_{1}}}{\mu_{x_{0}}}\right| d \log \left|\frac{\mu_{x_{2}}}{\mu_{x_{0}}}\right| \wedge \ldots \wedge d \log \left|\frac{\mu_{x_{2 n-1}}}{\mu_{x_{0}}}\right| .
$$

2. General properties of the function $\psi_{n}$. Let us study the properties of integral (52) in a more general situation. Let $X$ be an $m$-dimensional manifold. For any $m+2$ measures $\mu_{0}, \ldots, \mu_{m+1}$ on $X$ such that $\frac{\mu_{i}}{\mu_{j}}$ are smooth functions we can construct a differential $m$-form on $X$ :

$$
\bar{r}_{m}\left(\mu_{0}: \ldots: \mu_{m+1}\right):=\log \left|\frac{\mu_{1}}{\mu_{0}}\right| d \log \left|\frac{\mu_{2}}{\mu_{0}}\right| \wedge \ldots \wedge d \log \left|\frac{\mu_{m+1}}{\mu_{0}}\right| .
$$

Proposition 5.1. The integral

$$
\int_{X} \bar{r}_{m}\left(\mu_{0}: \ldots: \mu_{m+1}\right)
$$

satisfies the following properties:

(1) Skew symmetry with respect to the permutations of $\mu_{i}$.

(2) Homogeneity:

$$
\int_{X} \bar{r}_{m}\left(\lambda_{0} \mu_{0}: \ldots: \lambda_{m+1} \mu_{m+1}\right)=\int_{X} \bar{r}_{m}\left(\mu_{0}: \ldots: \mu_{m+1}\right) .
$$

(3)Additivity: for any $m+3$ measures $\mu_{i}$ on $X$ one has

$$
\sum_{i=0}^{m+2}(-1)^{i} \int_{X} \bar{r}_{m}\left(\mu_{0}: \ldots: \widehat{\mu}_{i}: \ldots: \mu_{m+2}\right)=0 .
$$


(4) Let $g$ be a diffeomorphism of $X$. Then

$$
\int_{X} \bar{r}_{m}\left(g^{*} \mu_{0}: \ldots: g^{*} \mu_{m+1}\right)=\int_{X} \bar{r}_{m}\left(\mu_{0}: \ldots: \mu_{m+1}\right) .
$$

Proof. (1). Follows from $\log f \cdot d \log g+\log g \cdot d \log f=d(\log f \cdot \log g)$.

(2). Using (1) we may assume $\lambda_{i}=1$ for $i>0$. Then

$$
\begin{aligned}
& \int_{X}\left(\bar{r}_{m}\left(\lambda_{0} \mu_{0}: \mu_{1}: \ldots: \mu_{m+1}\right)-\bar{r}_{m}\left(\mu_{0}: \mu_{1}: \ldots: \mu_{m+1}\right)\right) \\
= & -\log |\lambda| \cdot \int_{X} d\left(\log \left|\frac{\mu_{2}}{\mu_{0}}\right| d \log \left|\frac{\mu_{3}}{\mu_{0}}\right| \wedge \ldots \wedge d \log \left|\frac{\mu_{m+1}}{\mu_{0}}\right|\right)=0 .
\end{aligned}
$$

(3) Taking into account the skewsymmetry of the integral we have to prove that

$$
\operatorname{Alt}_{(0, \ldots, m+2)}\left\{\log \left|\frac{\mu_{2}}{\mu_{1}}\right| d \log \left|\frac{\mu_{3}}{\mu_{1}}\right| \wedge \ldots \wedge d \log \left|\frac{\mu_{m+2}}{\mu_{1}}\right|\right\}=0 .
$$

Let us write $\frac{\mu_{i}}{\mu_{j}}=\frac{\mu_{i}}{\mu_{0}} / \frac{\mu_{j}}{\mu_{0}}$ and substitute it into (54). Then the terms in (54) where

$$
\log \left|\frac{\mu_{2}}{\mu_{0}}\right| d \log \left|\frac{\mu_{3}}{\mu_{0}}\right| \wedge \ldots \wedge d \log \left|\frac{\mu_{m+2}}{\mu_{0}}\right|
$$

will appear to look as follows:

$$
\begin{aligned}
\log \left|\frac{\mu_{2}}{\mu_{1}}\right| d \log \left|\frac{\mu_{3}}{\mu_{1}}\right| \wedge \ldots \wedge d \log \left|\frac{\mu_{m+2}}{\mu_{1}}\right|-\log \left|\frac{\mu_{1}}{\mu_{2}}\right| d \log \left|\frac{\mu_{3}}{\mu_{2}}\right| \wedge \ldots \wedge d \log \left|\frac{\mu_{m+2}}{\mu_{2}}\right| \\
-\log \left|\frac{\mu_{2}}{\mu_{0}}\right| d \log \left|\frac{\mu_{3}}{\mu_{0}}\right| \wedge \ldots \wedge d \log \left|\frac{\mu_{m+2}}{\mu_{0}}\right|+\log \left|\frac{\mu_{0}}{\mu_{2}}\right| d \log \left|\frac{\mu_{3}}{\mu_{2}}\right| \wedge \ldots \wedge d \log \left|\frac{\mu_{m+2}}{\mu_{2}}\right| .
\end{aligned}
$$

(The first two terms come from $\operatorname{Alt}_{(1, \ldots, m+2)} \bar{r}_{m}\left(\mu_{1}: \ldots: \mu_{m+2}\right)$ and the second two from $\operatorname{Alt}_{(0,2, \ldots, m+2)} \bar{r}_{m}\left(\mu_{0}: \mu_{2}: \ldots: \mu_{m+2}\right)$. The expression $\operatorname{Alt}_{m+2} \bar{r}_{m}\left(\mu_{0}: \ldots: \widehat{\mu}_{i}:\right.$ $\ldots: \mu_{m+2}$ ) provides no such terms if $i>1$.)

(4) Clear. The proposition is proved.

Recall the following general construction. Let $G$ be a group. Let $X$ be a $G$-set and $f$ a function on $X^{n}$ satisfying

$$
\sum_{i=1}^{n+1}(-1)^{i} f\left(x_{1}, \ldots, \widehat{x}_{i}, \ldots, x_{n+1}\right)=0 .
$$

Choose a point $x \in X$. Then there is an $(n-1)$-cocycle of the group $G$ :

$$
f_{x}\left(g_{1}, \ldots, g_{n}\right):=f\left(g_{1} x, \ldots, g_{n} x\right) .
$$

Lemma 5.2. The cohomology class of the cocycle $f_{x}$ does not depend on $x$.

Proof. The difference $f_{y}-f_{x}$ is the coboundary of the $(n-2)$-chain

$$
h_{x, y}\left(g_{1}, \ldots, g_{n-1}\right)=\sum_{i=1}^{n-1}(-1)^{k-1} f\left(g_{1} x, g_{2} x, \ldots, g_{k} x, g_{k} y, g_{k+1} y, \ldots, g_{n-1} y\right) .
$$

Here is the geometric picture leading to this formula. Consider the prism $\Delta_{g_{1}, \ldots, g_{n}}^{(n-1)} \times$ $\Delta_{x, y}^{(1)}$ given by the product of the $(n-1)$-simplex with vertices $g_{1}, \ldots, g_{n}$ by the 1 simplex with vertices $(x, y)$. Decomposing its side face $\Delta_{g_{1}, \ldots, g_{n-1}}^{(n-2)} \times \Delta_{x, y}^{(1)}$ into simplices we come to the right-hand side of (55). Then the terms of the formula $f_{y}-f_{x}-\delta h_{x, y}$ correspond to the boundary faces of the prism. Cutting the prism into simplices we see that the sum of the terms corresponding to the prism boundary is zero thanks to the cocycle relation. The lemma is proved. 
So, for any $x \in \mathbb{H}_{n}$,

$$
\left(\psi_{n}\right)_{x}\left(g_{0}, \ldots, g_{2 n-1}\right):=\psi_{n}\left(g_{0} x, \ldots, g_{2 n-1} x\right)
$$

is a smooth $(2 n-1)$-cocycle of $G L_{n}(\mathbb{C})$.

Remark. This cocycle is the restriction to $G L_{n}(\mathbb{C})$ of the Bott cocycle for the group of diffeomorphisms of $\mathbb{C P}^{n-1}$.

Let $h_{0}, \ldots, h_{2 n-1}$ be any hyperplanes in $\mathbb{C P}^{n-1}$. Recall that the Grassmannian $n$-logarithm is defined by

$$
\mathcal{L}_{n}^{G}\left(h_{0}, \ldots, h_{2 n-1}\right)=(2 \pi i)^{1-n} \int_{\mathbb{C P}^{n-1}} r_{2 n-1}\left(f_{1}, \ldots, f_{2 n-1}\right)
$$

where $f_{i}$ is a rational function on $\mathbb{C P}^{n-1}$ with the divisor $\left(h_{i}\right)-\left(h_{0}\right)$.

Proposition 5.3. One has

$$
\mathcal{L}_{n}^{\mathrm{G}}\left(h_{0}, \ldots, h_{2 n-1}\right)=-\frac{(-4)^{n-1}(n-1) !^{2}}{(2 \pi i)^{n-1}(2 n-2) !} \int_{\mathbb{C P}^{n-1}} \log \left|f_{1}\right| \bigwedge_{j=2}^{2 n-1} d \log \left|f_{j}\right| .
$$

Proof. See Proposition 6.2 in [GZ].

3. The Grassmannian polylogarithm $\mathcal{L}_{n}^{G}$ as the boundary value of the function $\psi_{n}$. We start from an explicit formula for the Fubini-Studi form. Let $\widehat{\mathbb{P}}^{n-1}$ be the variety of all hyperplanes in $\mathbb{P}^{n-1}$. Consider the incidence divisor

$$
D \subset \widehat{\mathbb{P}}^{n-1} \times \mathbb{P}^{n-1}, \quad D:=\{(h, x) \mid x \in h\}
$$

where $h$ is a hyperplane and $x$ is a point in $\mathbb{P}^{n-1}$.

Let $\left(x_{0}: \ldots: x_{n-1}\right)$ be the homogeneous coordinates of a point $x$ in $\mathbb{P}^{n-1}$. Let

$$
\sigma_{n}(x, d x):=\sum_{i=0}^{n-1}(-1)^{i} x_{i} d x_{0} \wedge \ldots \wedge \widehat{d x_{i}} \wedge \ldots \wedge d x_{n-1}=i_{E} \operatorname{vol}_{x}
$$

be the Leray form. Here $\operatorname{vol}_{x}=d x_{0} \wedge \ldots \wedge d x_{n-1}$ and $E=\sum x_{i} \partial_{x_{i}}$.

There is a canonical differential $(n-1, n-1)$-form $\omega_{D}$ on $\widehat{\mathbb{P}}^{n-1} \times \mathbb{P}^{n-1}-D$ with a polar singularity at the divisor $D$. Namely, let $x \in V_{n}$ and $\xi \in V_{n}^{*}$. Then

$$
\omega_{D}:=\frac{1}{(2 \pi i)^{n-1}} \frac{\sigma_{n}(\xi, d \xi) \wedge \sigma_{n}(x, d x)}{\langle\xi, x\rangle^{n}} .
$$

It is $P G L_{n}$-invariant. A Hermitian metric $H$ in $V_{n}$ provides an isomorphism $H$ :

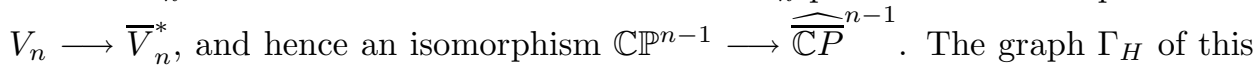
map does not intersect the incidence divisor $D$. Thus restricting the form $\omega_{D}$ to $\Gamma_{H}$ we get a volume form on $\mathbb{C} P^{n-1}$ :

$$
\omega_{F S}(H):=\frac{1}{(2 \pi i)^{n-1}} \frac{\sigma_{n}(z, d z) \wedge \sigma_{n}(\bar{z}, d \bar{z})}{H(z, \bar{z})^{n}} .
$$

It is clearly invariant under the group preserving the Hermitian form $H$. Moreover, it is the Fubini-Studi volume form: a proof can be obtained by using the explicit formula for the Fubini-Studi Kähler form given in [A], complement 3.

One can realize $\mathbb{C P}^{n-1}$ as the smallest stratum of the boundary of $\mathbb{H}_{n}$. Namely, for a hyperplane $h$ in an $n$-dimensional complex vector space $V_{n}$ let

$$
F_{h}:=\left\{\text { nonnegative definite Hermitian forms in } V_{n} \text { with kernel } h\right\} / \mathbb{R}_{+}^{*} .
$$


The set of Hermitian forms in $V_{n}$ with the kernel $h$ is isomorphic to $\mathbb{R}_{+}^{*}$, so $F_{h}$ defines a point on the boundary of $\overline{\mathbb{H}}_{n}$.

For any nonzero nonnegative definite Hermitian form $H$ one can define the corresponding Fubini-Studi form by formula (56). It is a differential form with singularities along the projectivization of the kernel of $H$. In particular, if $h$ is a hyperplane, then the degenerate Hermitian form $F_{h}$ provides the Lebesgue measure on the affine space $\mathbb{C P}^{n-1}-h$. Indeed, if $h_{0}=\left\{z_{0}=0\right\}$, then (56) specializes to

$$
\frac{1}{(2 \pi i)^{n-1}} d \frac{z_{1}}{z_{0}} \wedge \ldots \wedge d \frac{z_{n-1}}{z_{0}} \wedge d \frac{\bar{z}_{1}}{\bar{z}_{0}} \wedge \ldots \wedge d \frac{\bar{z}_{n-1}}{\bar{z}_{0}} .
$$

Denote by $M_{h}$ the one-dimensional real vector space generated by this form. For any hyperplane $h$ in $\mathbb{C P}^{n-1}$ let us choose a measure $\mu_{h} \in M_{h}$.

Proposition 5.4. For any $2 n$ hyperplanes $h_{0}, \ldots, h_{2 n-1}$ in $\mathbb{C P}^{n-1}$ the integral

$$
\psi_{n}\left(h_{0}, \ldots, h_{2 n-1}\right):=\int_{\mathbb{C P}^{n-1}} \log \left|\frac{\mu_{h_{1}}}{\mu_{h_{0}}}\right| d \log \left|\frac{\mu_{h_{2}}}{\mu_{h_{0}}}\right| \wedge \ldots \wedge d \log \left|\frac{\mu_{h_{2 n-1}}}{\mu_{h_{0}}}\right|
$$

is convergent and equal to

$$
(-4)^{-n} \cdot(2 \pi i)^{n-1}(2 n)^{2 n-1}\left(\begin{array}{c}
2 n-2 \\
n-1
\end{array}\right) \cdot \mathcal{L}_{n}^{G}\left(h_{0}, \ldots, h_{2 n-1}\right) .
$$

Proof. Let $h_{1}, h_{2}$ be hyperplanes in $\mathbb{C P}^{n-1}$ and $f$ be a rational function such that $(f)=\left(h_{1}\right)-\left(h_{2}\right)$. From the explicit description of $M_{h}$ given above we immediately see that

$$
\mu_{h_{1}} / \mu_{h_{2}}=\lambda \cdot|f|^{2 n} .
$$

Using this and Theorem 2.4 we see that integral (57) is convergent. The second statement follows from Proposition [5.3 and (58). The proposition is proved.

More generally, take any $2 n$ Hermitian forms $H_{0}, \ldots, H_{2 n-1}$, possibly degenerate. For each of the forms $H_{i}$ consider the corresponding measure $\mu_{H_{i}}$ (a multiple of the Fubini-Studi form related to $H_{i}$ ). Using the convergence of the integral (57) we can deduce that the integral

$$
\begin{aligned}
& \psi_{n}\left(H_{0}, \ldots, H_{2 n-1}\right):=\int_{\mathbb{C P}^{n-1}} \log \left|\frac{\mu_{H_{1}}}{\mu_{H_{0}}}\right| d \log \left|\frac{\mu_{H_{2}}}{\mu_{H_{0}}}\right| \wedge \ldots \wedge d \log \left|\frac{\mu_{H_{2 n-1}}}{\mu_{H_{0}}}\right| \\
= & -n^{2 n-1} \cdot \int_{\mathbb{C P}^{n-1}} \log \left|\frac{H_{1}(z, \bar{z})}{H_{0}(z, \bar{z})}\right| d \log \left|\frac{H_{2}(z, \bar{z})}{H_{0}(z, \bar{z})}\right| \wedge \ldots \wedge d \log \left|\frac{H_{2 n-1}(z, \bar{z})}{H_{0}(z, \bar{z})}\right|
\end{aligned}
$$

is also convergent. This enables us to extend $\psi_{n}$ to the function $\bar{\psi}_{n}\left(x_{0}, \ldots, x_{2 n-1}\right)$ on the configuration space of $2 n$ points in $\overline{\mathbb{H}}_{n-1}$. The function $\bar{\psi}_{n}$ is discontinuous. For instance it is discontinuous at the point $x_{1}=\ldots=x_{2 n-1}=F_{h}$ for a given hyperplane $h$ in $\mathbb{C P}^{n-1}$. It is however a smooth function on an open part of any given strata. We will keep the notation

$$
\psi_{n}\left(h_{0}, \ldots, h_{2 n-1}\right)=\bar{\psi}_{n}\left(F_{h_{0}}, \ldots, F_{h_{2 n-1}}\right) .
$$

Applying Lemma 5.2 to the case when $X$ is $\overline{\mathbb{H}}_{n}$ and using only the fact that the function $\bar{\psi}_{n}\left(x_{0}, \ldots, x_{2 n-1}\right)$ is well-defined for any $2 n$ points in $\overline{\mathbb{H}}_{n}$ and satisfies the cocycle condition for any $2 n+1$ of them we get 
Corollary 5.5. Let $x \in \mathbb{H}_{n}$ and let $h$ be a hyperplane in $\mathbb{C P}^{n-1}$. Then the cohomology classes of the following cocycles coincide:

$$
\psi_{n}\left(g_{0} x, \ldots, g_{2 n-1} x\right) \text { and } \psi_{n}\left(g_{0} h, \ldots, g_{2 n-1} h\right) .
$$

4. A normalization of the Borel class $b_{n}$. Choose a Hermitian metric in $V_{n}$. Let $e$ be the corresponding point of the symmetric space $\mathbb{H}_{n}$; its stabilizer is the subgroup $S U(n)$. One has

$$
\left(\Lambda^{\bullet} T_{e}^{*} \mathbb{H}_{n}\right)^{S U(n)}=\mathcal{A}^{\bullet}\left(S L_{n}(\mathbb{C}) / S U(n)\right)^{S L_{n}(\mathbb{C})} .
$$

There are well-known canonical ring isomorphisms (see [B2] and the references there):

$$
\begin{gathered}
\left(\Lambda^{\bullet} T_{e}^{*} \mathbb{H}_{n}\right)^{S U(n)} \otimes_{\mathbb{R}} \mathbb{C}=\Lambda^{\bullet}\left(s l_{n}(\mathbb{C})\right)^{s l_{n}(\mathbb{C})} \\
=H^{\bullet}\left(s l_{n}(\mathbb{C}), \mathbb{C}\right) \stackrel{\alpha}{=} H_{\text {top }}^{\bullet}(S U(n), \mathbb{C}) \stackrel{\beta}{=} H_{m}^{\bullet}\left(S L_{n}(\mathbb{C}), \mathbb{C}\right)
\end{gathered}
$$

where $H^{\bullet}$ is the Lie algebra cohomology, $H_{\text {top }}^{\bullet}$ is the topological cohomology, and $H_{m}(G)$ denotes the measurable cohomology of a Lie group $G$. The first isomorphism is obvious: $T_{e} \mathbb{H}_{n} \otimes_{\mathbb{R}} \mathbb{C}=s l_{n}(\mathbb{C})$. The map

$$
\alpha_{\mathrm{DR}}: \Lambda_{\mathbb{Q}}^{\bullet}\left(s l_{n}\right)^{s l_{n}} \stackrel{\sim}{\longrightarrow} H_{\mathrm{DR}}^{\bullet}\left(S L_{n}(\mathbb{C}), \mathbb{Q}\right)
$$

sends an $s l_{n}$-invariant exterior form on $s l_{n}$ to the right-invariant one, and hence to the bi-invariant differential form on $S L_{n}(\mathbb{C})$. Let us describe the map

$$
\beta_{\mathrm{DR}}: H_{\mathrm{DR}}^{\bullet}\left(S L_{n}(\mathbb{C}), \mathbb{Q}\right) \longrightarrow H_{m}^{\bullet}\left(S L_{n}(\mathbb{C}), \mathbb{C}\right) .
$$

Let $C$ be a bi-invariant, and hence closed, differential $(2 n-1)$-form on $S L_{n}(\mathbb{C})$. Let us restrict it first to the Lie algebra, and then to the orthogonal complement $s u(n)^{\perp}$ to the Lie subalgebra $s u(n) \subset s l_{n}(\mathbb{C})$. We identify the $\mathbb{R}$-vector spaces $T_{e} \mathbb{H}_{n}$ and $s u(n)^{\perp}$. The obtained exterior form on $T_{e} \mathbb{H}_{n}$ is the restriction of an invariant differential form, denoted $\omega_{C}$, on the symmetric space $\mathbb{H}_{n}$. It is a closed differential form.

For any ordered $2 n$ points $x_{1}, \ldots, x_{2 n}$ in $\mathbb{H}_{n}$ there is a geodesic simplex $I\left(x_{1}, \ldots\right.$, $\left.x_{2 n}\right)$ in $\mathbb{H}_{n}$. It is constructed inductively as follows. Let $I\left(x_{1}, x_{2}\right)$ be the geodesic from $x_{1}$ to $x_{2}$. The geodesics from $x_{3}$ to the points of $I\left(x_{1}, x_{2}\right)$ form a geodesic triangle $I\left(x_{1}, x_{2}, x_{3}\right)$. All the geodesics from $x_{4}$ to the points of the geodesic triangle $I\left(x_{1}, x_{2}, x_{3}\right)$ form a geodesic simplex $I\left(x_{1}, x_{2}, x_{3}, x_{4}\right)$, and so on. When the rank of the symmetric space is greater than 1 (i.e., $n>2)$ the geodesic simplex $I\left(x_{1}, \ldots, x_{k}\right)$ depends on the ordering of the vertices $x_{1}, \ldots, x_{k}$.

The differential $(2 n-1)$-form $\omega_{C}$ on $S L_{n}(\mathbb{C}) / S U(n)$ provides a volume of the geodesic simplex:

$$
\operatorname{vol}_{C} I\left(x_{1}, \ldots, x_{2 n}\right):=\int_{I\left(x_{1}, \ldots, x_{2 n}\right)} \omega_{C} .
$$

For every $2 n+1$ points $x_{1}, \ldots, x_{2 n+1}$ the boundary of the simplex $I\left(x_{1}, \ldots, x_{2 n+1}\right)$ is the alternating sum of the simplices $I\left(x_{1}, \ldots, \widehat{x}_{i}, \ldots, x_{2 n+1}\right)$. Since the form $\omega_{C}$ is closed, the Stokes theorem yields

$$
\sum_{i=1}^{2 n+1}(-1)^{i} \int_{I\left(x_{1}, \ldots, \widehat{x}_{i}, \ldots, x_{2 n+1}\right)} \omega_{C}=\int_{I\left(x_{1}, \ldots, x_{2 n+1}\right)} d \omega_{C}=0 .
$$


This just means that for a given point $x$ the function $\operatorname{vol}_{\mathrm{C}} I\left(g_{1} x, \ldots, g_{2 n} x\right)$ is a smooth $(2 n-1)$-cocycle of the Lie group $S L_{n}(\mathbb{C})$. It was considered by J. Dupont D. By Lemma 5.2 cocycles corresponding to different points $x$ are canonically cohomologous. The obtained cohomology class is the class $\beta_{\mathrm{DR}}([\mathrm{C}])$.

Remark. $\operatorname{vol}_{C} I\left(x_{1}, \ldots, x_{2 n}\right)$ is independent up to a sign of the ordering of its vertices. Indeed, consider $2 n+1$ points $\left(x_{1}, x_{2}, x_{1}, x_{3}, \ldots, x_{2 n}\right)$ and apply relation (61).

The Betti cohomology of $S L_{n}(\mathbb{C})$. Recall that $S U(n)$ is a retract of $S L_{n}(\mathbb{C})$. It is well-known that

$$
H_{\text {top }}^{\bullet}(S U(n), \mathbb{Z})=H_{\text {top }}^{\bullet}\left(S^{3} \times S^{5} \times \ldots \times S^{2 n-1}, \mathbb{Z}\right)=\Lambda^{*}\left(B_{3}, B_{5}, \ldots, B_{2 n-1}\right) .
$$

The restriction from $S U(n)$ to $S U(m)$ kills the classes $B_{2 k-1}$ for $k>m$. If $k \leq m$, it identifies the class $B_{2 k-1}$ for $S U(n)$ with the one for $S U(m)$. The class $B_{2 n-1}$ for $S U(n)$ is provided by the fundamental class of the sphere $S^{2 n-1} \subset \mathbb{C}^{n}$. Namely, it is the pull-back of the fundamental class under the map $S U(n) \longrightarrow S^{2 n-1}$ provided by a choice of a point on $S^{2 n-1}$. This sphere has the orientation induced by that of $\mathbb{C}^{n}$. Thus

$$
\mathbb{Z} \cdot B_{n}=\operatorname{Ker}\left(H_{\text {top }}^{2 n-1}(S U(n), \mathbb{Z}) \longrightarrow H_{\text {top }}^{2 n-1}(S U(n-1), \mathbb{Z})\right) .
$$

The transgression in the Leray spectral sequence for the universal $S U(n)$-bundle $E U(n) \longrightarrow B U(n)$ provides an isomorphism

$$
\mathbb{Z} \cdot B_{n} \longrightarrow \frac{H^{2 n}(B S U(n), \mathbb{Z})}{\oplus_{0<i<2 n} H^{i} \cdot H^{2 n-i}}
$$

and identifies $B_{n}$ with the Chern class $c_{n} \in H^{2 n}(B S U(n), \mathbb{Z})$ of the associated vector bundle.

The de Rham cohomology of $S L_{n}(\mathbb{C})$. Consider the differential form

$$
\omega_{D_{n}}:=\operatorname{tr}\left(g^{-1} d g\right)^{2 n-1} \in \Omega^{2 n-1}\left(S L_{N}\right) .
$$

Its restriction to the subgroup $S L_{m}$ is zero for $m<n$. It follows that the cohomology class

$$
\left[\omega_{D_{n}}\right] \in H_{\mathrm{DR}}^{2 n-1}\left(S L_{n}, \mathbb{C}\right)
$$

is a multiple of $B_{n}$. The Hodge considerations show that $\left[\omega_{D_{n}}\right] \in(2 \pi i)^{n} \mathbb{Q} \cdot B_{n}$.

Lemma 5.6. The differential form $\omega_{D_{n}}$ is an $\mathbb{R}(n-1)$-valued form. In particular, it provides a cohomology class

$$
b_{n}:=\beta_{\mathrm{DR}}\left(\omega_{D_{n}}\right) \in H_{m}^{2 n-1}\left(S L_{n}(\mathbb{C}), \mathbb{R}(n-1)\right) .
$$

Proof. An easy calculation shows that the value of the exterior form $\omega_{D_{n} \mid T_{e} \mathbb{H}_{n}}$ on

$$
\left(e_{1, n}+e_{n, 1}\right) \wedge i\left(e_{1, n}-e_{n, 1}\right) \wedge \ldots \wedge\left(e_{n-1, n}+e_{n, n-1}\right) \wedge i\left(e_{n-1, n}-e_{n, n-1}\right) \wedge e_{n, n}
$$

is nonzero and obviously lies in $\mathbb{Q}(n-1)$.

On the other hand, the values of the form $\omega_{D_{n}}$ lie in a one-dimensional $\mathbb{R}$-vector space. Indeed, the space of $s u(n)$-invariant real exterior $(2 n-1)$-forms on the space of all Hermitian $n \times n$ matrices, which have zero restriction to the subspace of Hermitian $(n-1) \times(n-1)$ matrices, is one-dimensional. The exterior form $\omega_{D_{n} \mid T_{e} \mathbb{H}_{n}}$ belongs to the complexification of this space. The lemma follows from this.

We call the cohomology class provided by this lemma the Borel class and use it below to construct the Borel regulator. 
5. Comparison of the Grassmannian and Borel cohomology classes of $G L_{n}(\mathbb{C})$. Let $\left[C_{n}^{G}\right]$ be the cohomology class of the $(2 n-1)$-cocycle of $G L_{n}(\mathbb{C})$ provided by the Grassmannian $n$-logarithm (see Corollary 5.5). We want to compare it with the Borel class.

Let us consider the following integral:

$$
:=-n^{2 n-1} \cdot \int_{\mathbb{C P}^{n-1}} \frac{\widetilde{C}_{n}\left(H_{1}, \ldots, H_{2 n-1}\right)}{(z, \bar{z})} d \frac{H_{2}(z, \bar{z})}{(z, \bar{z})} \wedge \ldots \wedge d \frac{H_{2 n-1}(z, \bar{z})}{(z, \bar{z})}
$$

where the $H_{i}$ are arbitrary complex matrices and $H_{i}(z, \bar{z})$ are the bilinear forms in $z, \bar{z}$ given by the matrix $H_{i}$. We claim that it is a $(2 n-1)$-cocycle of the Lie algebra $g l_{n}(\mathbb{C})$ and it is obtained by differentiating the group cocycle provided by the function (59). We put these facts in the following framework.

If we restrict to the case when the $H_{i}$ are Hermitian matrices, integral (64) admits the following interpretation. Let us construct a map

$$
\mathbb{M}_{e}: \mathbb{C P}^{n-1} \longrightarrow T_{e}^{*} \mathbb{H}_{n}
$$

which is a version of the moment map. For a point $z \in \mathbb{C P}^{n-1}$ the value of the $\left\langle M_{e}(z), v\right\rangle$ of the functional $\mathbb{M}_{e}(z)$ on a vector $v \in T_{e} \mathbb{H}_{n}$ is defined as follows. Let $e(t)$ be a path in $\mathbb{H}_{n}$ such that $e(0)=e$ and $\dot{e}(0)=v$. Recall the measure $\mu_{x}$ defined in Subsection 1 in Section 5. Then

$$
\left\langle\mathbb{M}_{e}(z), v\right\rangle:=\left.\frac{d}{d t} \log \frac{\mu_{e(t)}(z)}{\mu_{e}(z)}\right|_{t=0} .
$$

Choose coordinates $z_{1}, \ldots, z_{n}$ in $V_{n}$ such that $(z, \bar{z}):=\left|z_{1}\right|^{2}+\ldots+\left|z_{n}\right|^{2}$ corresponds to the point $e$. Then $T_{e} \mathbb{H}_{n}$ is identified with the space of Hermitian $(n \times n)$ matrices $H$. It follows from (56) that

$$
\left\langle\mathbb{M}_{e}(z), H\right\rangle:=n \frac{H(z, \bar{z})}{(z, \bar{z})} .
$$

The map $\mathbb{M}_{e}$ is clearly $S U(n)$-invariant. Its image is an $S U(n)$-orbit in $T_{e}^{*} \mathbb{H}_{n}$ isomorphic to $\mathbb{C P}^{n-1}$.

We need the following general construction. Let $V$ be a real vector space and $M$ a compact subset of $V^{*}$ which is the closure of a $k$-dimensional submanifold. Any element $\omega \in \Lambda^{k} V$ can be viewed as a $k$-form $\omega$ on $V^{*}$. Integrating it over $M$ we get an exterior form $C_{M} \in \Lambda^{k} V^{*}$. If $M$ is a cone over $M^{\prime}$ with the vertex at the origin, then $\int_{M} \omega=\int_{M^{\prime}} i_{E} \omega$ where $E$ is the Euler vector field on $V$.

Applying this construction to the cone over the orbit $M_{e}$ based at the origin we get an $S U(n)$-invariant element $\widetilde{C}_{n} \in \Lambda^{2 n-1} T_{e}^{*} \mathbb{H}_{n}$. It follows from 65) that it is given by formula (64) multiplied by $2 n$.

Another invariant $(2 n-1)$-cocycle $C_{n}$ of the Lie algebra $g l_{n}$, considered by Dynkin [Dyn, is given by

$$
C_{n}\left(X_{1}, \ldots, X_{2 n-1}\right)=\frac{1}{n !} \operatorname{Alt}_{2 n-1} \operatorname{Tr}\left(X_{1} X_{2} \ldots X_{2 n-1}\right) .
$$

Let $\left[C_{n}\right]$ be the cohomology class of $G L_{n}(\mathbb{C})$ corresponding to the cocycle $C_{n}$.

Theorem 5.7. One has

$$
\widetilde{C}_{n}=-(-1)^{\frac{(n-1) n}{2}} \frac{(2 \pi i)^{n-1} n^{2 n-1}(n-1) !}{(2 n-1) !} \cdot C_{n}
$$


and the class $\left[C_{n}^{G}\right]$ is a nonzero rational multiple of $\left[C_{n}\right]$ :

$$
\left[C_{n}^{G}\right]=-(-1)^{\frac{n(n+1)}{2}} \frac{(n-1) !^{3}}{(2 n-2) !(2 n-1) !}\left[C_{n}\right] .
$$

Proof. The second claim follows from the first using Proposition 5.4 .

Let us prove the first claim. The restriction of the cocycle $C_{n}$ to the Lie subalgebra $g l_{n-1}(\mathbb{C})$ equals zero. This follows, for instance, from the Amitsur-Levitsky theorem: for any $n \times n$ matrices $A_{1}, \ldots, A_{2 n}$ one has $\operatorname{Alt}_{2 n}\left(A_{1}, \ldots, A_{2 n}\right)=0$.

On the other hand, the restriction of the cocycle $\widetilde{C}_{n}$ to the Lie subalgebra of matrices $\left(a_{i j}\right)$ where $a_{1 j}=a_{j 1}=0$ is zero. Indeed, in this case the form we integrate in (64) is a differential $(2 n-2)$-form in $d z_{2}, \ldots, d z_{n-1}, d \bar{z}_{2}, \ldots, d \bar{z}_{n-1}$ and thus it is zero. So thanks to (60) and (62) we conclude that the cocycle $C_{n}$ is proportional to $\widetilde{C}_{n}$. To determine the proportionality coefficient we compute the values of both cocycles on a special element $E_{n} \in \Lambda^{2 n-1} g l_{n}$. To write it down denote by $e_{i, j}$ the elementary $n \times n$ matrix whose only nonzero entry is 1 in the $(i, j)$ place. Then

$$
E_{n}:=\bigwedge_{j=1}^{n-1}\left(e_{j, n} \wedge e_{n, j}\right) \wedge e_{n, n} .
$$

A direct computation shows that

$$
\left\langle C_{n}, E_{n}\right\rangle=1 .
$$

Indeed, to get a nonzero trace we have to multiply $(n-1)$ blocks $e_{n, j} e_{j, n}$, as well as $e_{n, n}$, which can be inserted anywhere between these blocks. So there are $(n-1) ! n=n$ ! possibilities.

Let us compute the value of the cocycle $\widetilde{C}_{n}$ on $E_{n}$.

Lemma 5.8. Integral (64) equals

$$
\frac{-n^{2 n-1}}{(2 n-1) !} \cdot \operatorname{Alt}_{2 n-1} \int_{\mathbb{C P}^{n-1}} \frac{H_{1}(z, \bar{z}) d H_{2}(z, \bar{z}) \wedge \ldots \wedge d H_{2 n-1}(z, \bar{z})}{(z, \bar{z})^{2 n-1}} .
$$

Proof. By Proposition 5.1 integral (64) equals

$$
\frac{-n^{2 n-1}}{(2 n-1) !} \operatorname{Alt}_{2 n-1} \cdot \int_{\mathbb{C P}^{n-1}} \frac{H_{1}(z, \bar{z})}{(z, \bar{z})} d \frac{H_{2}(z, \bar{z})}{(z, \bar{z})} \wedge \ldots \wedge d \frac{H_{2 n-1}(z, \bar{z})}{(z, \bar{z})} .
$$

One has, for $i=2, \ldots, 2 n-1$, that

$$
d \frac{H_{i}(z, \bar{z})}{(z, \bar{z})}=\frac{(z, \bar{z}) d H_{i}(z, \bar{z})-H_{i}(z, \bar{z}) d(z, \bar{z})}{(z, \bar{z})^{2}} .
$$

Substituting

$$
\frac{-H_{i}(z, \bar{z}) d(z, \bar{z})}{(z, \bar{z})^{2}} \quad \text { instead of } d \frac{H_{i}(z, \bar{z})}{(z, \bar{z})} \text { in (69) }
$$

we get zero since $H_{1}$ and $H_{i}$ appear in a symmetric way and thus disappear after the alternation. The lemma follows.

Let us calculate integral (69) in the special case

$$
H_{2 n-1}(z, \bar{z})=\left|z_{n}\right|^{2}, \quad H_{2 k-1}(z, \bar{z})=z_{k} \bar{z}_{n}, \quad H_{2 k}(z, \bar{z})=z_{n} \bar{z}_{k}
$$


so that $H_{1} \wedge \ldots \wedge H_{2 n-1}=E_{n}$. We will restrict the integrand to the affine part $\left\{z_{n}=1\right\}$ and then perform the integration. Since $d H_{2 n-1}(z, \bar{z})=0$ on $\left\{z_{n}=1\right\}$ and $d z_{k} \wedge d \bar{z}_{k}=-2 i d x_{k} \wedge d y_{k}$ we get

$$
\begin{gathered}
-(-1)^{\frac{(n-1)(n-2)}{2}} \frac{(-2 i)^{n-1} n^{2 n-1}}{2 n-1} \int_{\mathbb{C}^{n-1}} \frac{d^{n-1} x d^{n-1} y}{\left(1+\left|z_{1}\right|^{2}+\ldots+\left|z_{n-1}\right|^{2}\right)^{2 n-1}} \\
=-(-1)^{\frac{(n-1) n}{2}} \frac{(2 i)^{n-1} n^{2 n-1}}{2 n-1} \operatorname{vol}\left(S^{2 n-3}\right) \int_{0}^{\infty} \frac{r^{2 n-3} d r}{\left(1+r^{2}\right)^{2 n-1}} \\
=-(-1)^{\frac{(n-1) n}{2}} \frac{(2 \pi i)^{n-1} n^{2 n-1}}{(n-1) !(2 n-1)}\left(\begin{array}{c}
2 n-2 \\
n-1
\end{array}\right)^{-1}
\end{gathered}
$$

since the volume of the sphere $S^{2 n-3}$ is $\frac{2 \pi^{n-1}}{(n-2) !}$ and

$$
\int_{0}^{\infty} \frac{r^{2 n-3} d r}{\left(1+r^{2}\right)^{2 n-1}}=\frac{1}{2} \int_{0}^{\infty} \frac{r^{n-2} d r}{(1+r)^{2 n-1}}=\frac{1}{2 n-2}\left(\begin{array}{c}
2 n-2 \\
n-1
\end{array}\right)^{-1} .
$$

To get the last equality we integrate by parts:

$$
\begin{gathered}
\int_{0}^{\infty} \frac{r^{n-2} d r}{(1+r)^{2 n-1}}=-\frac{1}{2 n-2} \int_{0}^{\infty} r^{n-2}\left(\frac{1}{(1+r)^{2 n-2}}\right)^{\prime} d r \\
=\frac{n-2}{2 n-2} \int_{0}^{\infty} r^{n-3} \frac{d r}{(1+r)^{2 n-2}} \\
=\ldots=\frac{(n-2) ! n !}{(2 n-2) !} \int_{0}^{\infty} \frac{d r}{(1+r)^{n+1}}=\frac{1}{n-1}\left(\begin{array}{c}
2 n-2 \\
n-1
\end{array}\right)^{-1} .
\end{gathered}
$$

Theorem 5.7 is proved.

6. Construction of the Borel regulator via Grassmannian polylogarithms. Let $G$ be a group. The diagonal map $\Delta: G \longrightarrow G \times G$ provides a homomorphism $\Delta_{*}: H_{n}(G) \longrightarrow H_{n}(G \times G)$. Recall that

$$
\operatorname{Prim} H_{n}(G):=\left\{x \in H_{n}(G) \mid \Delta_{*}(x)=x \otimes 1+1 \otimes x\right\} .
$$

Set $A_{\mathbb{Q}}:=A \otimes \mathbb{Q}$. One has

$$
K_{n}(F)_{\mathbb{Q}}=\operatorname{Prim}_{n}(G L(F))_{\mathbb{Q}}=\operatorname{Prim} H_{n} G L_{n}(F)_{\mathbb{Q}}
$$

where the second isomorphism is provided by Suslin's stabilization theorem.

The Borel regulator is a map

$$
r_{n}^{\mathrm{Bo}}: K_{2 n-1}(\mathbb{C})_{\mathbb{Q}} \longrightarrow \mathbb{R}(n-1)
$$

provided by pairing the class $b_{n} \in H^{2 n-1}\left(G L_{2 n-1}(\mathbb{C}), \mathbb{R}(n-1)\right)$ with the subspace $K_{2 n-1}(\mathbb{C})_{\mathbb{Q}} \subset H_{2 n-1}\left(G L_{2 n-1}(\mathbb{C}), \mathbb{Q}\right)$.

Recall the Grassmannian complex $C_{*}(n)$

$$
\ldots \stackrel{d}{\longrightarrow} C_{2 n-1}(n) \stackrel{d}{\longrightarrow} C_{2 n-2}(n) \stackrel{d}{\longrightarrow} \ldots \stackrel{d}{\longrightarrow} C_{0}(n)
$$

where $C_{k}(n)$ is the free abelian group generated by configurations of $k+1$ vectors $\left(l_{0}, \ldots, l_{k}\right)$ in generic position in an $n$-dimensional vector space over a field $F$, and $d$ is given by the standard formula (see Section 3.1 in [G2]). The group $C_{k}(n)$ is in degree $k$. Since it is a homological resolution of the trivial $G L_{n}(F)$-module $\mathbb{Z}$ (see Lemma 3.1 in [G2]), there is a canonical homomorphism

$$
\varphi_{2 n-1}^{n}: H_{2 n-1}\left(G L_{n}(F)\right) \longrightarrow H_{2 n-1}\left(C_{*}(n)\right) .
$$


Thanks to Lemma 5.6 the Grassmannian $n$-logarithm function provides a homomorphism

$$
\mathcal{L}_{n}^{G}: C_{2 n-1}(n) \longrightarrow \mathbb{R}(n-1) ; \quad\left(l_{0}, \ldots, l_{2 n-1}\right) \longmapsto \mathcal{L}_{n}^{G}\left(l_{0}, \ldots, l_{2 n-1}\right) .
$$

Thanks to the first $(2 n+1)$-term functional equation for $\mathcal{L}_{n}^{G}$, see (47), it is zero on the subgroup $d C_{2 n}(n)$. So it induces a homomorphism

$$
\mathcal{L}_{n}^{G}: H_{2 n-1}\left(C_{*}(n)\right) \longrightarrow \mathbb{R}(n-1) .
$$

Lemma 5.9. The composition $\mathcal{L}_{n}^{G} \circ \varphi_{2 n-1}^{n}$ coincides with the class $\left[C_{n}^{G}\right]$.

Proof. Standard; see [G4].

To construct the Borel regulator we extend, as in Section 3.10 of [G2], the class $\left[C_{n}^{G}\right]$ to a class of $G L_{2 n-1}(\mathbb{C})$. Let us recall the key steps.

Let $\mathbb{Z}[S]$ be the free abelian group generated by a set $S$. Let $F$ be a field. Applying the covariant functor $\mathbb{Z} \longmapsto \mathbb{Z}[X(F)]$ to the bi-Grassmannian $\widehat{G}(n)$ (see Subsection 2 in Section 4), and taking the alternating sum of the obtained homomorphisms, we get a bicomplex. Using Lemma 4.1 we see that it looks as follows (G2], Section 3.7):

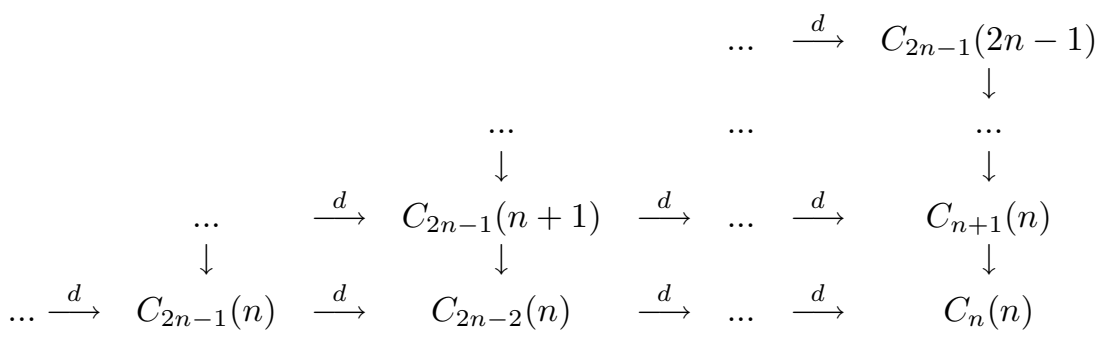

In particular, the bottom row is the stupid truncation of the Grassmannian complex at the group $C_{n}(n)$. The total complex of this bicomplex is a homological complex, called the weight $n$ bi-Grassmannian complex $B C_{*}(n)$. In particular, there is a homomorphism

$$
H_{2 n-1}\left(C_{*}(n)\right) \longrightarrow H_{2 n-1}\left(B C_{*}(n)\right) .
$$

In [G1], G2] we proved that there are homomorphisms

$$
\varphi_{2 n-1}^{m}: H_{2 n-1}\left(G L_{m}(F)\right) \longrightarrow H_{2 n-1}\left(B C_{*}(n)\right), \quad m \geq n
$$

whose restriction to the subgroup $G L_{n}(F)$ coincides with the composition

$$
H_{2 n-1}\left(G L_{n}(F)\right) \stackrel{\varphi_{2 n-1}^{n}}{\longrightarrow} H_{2 n-1}\left(C_{*}(n)\right) \stackrel{\sqrt[72]{\longrightarrow}}{\longrightarrow} H_{2 n-1}\left(B C_{*}(n)\right) \text {. }
$$

Let us extend homomorphism (71) to a homomorphism

$$
\mathcal{L}_{n}^{G}: B C_{2 n-1}(n) \longrightarrow \mathbb{R}(n-1)
$$

by setting it to zero on the groups $C_{2 n-1}(n+i)$ for $i>0$. The second $(2 n-1)$ term functional equation for the Grassmannian $n$-logarithm function, see (48), just means that the composition

$$
C_{2 n}(n+1) \longrightarrow C_{2 n-1}(n) \stackrel{\mathcal{L}_{n}^{G}}{\longrightarrow} \mathbb{R}(n-1),
$$

where the first map is a vertical arrow in $B C_{*}(n)$, is zero. Therefore we get a homomorphism

$$
\mathcal{L}_{n}^{G}: H_{2 n-1}\left(B C_{*}(n)\right) \longrightarrow \mathbb{R}(n-1) .
$$


Corollary 5.10. One has

$$
\left[C_{n}^{G}\right]=-(-1)^{n(n+1) / 2} \frac{(n-1) !^{2}}{(2 n-2) !(2 n-1) !} \cdot \frac{b_{n}}{n}
$$

Proof. Indeed,

$$
\operatorname{Alt}_{2 n-1} \operatorname{Tr}\left(X_{1} \cdot \ldots \cdot X_{2 n-1}\right)=\left\langle\operatorname{tr}\left(g^{-1} d g\right)_{\mid s l_{n}}^{2 n-1}, X_{1} \wedge \ldots \wedge X_{2 n-1}\right\rangle .
$$

So the claim follows from Theorem [5.7] since, as is clear from comparison of formulas (66) and (63), $b_{n}=n !\left[C_{n}\right]$. The corollary is proved.

Theorem 5.11. The composition

$$
\begin{gathered}
K_{2 n-1}(\mathbb{C}) \stackrel{\sim}{\longrightarrow} \operatorname{Prim} H_{2 n-1}\left(G L_{2 n-1}(\mathbb{C}), \mathbb{Q}\right) \stackrel{\varphi_{2 n-1}^{2 n-1}}{\longrightarrow} \\
H_{2 n-1}\left(B C_{*}(n)_{\mathbb{Q}}\right) \stackrel{\mathcal{L}_{n}^{G}}{\longrightarrow} \mathbb{R}(n-1)
\end{gathered}
$$

equals

$$
-(-1)^{n(n+1) / 2} \cdot \frac{(n-1) !^{2}}{n(2 n-2) !(2 n-1) !} r_{n}^{\mathrm{Bo}} .
$$

Proof. Recall that the restriction to $G L_{n}$ of the map $\varphi_{2 n-1}^{2 n-1}$ coincides with the map $\varphi_{2 n-1}^{n}$. Therefore Lemma 5.9 guarantees that the restriction to $G L_{n}(\mathbb{C})$ of the composition of the last two arrows coincides with the map given by the class $\left[C_{2 n-1}^{G}\right]$. So Corollary 5.10 implies the theorem.

7. Comparing $\left[D_{n}\right]$ and $B_{n}$. The following result is not used below.

\section{Theorem 5.12.}

$$
\left[D_{n}\right]=(2 \pi i)^{n}(2 n-1) B_{n} .
$$

Proof. The transgression identifies the class $B_{n}$ with the Chern class of the universal bundle over $B G$, where $G=G L_{n}(\mathbb{C})$. We will compute explicitly the transgression of the $n$-th component of the Chern character of the universal vector bundle $p$ : $E \longrightarrow B G$. Let $\mathcal{A}$ be a connection on $E$. Then the $n$-th Chern class is represented by the $2 n$-form

$$
c_{n}(A):=\frac{\operatorname{tr} F_{\mathcal{A}}^{n}}{(2 \pi i)^{n}}
$$

where $F_{A}:=d \mathcal{A}+\mathcal{A} \wedge \mathcal{A}$ is the curvature form.

Let $q: E G \longrightarrow B G$ be the principal fibration associated with $E$. Then the form $q^{*} c_{n}(\mathcal{A})$ is exact. If $d \omega=q^{*} c_{n}(\mathcal{A})$ and $F$ is a fiber of $q$, then $\left.\omega\right|_{F}$ is closed, its cohomology class is transgressive, and goes to $\left[c_{n}(\mathcal{A})\right]$. To do the computation we choose a connection $\mathcal{A}_{0}$ on $B G$ which is flat in a neighbourhood $U$ of a point $x \in B G$. It provides a trivialization of the bundle $E$ over $U$ as well as a trivialization $\varphi:\left.E G\right|_{U} \longrightarrow G \times U$.

The bundle $q^{*} E$ has a canonical trivialization. It provides a connection $\mathcal{A}_{1}$ on $q^{*} E$. So there are two connections, $q^{*} \mathcal{A}_{0}$ and $\mathcal{A}_{1}$ on $q^{*} E$. One has $\mathcal{A}_{1}=$ $q^{*} \mathcal{A}_{0}+g^{-1} d g$, where $(g, u)=\varphi(x)$. Let

$$
\mathcal{A}(t):=t \mathcal{A}_{1}+(1-t) q^{*} \mathcal{A}_{0}=t g^{-1} d g+q^{*} \mathcal{A}_{0} .
$$

It can be thought of as a connection on the lifting of the bundle $q^{*} E$ to $E G \times[0,1]$; here $t \in[0,1]$. The curvature $F(t)$ of this connection is

$$
F(t)=g^{-1} d g d t+t^{2} g^{-1} d g \wedge g^{-1} d g .
$$


The push-forward of the form $\operatorname{tr} F(t)^{n}$ down to $E G$ is a primitive for the form $\operatorname{tr} F_{\mathcal{A}(1)}^{n}-\operatorname{tr} F_{\mathcal{A}(0)}^{n}$. It is given (in $q^{-1} U$ ) by

$$
\int_{0}^{1} \operatorname{tr} F(t)^{n}=\frac{1}{2 n-1} \operatorname{tr}\left(g^{-1} d g\right)^{2 n-1} .
$$

Theorem 5.12 is proved.

8. On the motivic nature of the Grassmannian $n$-logarithm functions. According to the results of the previous section, understanding of the Borel regulator, and hence special values of the Dedekind $\zeta$-functions, is reduced to the study of properties of the Grassmannian $n$-logarithm function $\mathcal{L}_{n}^{G}$.

Recall that a framed mixed Hodge-Tate structure has a natural $\mathbb{R}$-valued invariant $([\mathrm{BD}])$, called the Lie period. Thus a variation of the Hodge-Tate structures $\mathbb{L}$ over a base $X$ provides a period function $\mathbb{L}^{\mathcal{M}}$ on $X(\mathbb{C})$.

Conjecture 5.13. (a) There exists a variation $\mathbb{L}_{n}^{\mathcal{M}}$ of framed mixed Tate motives over $\widehat{G}_{n-1}^{n}$ such that

$$
\sum_{i=0}^{2 n}(-1)^{i} a_{i}^{*} \mathbb{L}_{n}^{\mathcal{M}}=0 ; \quad \sum_{j=0}^{2 n}(-1)^{j} b_{j}^{*} \mathbb{L}_{n}^{\mathcal{M}}=0 ;
$$

and the Lie period $\mathcal{L}_{n}^{\mathcal{M}}$ of its Hodge realization satisfies

$$
\mathcal{L}_{n}^{\mathcal{M}}-\mathcal{L}_{n}^{G}=\sum_{i=0}^{2 n-1}(-1)^{i} a_{i}^{*} F_{n}
$$

where $F_{n}$ is a function on $\widehat{G}_{n-2}^{n}(\mathbb{C})$.

(b) The functional equations satisfied by $\mathcal{L}_{n}^{\mathcal{M}}$ essentially determine it: the space of all smooth/measurable functions satisfying these functional equations is finite dimensional.

Remark. The function $F_{n}$ is obviously not determined by (75) - add a function coming from $\widehat{G}_{n-3}^{n}(\mathbb{C})$. Nevertheless we expect that there exists a canonical explicit choice for $F_{n}$. Then formula (75) can be considered as an explicit formula for $\mathcal{L}_{n}^{\mathcal{M}}$ in terms of $\mathcal{L}^{G}$ 's.

Moreover we expect that there exists a canonical homotopy between the Grassmannian $n$-logarithm (understood as a cocycle in the Deligne cohomology of the bi-Grassmannian) and its "motivic" bi-Grassmannian counterpart. Observe that the motivic bi-Grassmannian $n$-logarithm should have nontrivial components outside of the bottom line of the bi-Grassmannian, while the defined above (or in [G5]) Grassmannian $n$-logarithm is concentrated entirely at the bottom line.

A variation of mixed Tate motives over $\widehat{G}_{n-1}^{n}$ was constructed in [HM]. However, it is not clear how to relate it to the function $\mathcal{L}_{n}^{G}$.

Conjecture 5.13 is known for $n=2$ and $n=3$.

The $n=2$ case follows from (12), the well-known motivic realization of the dilogarithm, and Bloch's theorem characterizing the Bloch-Wigner function by Abel's 5 -term equation that it satisfies.

The $n=3$ case of conjecture 5.13 follows from the results of G1, G2, GZ and the motivic realization of the trilogarithm. In particular, the part (b) is given by Theorem 1.10 in G1. 
Examples. (1) $n=2$. Then $\mathcal{L}_{2}^{\mathcal{M}}=\mathcal{L}_{2}^{G}$.

(2) $n=3$. The motivic Grassmannian trilogarithm function has been constructed in [G1, G2] in terms of the classical trilogarithm function. Namely, one has

$$
\mathcal{L}_{3}^{\mathcal{M}}\left(l_{0}, \ldots, l_{5}\right)=\frac{1}{90} \operatorname{Alt}_{6} \mathcal{L}_{3}\left(\frac{\Delta\left(l_{0}, l_{1}, l_{3}\right) \Delta\left(l_{1}, l_{2}, l_{4}\right) \Delta\left(l_{2}, l_{0}, l_{5}\right)}{\Delta\left(l_{0}, l_{1}, l_{4}\right) \Delta\left(l_{1}, l_{2}, l_{5}\right) \Delta\left(l_{2}, l_{0}, l_{3}\right)}\right) .
$$

According to Theorem 1.3 of [GZ] $\mathcal{L}_{3}^{\mathcal{M}}$ is different from $\mathcal{L}_{3}^{G}$, and

$$
F_{3}\left(l_{0}, \ldots, l_{4}\right)=\frac{1}{9} \operatorname{Alt}_{5}\left(\log \left|\Delta\left(l_{0}, l_{1}, l_{2}\right)\right| \log \left|\Delta\left(l_{1}, l_{2}, l_{3}\right)\right| \log \left|\Delta\left(l_{2}, l_{3}, l_{4}\right)\right|\right) .
$$

(3) If $n>3$, then $\mathcal{L}_{n}^{\mathcal{M}}$ is different from $\mathcal{L}_{n}^{G}$ since it is already so for the restriction to the special configuration; see Theorem 4.5.

The space of the functional equations for the function $\mathcal{L}_{3}^{G}$ is smaller than the one for $\mathcal{L}_{3}^{\mathcal{M}}$; see Chapter 1.5 of [G3]. A similar situation is expected for all $n>3$.

The space of the functional equations for the motivic $n$-logarithm function $\mathcal{L}_{n}^{\mathcal{M}}$ should provide an explicit construction of the weight $n$ part of the motivic Lie coalgebra of an arbitrary field $F$, as explained in Section 4.1 in [G6], taking into account the following correction.

Correction. In Section 4.2 of [G6] the subgroup of the functional equations $\mathcal{R}_{n}^{G}$ is supposed to be defined as the subgroup of all functional equations for the function $\mathcal{L}_{n}^{\mathcal{M}}, \operatorname{not} \mathcal{L}_{n}^{G}$.

\section{The Chow DILOGARITHM AND A RECIPROCITY LAW}

The Chow dilogarithm provides a homomorphism $\Lambda^{3} \mathbb{C}(X)^{*} \rightarrow \mathbb{R}$ given by

$$
f_{1} \wedge f_{2} \wedge f_{3} \longmapsto \mathcal{P}_{2}\left(X ; f_{1}, f_{2}, f_{3}\right):=\frac{1}{2 \pi i} \int_{X(\mathbb{C})} r_{2}\left(f_{1}, f_{2}, f_{3}\right) .
$$

In this section we show that the Chow dilogarithm can be expressed by the function $\mathcal{L}_{2}(z)$. The precise versions of this claim are discussed below.

1. The set up ([G1], G2]). For any field $F$ we defined in [G1] the groups

$$
\mathcal{B}_{n}(F):=\frac{\mathbb{Z}\left[F^{*}\right]}{\mathcal{R}_{n}(F)}, \quad n \geq 2
$$

and homomorphisms

$$
\begin{aligned}
\delta_{n}: \mathcal{B}_{n}(F) \longrightarrow \mathcal{B}_{n-1}(F) \otimes F^{*} ; & \{x\}_{n} \longmapsto\{x\}_{n-1} \otimes x, \quad n \geq 3, \\
\delta_{2}: \mathcal{B}_{2}(F) \longrightarrow \Lambda^{2} F^{*}, & \{x\}_{2} \longmapsto(1-x) \wedge x .
\end{aligned}
$$

There is a complex $\Gamma(F ; n)$,

$$
\mathcal{B}_{n}(F) \stackrel{\delta_{n}}{\longrightarrow} \mathcal{B}_{n-1}(F) \otimes F^{*} \stackrel{\delta_{n}}{\rightarrow} \ldots \stackrel{\delta_{n}}{\rightarrow} \mathcal{B}_{2}(F) \otimes \Lambda^{n-2} F^{*} \stackrel{\delta_{n}}{\rightarrow} \Lambda^{n} F^{*}
$$

where $\delta_{n}\left(\{x\}_{k} \otimes Y\right):=\{x\}_{k-1} \otimes x \wedge Y$ for $k>2$, and $(1-x) \wedge x \wedge y$ for $k=2$, called the weight $n$ polylogarithmic complex.

If $K$ is a field with a discrete valuation $v$ and the residue field $k_{v}$, then there is a homomorphism of complexes $\operatorname{res}_{v}: \Gamma(K, n) \longrightarrow \Gamma\left(k_{v}, n-1\right)[-1]$ (see Subsection 14 of Section 1 in [G1]). For example, for $n=3$ we have

$$
\begin{aligned}
& \mathcal{B}_{3}(K) \stackrel{\delta_{3}}{\longrightarrow} \mathcal{B}_{2}(K) \otimes K^{*} \stackrel{\delta_{3}}{\longrightarrow} \Lambda^{3} K^{*} \\
& \downarrow \operatorname{res}_{v} \quad \downarrow \operatorname{res}_{v} \\
& \mathcal{B}_{2}\left(k_{v}\right) \quad \stackrel{\delta_{2}}{\longrightarrow} \Lambda^{2} k_{v}^{*}
\end{aligned}
$$


Here $\operatorname{res}_{v}\left(\{x\}_{2} \otimes y\right)$ is zero unless $v(x)=0$. In the latter case it is $\operatorname{res}_{v}\left(\{x\}_{2} \otimes y\right)=$ $v(y)\{\bar{x}\}_{2}$, where $\bar{x}$ denotes projection of $x$ to the residue field of $K$.

Let $X$ be a regular curve over an algebraically closed field $k$ and $F:=k(X)^{*}$. Set Res $:=\sum_{x} \operatorname{res}_{x}$ where $\operatorname{res}_{x}$ is the residue homomorphism for the valuation on $F$ corresponding to a point $x$ of $X$. For instance for $n=3$ we get a morphism of complexes

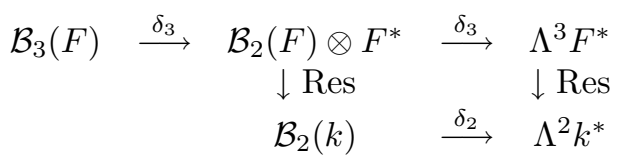

We will also need a more explicit version $B_{2}(F)$ of the group $\mathcal{B}_{2}(F)$. Denote by $R_{2}(F)$ the subgroup of $\mathbb{Z}\left[\mathbb{P}^{1}(F)\right]$ generated by the elements

$$
\{0\},\{\infty\} \text { and } \sum_{i=1}^{5}(-1)^{i}\left\{r\left(x_{1}, \ldots, \widehat{x}_{i}, \ldots, x_{5}\right)\right\}
$$

when $\left(x_{1}, \ldots, x_{5}\right)$ runs through all 5 -tuples of distinct points in $\mathbb{P}^{1}(F)$, and the cross-ratio $r(\ldots)$ is normalized by $r(\infty, 0,1, x)=x$. Define the Bloch group $B_{2}(F)$ as

$$
B_{2}(F):=\frac{\mathbb{Z}\left[\mathbb{P}^{1}(F)\right]}{R_{2}(F)} .
$$

One can show that $R_{2}(F) \subset \mathcal{R}_{2}(F)$. So there is a map

$$
i: B_{2}(k) \longrightarrow \mathcal{B}_{2}(k)
$$

induced by the identity map on the generators.

Proposition 6.1. Let $k$ be a number field. Then (78) is an isomorphism modulo torsion.

Proof. The map $i$ is clearly surjective. The diagram

$$
\begin{array}{crrr}
B_{2}(k) & \longrightarrow & \mathcal{B}_{2}(k) \\
\downarrow \delta_{2} & & \downarrow \delta_{2} \\
\Lambda^{2} k^{*} & = & \Lambda^{2} k^{*}
\end{array}
$$

is commutative. So we need only to show that if $0 \neq x \in B_{2}(k)_{\mathbb{Q}}$ and $\delta_{2}(x)=0$, then $i(x) \neq 0$. This follows from the injectivity of the regulator map on $K_{3}^{\text {ind }}(k)_{\mathbb{Q}}$. Indeed, by Suslin's theorem for a field $F$ one has $K_{3}^{\text {ind }}(F)_{\mathbb{Q}}=\operatorname{Ker} \delta_{2} \otimes \mathbb{Q}$. Let us identify $K_{3}^{\text {ind }}(\mathbb{C})_{\mathbb{Q}}$ with this subgroup of $B_{2}(\mathbb{C})_{\mathbb{Q}}$. The restriction of the dilogarithm map

$$
B_{2}(k) \longrightarrow \mathcal{B}_{2}(k) \longrightarrow(\mathbb{Z}[\operatorname{Hom}(k, \mathbb{C})] \otimes 2 \pi i \mathbb{R})^{+}, \quad\{z\}_{2} \longmapsto\left\{2 \pi i \mathcal{L}_{2}\left(\sigma_{i}(z)\right)\right\}
$$

to the subgroup $\operatorname{Ker} \delta_{2} \otimes \mathbb{Q}$ gives the Borel regulator $K_{3}^{\text {ind }}(k)_{\mathbb{Q}} \longrightarrow \mathbb{R}^{r_{2}}([\mathrm{G} 1])$ and thus is injective by Borel's theorem.

Remark. For any field $k$ the rigidity conjecture for $K_{3}^{\text {ind }}$ implies that the map $i$ should be an isomorphism; see [G1]. 


\section{The strong reciprocity law.}

Conjecture 6.2. Let $X$ be a regular projective curve over an algebraically closed field $k$ and $F:=k(X)^{*}$. Then there exists a canonical homomorphism of groups $h: \Lambda^{3} F^{*} \rightarrow \mathcal{B}_{2}(k)$ satisfying the following two conditions:

(a) $h\left(k^{*} \wedge \Lambda^{2} F^{*}\right)=0$ and the diagram

$$
\begin{array}{rrrr}
\mathcal{B}_{3}(F) \stackrel{\delta_{3}}{\longrightarrow} \mathcal{B}_{2}(F) \otimes F^{*} & \stackrel{\delta_{3}}{\longrightarrow} & \Lambda^{3} F^{*} \\
\operatorname{Res} \downarrow & h \swarrow & \downarrow \text { Res } \\
\mathcal{B}_{2}(k) & \stackrel{\delta_{2}}{\longrightarrow} & \Lambda^{2} k^{*}
\end{array}
$$

is commutative.

(b) If $X$ is a curve over $\mathbb{C}$, then

$$
\frac{1}{2 \pi i} \int_{X(\mathbb{C})} r_{2}\left(f_{1} \wedge f_{2} \wedge f_{3}\right)=\mathcal{L}_{2}\left(h\left(f_{1} \wedge f_{2} \wedge f_{3}\right)\right) .
$$

Remarks. (1) (b) follows easily if we have a functorial map $h$ such that $\operatorname{Res}=\delta_{2} \circ h$; see Theorem 6.10 below.

(2) According to Suslin's reciprocity law for the Milnor group $K_{3}^{M}(F)$ the projection of $\operatorname{Res}\left(\Lambda^{3} F^{*}\right) \subset \Lambda^{2} k^{*}$ to $K_{2}(k)$ is zero. Since by Matsumoto's theorem $K_{2}(k)=\operatorname{Coker}\left(\delta_{2}\right)$, one has $\operatorname{Res}\left(\Lambda^{3} F^{*}\right) \subset \operatorname{Im}\left(\delta_{2}\right)$. However $\operatorname{Ker}\left(\delta_{2}\right)$ is nontrivial, so it is a priori unclear that we can lift naturally the map Res to a map $h$. One of the reasons why we can do this is provided by (80).

We prove this conjecture in the following cases:

(a) $X=\mathbb{P}^{1}$; we construct explicitly a reciprocity homomorphism $h: \Lambda^{3} F^{*} \rightarrow$ $B_{2}(k)$ in Theorem 6.5

(b) $X$ is an elliptic curve over an algebraically closed field; we construct explicitly a reciprocity homomorphism $h: \Lambda^{3} F^{*} \rightarrow \mathcal{B}_{2}(k)$ in Theorem 6.14.

(c) $k=\overline{\mathbb{Q}}, X$ is any curve; see Theorem 6.12.

In the cases (a) and (b) the homomorphism $h$ satisfies the following additional property. Let $F=k(X)$ and $k$ is not necessarily algebraically closed. Let $k^{\prime}$ be the field of definition of the divisors $\left(f_{1}\right),\left(f_{2}\right),\left(f_{3}\right)$. Then $h\left(f_{1} \wedge f_{2} \wedge f_{3}\right) \in \mathcal{B}_{2}\left(k^{\prime}\right)$.

Conjecture 6.3. Let $X$ be a projective regular curve over an algebraically closed field $k$ and $F:=k(X)$. Then the homomorphism

$$
\text { Res : } \Gamma(F ; n) \longrightarrow \Gamma(k ; n-1)[-1]
$$

is homotopic to zero.

Lemma 6.4. Assume that we have a map $h$ such that $h\left(k^{*} \wedge \Lambda^{2} F^{*}\right)=0$ and Res $=\delta_{2} \circ h$. Then $h \circ \delta_{3}=$ Res.

Proof. The image of the group $\mathcal{B}_{2}(F) \otimes F^{*}$ under the map $h \circ \delta_{3}$ belongs to the subgroup $\operatorname{Ker} \delta_{2}$. Since $h\left(k^{*} \wedge \Lambda^{2} F^{*}\right)=0$ one has $h \circ \delta_{3}=\operatorname{Res}$ on $\mathcal{B}_{2}(F) \otimes k^{*}$. Any element of $k(X)^{*}$ can be connected via a curve to a constant. This together with the rigidity of $\operatorname{Ker} \delta_{2}$ (built into the definition of the group $\mathcal{B}_{2}(k)$ ) implies the result.

3. The $X=\mathbb{P}^{1}$ case. Recall that $v_{x}(f)$ is the order of zero of $f \in k(X)$ at $x$. Choose a point $\infty$ on $\mathbb{P}^{1}$. 
Theorem 6.5. Assume that $k=\bar{k}$. Then the map $h: \Lambda^{3} k\left(\mathbb{P}^{1}\right)^{*} \rightarrow B_{2}(k)$ given by the formula

$$
h\left(f_{1} \wedge f_{2} \wedge f_{3}\right):=\sum_{x_{i} \in \mathbb{P}^{1}(k)} v_{x_{1}}\left(f_{1}\right) v_{x_{2}}\left(f_{2}\right) v_{x_{3}}\left(f_{3}\right)\left\{r\left(x_{1}, x_{2}, x_{3}, \infty\right)\right\}_{2}
$$

satisfies all the conditions of Conjecture 6.2 modulo 6-torsion.

Proof. Let us show that $h$ is independent of the choice of $\infty$, i.e.,

$$
\sum_{x_{i} \in \mathbb{P}^{1}(k)} v_{x_{1}}\left(f_{1}\right) v_{x_{2}}\left(f_{2}\right) v_{x_{3}}\left(f_{3}\right)\left\{r\left(x_{1}, x_{2}, x_{3}, a\right)\right\}_{2} \in B_{2}(k)
$$

does not depend on $a$. Indeed, the 5 -term relation for the 5 -tuple of points $\left(x_{1}, x_{2}\right.$, $\left.x_{3}, a, b\right)$ gives

$$
\begin{gathered}
\sum_{x_{i} \in \mathbb{P}^{1}(k)} v_{x_{1}}\left(f_{1}\right) v_{x_{2}}\left(f_{2}\right) v_{x_{3}}\left(f_{3}\right)\left(\left\{r\left(x_{1}, x_{2}, x_{3}, a\right)\right\}_{2}-\left\{r\left(x_{1}, x_{2}, x_{3}, b\right)\right\}_{2}\right) \\
=-\sum_{x_{i} \in \mathbb{P}^{1}(k)} v_{x_{1}}\left(f_{1}\right) v_{x_{2}}\left(f_{2}\right) v_{x_{3}}\left(f_{3}\right)\left(\left\{r\left(x_{1}, x_{2}, a, b\right)\right\}_{2}\right. \\
\left.-\left\{r\left(x_{1}, x_{3}, a, b\right)\right\}_{2}+\left\{r\left(x_{2}, x_{3}, a, b\right)\right\}_{2}\right) .
\end{gathered}
$$

Each of these 3 terms vanishes because $\sum_{x \in \mathbb{P}^{1}(k)} v_{x}(f)=0$ for any $f \in k\left(\mathbb{P}^{1}\right)^{*}$.

Proposition 6.6. Let $k=\bar{k}$. Then modulo 6-torsion,

$$
h((1-f) \wedge f \wedge g)=\sum_{x \in \mathbb{P}^{1}(k)} v_{x}(g)\{f(x)\}_{2} .
$$

Proof. Using linearity with respect to $g$ and projective invariance of the cross-ratio we see that it is sufficient to prove the identity for $g=t$. Then it boils down to showing

$$
\sum_{x_{i} \in \mathbb{P}^{1}(k)} v_{x_{1}}(1-f) v_{x_{2}}(f)\left\{r\left(x_{1}, x_{2}, 0, \infty\right)\right\}_{2}=\{f(0)\}_{2}-\{f(\infty)\}_{2} .
$$

Lemma 6.7. Applying $\delta_{2}$ to both parts of 810 we get the same result modulo 6-torsion.

Proof. Choose a coordinate $t$ on $\mathbb{P}^{1}$ such that $f(\infty)=1$. Then

$$
f(t)=\frac{\prod_{i}\left(a_{i}-t\right)^{\alpha_{i}}}{\prod_{k}\left(c_{k}-t\right)^{\gamma_{k}}} ; \quad 1-f(t)=\frac{B \prod_{j}\left(b_{j}-t\right)^{\beta_{j}}}{\prod_{k}\left(c_{k}-t\right)^{\gamma_{k}}} .
$$

Observe that $\{f(\infty)\}_{2}=0$ modulo 6 -torsion. The left-hand side equals

$$
\begin{gathered}
\sum_{x_{i} \in \mathbb{P}^{1}(k)} v_{x_{1}}(1-f) v_{x_{2}}(f)\left\{x_{1} / x_{2}\right\}_{2} \\
=\sum \beta_{j} \alpha_{i}\left\{b_{j} / a_{i}\right\}_{2}-\sum \gamma_{k} \alpha_{i}\left\{c_{k} / a_{i}\right\}_{2}-\sum \beta_{j} \gamma_{k}\left\{b_{j} / c_{k}\right\}_{2} .
\end{gathered}
$$


Applying $\delta_{2}$ to it we get

$$
\begin{gathered}
\sum \beta_{j} \alpha_{i} \cdot \frac{a_{i}-b_{j}}{a_{i}} \wedge \frac{b_{j}}{a_{i}}-\sum \gamma_{k} \alpha_{i} \cdot \frac{a_{i}-c_{k}}{a_{i}} \wedge \frac{c_{k}}{a_{i}}-\sum \gamma_{k} \beta_{j} \cdot \frac{c_{k}-b_{j}}{c_{k}} \wedge \frac{b_{j}}{c_{k}} \\
=\sum \beta_{j} \alpha_{i} \cdot b_{j} \wedge a_{i}+\sum \alpha_{i} \gamma_{k} \cdot a_{i} \wedge c_{k}+\sum \gamma_{k} \beta_{j} \cdot c_{k} \wedge b_{j} \\
-\sum_{i} \frac{\prod_{j}\left(a_{i}-b_{j}\right)^{\beta_{j}}}{\prod_{k}\left(a_{i}-c_{k}\right)^{\gamma_{k}}} \wedge a_{i}^{\alpha_{i}}+\sum_{j} \frac{\prod_{i}\left(a_{i}-b_{j}\right)^{\alpha_{i}}}{\prod_{k}\left(c_{k}-b_{j}\right)^{\gamma_{k}}} \wedge b_{j}^{\beta_{j}}-\sum_{k} \frac{\prod\left(a_{i}-c_{k}\right)^{\alpha_{i}}}{\prod\left(c_{k}-b_{j}\right)^{\beta_{j}}} \wedge c_{k}^{\gamma_{k}} .
\end{gathered}
$$

Using (82) we see that the second line equals modulo 2-torsion

$$
-\sum_{i}\left(1-f\left(a_{i}\right)\right) \wedge a_{i}^{\alpha_{i}}+\sum_{j} f\left(b_{j}\right) \wedge b_{j}^{\beta_{i}}-\sum_{k} \frac{\prod\left(a_{i}-c_{k}\right)^{\alpha_{i}}}{\prod\left(b_{j}-c_{k}\right)^{\beta_{j}}} \wedge c_{k}^{\gamma_{k}}+B \wedge \prod a_{i}^{\alpha_{i}} .
$$

The first two terms are zero since $f\left(a_{i}\right)=0$ and $f\left(b_{j}\right)=1$. The third term equals $-\left(B \wedge \prod c_{k}^{\gamma_{k}}\right)$ since, as follows from (피), $f\left(c_{k}\right)=\infty$ and thus

$$
\frac{\prod\left(a_{i}-c_{k}\right)^{\alpha_{i}}}{\prod\left(b_{j}-c_{k}\right)^{\beta_{j}}}=-B
$$

On the other hand, we have

$$
\delta_{2}\left(\{f(0)\}_{2}\right)=(1-f(0)) \wedge f(0)=\frac{B \prod_{j} b_{j}^{\beta_{j}}}{\prod_{k} c_{k}^{\gamma_{k}}} \wedge \frac{\prod_{i} a_{i}^{\alpha_{i}}}{\prod_{k} c_{k}^{\gamma_{k}}},
$$

which matches the expression we obtained for the left-hand side. The lemma is proved.

To prove the proposition it remains to use a rigidity argument. Namely, we need to show that the identity is valid for some particular $f$, which is easy, or use Proposition 6.8 plus injectivity of the regulator on $K_{3}(\overline{\mathbb{Q}})_{\mathbb{Q}}$. The proposition is proved.

Now let us prove the key fact that Res $=\delta_{2} \circ h$. We need to show that for any 3 rational functions $f_{1}, f_{2}, f_{3}$ on $\mathbb{P}^{1}$,

$$
\begin{gathered}
\sum_{x \in \mathbb{P}^{1}(k)} \operatorname{res}_{x}\left(f_{1} \wedge f_{2} \wedge f_{3}\right) \\
=\delta_{2}\left(\sum_{x_{i} \in \mathbb{P}^{1}(k)} v_{x_{1}}\left(f_{1}\right) v_{x_{2}}\left(f_{2}\right) v_{x_{3}}\left(f_{3}\right)\left\{r\left(x_{1}, x_{2}, x_{3}, \infty\right)\right\}_{2}\right) .
\end{gathered}
$$

Both sides are obviously homomorphisms from $\Lambda^{3} F^{*}$ to $\Lambda^{2} k^{*}$ which are zero on $k^{*} \wedge \Lambda^{2} F^{*}$. (The last property for the map $\sum \operatorname{res}_{x}$ is provided by the Weil reciprocity law.) We normalize the cross-ratio of four points on the projective line by $r(\infty, 0,1, z)=z$. So it suffices to check the formula on elements

$$
\frac{z-a_{2}}{z-a_{1}} \wedge \frac{z-b_{2}}{z-b_{1}} \wedge \frac{z-c_{2}}{z-c_{1}}
$$

In this case it follows from

$$
\delta_{2}\left\{r\left(a_{2}, b_{2}, c_{2}, \infty\right)\right\}_{2}=\delta_{2}\left\{\frac{a_{2}-c_{2}}{b_{2}-c_{2}}\right\}_{2}=\frac{b_{2}-a_{2}}{b_{2}-c_{2}} \wedge \frac{a_{2}-c_{2}}{b_{2}-c_{2}}
$$

It remains to prove the following proposition. 


\section{Proposition 6.8.}

$$
\mathcal{P}_{2}\left(\mathbb{P}^{1} ; f_{1}, f_{2}, f_{3}\right)=\sum_{x_{i} \in \mathbb{P}^{1}(\mathbb{C})} v_{x_{1}}\left(f_{1}\right) v_{x_{2}}\left(f_{2}\right) v_{x_{3}}\left(f_{3}\right) \mathcal{L}_{2}\left(r\left(x_{1}, x_{2}, x_{3}, \infty\right)\right) .
$$

Proof. We immediately reduce the statement to the situation when $f_{1}=1-z, f_{2}=$ $z, f_{3}=z-a$, which is a particular case of the following lemma.

Lemma 6.9. Let $X$ be an arbitrary curve over $\mathbb{C}$. Then

$$
\int_{X(\mathbb{C})} r_{2}((1-f) \wedge f \wedge g)=-\sum_{x \in X(\mathbb{C})} v_{x}(g) \cdot \mathcal{L}_{2}(f(x)) .
$$

Proof. For functions $f(z)$ and $g(z)$ on $X(\mathbb{C})$ set

$$
\alpha(f, g):=\log |f| d \log |g|-\log |g| d \log |f| .
$$

Consider the following 1-form on $X(\mathbb{C})$ :

$$
\mathcal{L}_{2}(f) d \arg g-\frac{1}{3} \alpha(1-f, f) \log |g| \text {. }
$$

It defines a current on $X(\mathbb{C})$. We claim that its derivative is equal to

$$
2 \pi \cdot \mathcal{L}_{2}(f) \delta(g)+r_{2}((1-f) \wedge f \wedge g) .
$$

Using $d(d \arg g)=2 \pi \cdot \delta(g)$ and

$$
d \mathcal{L}_{2}(z)=-\log |1-z| d \arg z+\log |z| d \arg (1-z),
$$

we see that the differential of the current 85 equals

$$
\begin{gathered}
2 \pi \mathcal{L}_{2}(f) \delta(g)+(-\log |1-f| d \arg f+\log |f| d \arg (1-f)) \wedge d \arg g \\
+\frac{1}{3}(\log |1-f| d \log |f|-\log |f| d \log |1-f|) \wedge d \log |g| \\
-\frac{2}{3} \log |g| \cdot d \log |1-f| \wedge d \log |f| .
\end{gathered}
$$

Since $d \log (1-f) \wedge d \log f=0$ we have

$$
d \log |1-f| \wedge d \log |f|=d \arg (1-f) \wedge d \arg f .
$$

Using this and writing $r_{2}\left(f_{1} \wedge f_{2} \wedge f_{3}\right)$ as

$$
\begin{aligned}
& \frac{1}{3}\left(\log \left|f_{1}\right| d \log \left|f_{2}\right| \wedge d \log \left|f_{3}\right|+\text { cyclic permutations }\right) \\
& -\left(\log \left|f_{1}\right| d \arg \left(f_{2}\right) \wedge d \arg f_{3}+\text { cyclic permutations }\right)
\end{aligned}
$$

we come to (86). Integrating we get the lemma. The proposition is proved.

4. Expressing the Chow dilogarithm via the classical one. Let $\pi: Y \rightarrow S$ be a family of curves over a base $S$ over $\mathbb{C}$ and $f_{1}, f_{2}, f_{3} \in \mathbb{C}(Y)^{*}$. We get a function at the generic point of $S$. Its value at $s \in S$ is given by the Chow dilogarithm $\mathcal{P}_{2}\left(Y^{s} ; f_{1}^{s}, f_{2}^{s}, f_{3}^{s}\right)$, where $Y^{s}$ is the fiber of $\pi$ at $s$. Denote it by $\mathcal{P}_{2}\left(Y \rightarrow S ; f_{1}, f_{2}, f_{3}\right)$.

Theorem 6.10. (a) Let $\pi: Y \rightarrow S$ be a family of curves over a base $S$ over $\mathbb{C}$. Then there are rational functions $\varphi_{i}$ on $S$ such that

$$
\mathcal{P}_{2}\left(Y \rightarrow S ; f_{1}, f_{2}, f_{3}\right)=\sum_{i} \mathcal{L}_{2}\left(\varphi_{i}(s)\right)
$$


(b) Let $k=\mathbb{C}(S), X$ is the generic fiber of $\pi$, and $F=k(X)$. Suppose that there exists a map $h: \Lambda^{3} F^{*} \rightarrow \mathcal{B}_{2}(k)$ such that Res $=\delta_{2} \circ h$. Then

$$
d \mathcal{P}_{2}\left(Y \rightarrow S ; f_{1}, f_{2}, f_{3}\right)=d \mathcal{L}_{2}\left(h\left(f_{1}, f_{2}, f_{3}\right)\right) .
$$

Proof. (a) We use the existence of the transfer map on $K_{3}^{M}$ to reduce the statement to the case $X=\mathbb{P}^{1}$.

Choose a projection $p: X \rightarrow \mathbb{P}^{1}$. We may suppose without loss of generality that $p$ is a (ramified) Galois covering with the Galois group $G$. Indeed, let $p_{1}: Y \rightarrow X$ be such a covering that its composition with $p$ is a Galois covering. Indeed,

$$
\mathcal{P}_{2}\left(Y \longrightarrow S ; p_{1}^{*} f_{1}, p_{1}^{*} f_{2}, p_{1}^{*} f_{3}\right)=\frac{1}{\operatorname{deg} p_{1}} \mathcal{P}_{2}\left(X \longrightarrow S ; f_{1}, f_{2}, f_{3}\right) .
$$

Then $\sum_{g \in G} g^{*}\left\{f_{1}, f_{2}, f_{3}\right\} \in p^{*} K_{3}^{M}\left(k\left(\mathbb{P}^{1}\right)\right)$. It coincides with $p^{*}$ of the transfer of the element $\left\{f_{1}, f_{2}, f_{3}\right\} \in K_{3}^{M}(F)$. This means that there exist $s_{1}^{(i)}, s_{2}^{(i)}, s_{3}^{(i)} \in k\left(\mathbb{P}^{1}\right)$ and $g_{j}, h_{j} \in k(X)$ such that

$$
\sum_{g \in G} g^{*}\left(f_{1} \wedge f_{2} \wedge f_{3}\right)-p^{*} \sum_{i} s_{1}^{(i)} \wedge s_{2}^{(i)} \wedge s_{3}^{(i)}=\sum_{j}\left(1-g_{j}\right) \wedge g_{j} \wedge h_{j} .
$$

Therefore,

$$
\begin{gathered}
\mathcal{P}_{2}\left(Y \rightarrow S ; f_{1}, f_{2}, f_{3}\right)=\frac{1}{|G|} \sum_{i} \mathcal{P}_{2}\left(\mathbb{P}^{1} \times S \rightarrow S ; s_{1}^{(i)}, s_{2}^{(i)}, s_{3}^{(i)}\right) \\
+\sum_{j} \mathcal{P}_{2}\left(\mathbb{P}^{1} \times S \rightarrow S ;\left(1-g_{j}\right), g_{j}, h_{j}\right) .
\end{gathered}
$$

It remains to use Lemma 6.9 and Proposition 6.8. Part (a) of the theorem is proved.

(b) We need the following lemma.

\section{Lemma 6.11.}

$$
d \mathcal{P}_{2}\left(Y \rightarrow S ; f_{1}, f_{2}, f_{3}\right)=(2 \pi)^{-1} \cdot \operatorname{Alt}_{3}\left(v_{x}\left(f_{1}\right) \log \left|f_{2}(x)\right| d_{s} \arg f_{3}(x)\right) .
$$

Proof. Using $d d \log f=2 \pi i \delta(f)$ we get an equality of 3-currents on $Y$ :

$$
d r_{2}\left(f_{1}, f_{2}, f_{3}\right)=\pi_{3}\left(\frac{d f_{1}}{f_{1}} \wedge \frac{d f_{2}}{f_{2}} \wedge \frac{d f_{3}}{f_{3}}\right)+2 \pi \operatorname{Alt}_{3}\left(\delta\left(f_{1}\right) \log \left|f_{2}(x)\right| d \arg f_{3}(x)\right) .
$$

The second term in (91) is a 1-form on the divisor $D:=\bigcup_{i=1}^{3} \operatorname{div}\left(f_{i}\right)$ considered as a 3-current on $Y$. This 1 -form is the composition of the residue map

$$
\text { res : } \Lambda^{3} \mathbb{C}(Y)^{*} \longrightarrow \coprod_{X \in Y_{1}} \Lambda^{2} \mathbb{C}(X)^{*}
$$

with the map

$r_{1}: \Lambda^{2} \mathbb{C}(X)^{*} \longrightarrow \mathcal{A}^{1}(\operatorname{Spec}(\mathbb{C}(X))), \quad f \wedge g \longmapsto-2 \pi(\log |f| d \arg g-\log |g| d \arg f)$.

The push-forward of the first term in (91) vanishes (since the fibers are complex curves the push-down of any $(3,0)$-form to $S$ is zero). Integrating the second 3current in (91) along the fibers of $Y$ we get (90). The lemma is proved.

According to Lemma 6.11 and formula (87) for $d \mathcal{L}_{2}$, and using Res $=\delta_{2} \circ h$ we get the proof of part (b) of the theorem. 
Remark. The function $\mathcal{L}_{2}(z)$ is continuous on $\mathbb{C P}^{1}$. Therefore, part (a) of the theorem implies that the function $\mathcal{P}_{2}\left(Y \rightarrow S ; f_{1}, f_{2}, f_{3}\right)$ can be extended to a continuous function on $S$.

\section{Conjecture 6.2 for $\bar{k}=\overline{\mathbb{Q}}$.}

Theorem 6.12. Let $X$ be a regular projective curve over $\overline{\mathbb{Q}}$ and $F:=\overline{\mathbb{Q}}(X)$. Then there exists a homomorphism $h: \Lambda^{3} F^{*} \rightarrow B_{2}(\overline{\mathbb{Q}}) \otimes \mathbb{Q}$ as in conjecture 6.2 such that for any embedding $\sigma: \overline{\mathbb{Q}} \hookrightarrow \mathbb{C}$ one has

$$
\frac{1}{2 \pi i} \int_{X(\mathbb{C})} r_{2}\left(\sigma\left(f_{1} \wedge f_{2} \wedge f_{3}\right)\right)=\mathcal{L}_{2}\left(\sigma\left(h\left(f_{1} \wedge f_{2} \wedge f_{3}\right)\right)\right) .
$$

Proof. It is similar to the proof of Theorem 6.10, Choose a projection $p: X \rightarrow \mathbb{P}^{1}$. We may suppose that $p$ is a Galois covering with the Galois group $G$. Indeed, let $p_{1}: Y \rightarrow X$ be such a covering that its composition with $p$ is a Galois covering. Setting

we may suppose that $p$ is Galois.

$$
h\left(f_{1} \wedge f_{2} \wedge f_{3}\right):=h\left(\frac{1}{\operatorname{deg} p_{1}} \cdot p_{1}^{*}\left(f_{1} \wedge f_{2} \wedge f_{3}\right)\right)
$$

Then $\sum_{g \in G} g^{*}\left\{f_{1}, f_{2}, f_{3}\right\} \in p^{*} K_{3}^{M}\left(k\left(\mathbb{P}^{1}\right)\right)$. So there exist $s_{1}^{(i)}, s_{2}^{(i)}, s_{3}^{(i)} \in k\left(\mathbb{P}^{1}\right)$ and $g_{j}, h_{j} \in k(X)$ such that (89) holds. Set

$$
|G| \cdot h\left(f_{1} \wedge f_{2} \wedge f_{3}\right):=\sum_{i} h\left(s_{1}^{(i)} \wedge s_{2}^{(i)} \wedge s_{3}^{(i)}\right)+\sum_{j} \sum_{x \in X(\overline{\mathbb{Q}})}\left\{g_{j}(x)\right\}_{2} \cdot v_{x}\left(h_{j}\right) .
$$

Lemma 6.13. Suppose $\sum_{i}\left(1-f_{i}\right) \wedge f_{i} \wedge g_{i}=0$ in $\Lambda^{3} \overline{\mathbb{Q}}(X)^{*}$. Then

$$
\sum_{i} \sum_{x \in X(\overline{\mathbb{Q}})} v_{x}\left(g_{i}\right) \cdot\left\{f_{i}(x)\right\}_{2}=0 \quad \text { in the group } B_{2}(\overline{\mathbb{Q}}) \text {. }
$$

This lemma implies that $h$ is well-defined. Indeed, suppose that we have a different presentation:

$$
\sum_{g \in G} g^{*}\left\{f_{1}, f_{2}, f_{3}\right\}=p^{*} \sum_{k} \widetilde{s}_{1}^{(k)} \wedge \widetilde{s}_{2}^{(k)} \wedge \widetilde{s}_{3}^{(k)}+\sum_{j}\left(1-\widetilde{g}_{j}\right) \wedge \widetilde{g}_{j} \wedge \widetilde{h}_{j} .
$$

We need to show that

$$
\begin{aligned}
& h\left(\sum_{k} \widetilde{s}_{1}^{(k)} \wedge \widetilde{s}_{2}^{k)} \wedge \widetilde{s}_{3}^{(k)}-\sum_{i} s_{1}^{(i)} \wedge s_{2}^{(i)} \wedge s_{3}^{(i)}\right) \\
+ & \sum v_{x}\left(h_{j}\right)\left\{g_{j}(x)\right\}_{2}-\sum v_{x}\left(\widetilde{h}_{j}\right)\left\{\widetilde{g}_{j}(x)\right\}_{2}=0 .
\end{aligned}
$$

There exist $a_{j}, b_{j} \in k\left(\mathbb{P}^{1}\right)$ such that modulo $k^{*} \wedge \Lambda^{2} k\left(\mathbb{P}^{1}\right)^{*}$ one has

$$
\sum_{k} \widetilde{s}_{1}^{(k)} \wedge \widetilde{s}_{2}^{(k)} \wedge \widetilde{s}_{3}^{(k)}-\sum_{i} s_{1}^{(i)} \wedge s_{2}^{(i)} \wedge s_{3}^{(i)}-\sum_{j}\left(1-a_{j}\right) \wedge a_{j} \wedge b_{j}=0 .
$$

According to Theorem 6.5 the homomorphism $h$ for $\mathbb{P}^{1}$ annihilates the left-hand side. On the other hand,

$$
\begin{gathered}
p^{*}\left(\sum_{k} \widetilde{s}_{1}^{(k)} \wedge \widetilde{s}_{2}^{(k)} \wedge \widetilde{s}_{3}^{(k)}-\sum_{i} s_{1}^{(i)} \wedge s_{2}^{(i)} \wedge s_{3}^{(i)}\right) \\
-\sum_{j}\left(1-\widetilde{g}_{j}\right) \wedge \widetilde{g}_{j} \wedge \widetilde{h}_{j}+\sum_{j}\left(1-g_{j}\right) \wedge g_{j} \wedge h_{j}=0 .
\end{gathered}
$$


Using Lemma 6.13 we get (6). To get (92) we use Theorem 6.10 and notice that (94)

$$
\mathcal{P}_{2}\left(Y \rightarrow S ; f_{1}, f_{2}, f_{3}\right)=1 / m \cdot \mathcal{P}_{2}\left(Z \rightarrow S ; p_{1}^{*} f_{1}, p_{1}^{*} f_{2}, p_{1}^{*} f_{3}\right)
$$

Proof of Lemma 6.13. For a regular curve $X$ over an algebraically closed field $k$ there is a commutative diagram

$$
\begin{array}{ccc}
B_{2}(F) \otimes F^{*} & \stackrel{\delta_{3}}{\longrightarrow} & \Lambda^{3} F^{*} \\
\operatorname{Res} \downarrow & \downarrow \operatorname{Res} \\
B_{2}(k) \otimes \mathbb{Z}[X(k)] \stackrel{\delta_{2}}{\longrightarrow} & \Lambda^{2} k^{*} \otimes \mathbb{Z}[X(k)]
\end{array}
$$

Thus for any point $x$ of the curve $X$ the element $\sum_{i} v_{x}\left(g_{i}\right) \cdot\left\{f_{i}(x)\right\}_{2}$ lies in $\operatorname{Ker} \delta_{2}$. Therefore it defines an element $\gamma_{x} \in K_{3}(\overline{\mathbb{Q}})_{\mathbb{Q}}$.

For any embedding $\sigma: \overline{\mathbb{Q}} \hookrightarrow \mathbb{C}$ the value of the Borel regulator on $\sigma\left(\gamma_{x}\right)$ is equal to $\sum_{x \in X} v_{x}\left(g_{i}\right) \cdot \mathcal{L}_{2}\left(\sigma\left(f_{i}(x)\right)\right)$. So by Lemma 6.9 the value of the Borel regulator on $\sum_{x} \sigma\left(\gamma_{x}\right)$ is equal to $2 \pi \cdot \int_{\mathbb{C P}^{1}} r_{2}\left(\sum_{i}\left(1-f_{i}\right) \wedge f_{i} \wedge g_{i}\right)$ and hence it is zero by our assumption. So Borel's theorem implies that the element is also zero.

A similar argument using Lemma 6.13 shows that the homomorphism $h$ does not depend on the choice of the (finite) Galois extension of $\mathbb{Q}\left(\mathbb{P}^{1}\right)$ containing the field $\mathbb{Q}(X)$.

6. Explicit formulas for the reciprocity homomorphism $h$ and the Chow dilogarithm in the case of an elliptic curve. Let $E$ be an elliptic curve. We want to calculate the integral $\int_{E(\mathbb{C})} r_{2}\left(f_{1} \wedge f_{2} \wedge f_{3}\right)$. Let us suppose that $E$ is realized as a plane curve. Then any rational function $f$ on $E$ can be written as a ratio of products of linear homogeneous functions:

$$
f=\frac{l_{1} \cdot \ldots \cdot l_{k}}{l_{k+1} \cdot \ldots \cdot l_{2 k}} .
$$

So it is enough to calculate the integral $\int_{E(\mathbb{C})} r_{2}\left(l_{1} / l_{0} \wedge l_{2} / l_{0} \wedge l_{3} / l_{0}\right)$ where the $l_{i}$ are linear functions in homogeneous coordinates. We will do this in a more general setting.

Notation. Let $X$ be a plane algebraic curve and $l_{i}$ linear functions in homogeneous coordinates. Denote by $L_{i}$ the line $l_{i}=0$ in $\mathbb{P}^{2}$. Let $D_{i}$ be the divisor $L_{i} \cap X$. Set $l_{i j}:=L_{i} \cap L_{j}$. For three points $a, b, c$ and a divisor $D=\sum n_{i}\left(x_{i}\right)$ on a line we will use the following notation (see Figure 11):

$$
\{r(a, b, c, D)\}_{2}:=\sum_{i} n_{i}\left\{r\left(a, b, c, x_{i}\right)\right\}_{2} .
$$

Theorem 6.14. Let $E$ be an elliptic curve over an algebraically closed field $k$. Then there exists a homomorphism of groups $h: \Lambda^{3} F^{*} \rightarrow \mathcal{B}_{2}(k)$ such that for any linear homogeneous functions $l_{0}, \ldots, l_{3}$ one has

$$
h\left(l_{1} / l_{0} \wedge l_{2} / l_{0} \wedge l_{3} / l_{0}\right)=-\sum_{i=0}^{3}(-1)^{i}\left\{r\left(l_{i 0}, \ldots, \widehat{l}_{i i}, \ldots, l_{i 3}, D_{i}\right)\right\}_{2}
$$




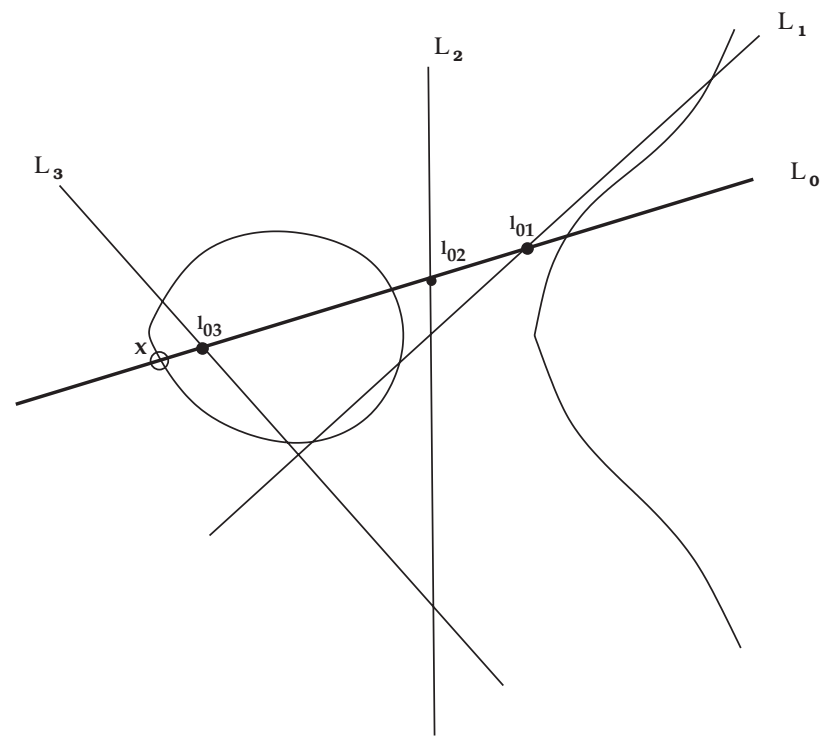

FiguRE 11. Defining $\{r(a, b, c, D)\}_{2}$ for a plane algebraic curve $X$

and which satisfies all the properties of Conjecture 6.2. In particular, if $k=\mathbb{C}$, then

$$
\mathcal{P}_{2}\left(E ; f_{1} \wedge f_{2} \wedge f_{3}\right)=\mathcal{L}_{2}\left(h\left(f_{1} \wedge f_{2} \wedge f_{3}\right)\right) .
$$

Proof. Suppose we have four generic lines $L_{0}, L_{1}, L_{2}, L_{3}$ in $\mathbb{P}^{2}$. Any two of them, say $L_{0}$ and $L_{1}$, provide a canonical rational function $\left(l_{0} / l_{1}\right)$ on $\mathbb{P}^{2}$ with the divisor $L_{0}-L_{1}$ normalized by the condition that its value at the point $l_{23}$ is equal to 1 .

Lemma 6.15. (a) On the line $L_{3}$ one has $\left(l_{1} / l_{0}\right)+\left(l_{2} / l_{0}\right)=1$.

$$
\text { (b) } \frac{\left(l_{1} / l_{0}\right)}{\left(l_{2} / l_{0}\right)}=-\left(l_{1} / l_{2}\right) \text {. }
$$

Proof. Let $m$ be a point on the line $L_{3}$. Then

$$
\left(l_{1} / l_{0}\right)(m)=r\left(l_{03}, l_{13}, l_{23}, m\right) ; \quad\left(l_{2} / l_{0}\right)(m)=r\left(l_{03}, l_{23}, l_{13}, m\right) .
$$

This gives (a). It follows from this that if the point $m$ approaches the point $l_{03}$, then $\frac{\left(l_{1} / l_{0}\right)}{\left(l_{2} / l_{0}\right)}$ tends to -1 . This implies (b).

Lemma 6.16. For any plane curve $X$ one has

$$
\sum_{x \in X} \operatorname{res}_{x}\left(\left(l_{1} / l_{0}\right) \wedge\left(l_{2} / l_{0}\right) \wedge\left(l_{3} / l_{0}\right)\right)=-\delta_{2}\left(\sum_{i=0}^{3}(-1)^{i}\left\{r\left(l_{i 0}, \ldots, \widehat{l}_{i i}, \ldots, l_{i 3}, D_{i}\right)\right\}_{2}\right) .
$$

Proof. Let us compute first the residues at the divisors $D_{1}, D_{2}, D_{3}$ using part (a) of Lemma 6.15. For example, the residue at $x \in D_{1}$ is equal to

$$
\begin{gathered}
v_{x}\left(\left(l_{1} / l_{0}\right)\right) \cdot\left(l_{2} / l_{0}\right)(x) \wedge\left(l_{3} / l_{0}\right)(x)=v_{x}\left(\left(l_{1} / l_{0}\right)\right) \cdot\left(1-\left(l_{3} / l_{0}\right)(x)\right) \wedge\left(l_{3} / l_{0}\right)(x) \\
\stackrel{97}{=} v_{x}\left(\left(l_{1} / l_{0}\right)\right) \cdot\left\{r\left(l_{13}, l_{10}, l_{12}, x\right)\right\}_{2}=v_{x}\left(\left(l_{1} / l_{0}\right)\right) \cdot\left\{r\left(l_{10}, l_{12}, l_{13}, x\right)\right\}_{2} .
\end{gathered}
$$


It remains to compute the residues on the line $L_{0}$. According to part (b) of Lemma 6.15 one has

$$
\left(l_{1} / l_{0}\right) \wedge\left(l_{2} / l_{0}\right) \wedge\left(l_{3} / l_{0}\right)=-\left(l_{0} / l_{1}\right) \wedge\left(l_{2} / l_{1}\right) \wedge\left(l_{3} / l_{1}\right) .
$$

Using this we reduce the calculation to the previous case.

Proposition 6.17. Let $E$ be an elliptic curve over an algebraically closed field $k$. Then formula (95) provides a well-defined homomorphism of groups $h: \Lambda^{3} F^{*} \rightarrow$ $\mathcal{B}_{2}(k)$.

Proof. Let $D:=\sum_{i} n_{i}\left(x_{i}\right)$ be the divisor of a rational function $f$ on $E$. To decompose it into a fraction of products of linear homogeneous functions $l_{i}$ we proceed as follows. Let $l_{x, y}$ (resp. $l_{x}$ ) be a linear homogeneous equation of the line in $\mathbb{P}^{2}$ through the points $x$ and $y$ on $E$ (resp. $x$ and $-x$ ). The divisor of the function $l_{x, y} / l_{x}$ is $(x)+(y)-(x+y)-(0)$. If $D=(x)+(y)+D_{1}$, we write $f=l_{x, y} / l_{x+y} \cdot f^{\prime}$, so $\left(f^{\prime}\right)=(0)+(x+y)+D_{1}$. After a finite number of such steps we get the desired decomposition. (See Figure[12])

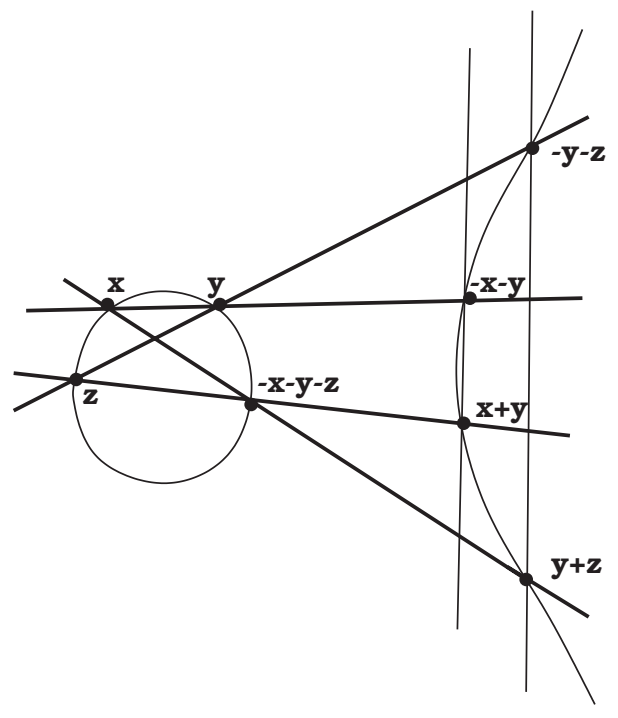

FiguRE 12.

There are the following relations:

$$
\frac{l_{x, y}}{l_{x+y}} \cdot \frac{l_{x+y, z}}{l_{x+y+z}} / \frac{l_{y, z}}{l_{y+z}} \cdot \frac{l_{x, y+z}}{l_{x+y+z}}=\text { constant. }
$$

One can prove that they generate all the relations between the functions $l_{x, y} / l_{x+y}$. So $h$ is well-defined if it annihilates the following expression:

$$
F\left(x, y, z ; l_{0}, l_{2}, l_{3}\right):=\left(\frac{l_{x, y}}{l_{x+y}} \cdot \frac{l_{x+y, z}}{l_{x+y+z}} / \frac{l_{y, z}}{l_{y+z}} \cdot \frac{l_{x, y+z}}{l_{x+y+z}}\right) \wedge\left(l_{2} / l_{0}\right) \wedge\left(l_{3} / l_{0}\right) .
$$

It follows from Lemma 6.16 that $\delta_{2}\left(F\left(x, y, z ; l_{0}, l_{2}, l_{3}\right)\right)=0$. Thus according to the definition of the group $\mathcal{B}_{2}(k)$ it is enough to check that $h\left(F\left(x^{\prime}, y^{\prime}, z^{\prime} ; l_{0}, l_{2}, l_{3}\right)\right)=0$ for a certain triple of points $\left(x^{\prime}, y^{\prime}, z^{\prime}\right)$. It is easy to see that $h\left(F\left(a, a, a ; l_{0}, l_{2}, l_{3}\right)\right)=$ 0 since then the first factor in $F$ is a constant. The proposition is proved. 
Proposition 6.18. Let $X$ be an algebraic curve in $\mathbb{P}^{2}$ over $\mathbb{C}$ and $l_{0}, \ldots, l_{3}$ linear homogeneous functions on $\mathbb{C}^{3}$. Then one has (using the notation defined above)

$$
\int_{X(\mathbb{C})} r_{2}\left(l_{1} / l_{0} \wedge l_{2} / l_{0} \wedge l_{3} / l_{0}\right)=2 \pi \cdot \sum_{i=0}^{3}(-1)^{i} \mathcal{L}_{2}\left(r\left(l_{i 0}, \ldots, \widehat{l}_{i i}, \ldots, l_{i 3}, D_{i}\right) .\right.
$$

Proof. It follows from Lemma 6.16 and Theorem 6.10 that the differentials of both sides coincide. So their difference is a constant. To show that this constant is zero we deform $X$ to a union of lines in $\mathbb{P}^{2}$. Using Proposition 6.8 one sees that formula (98) is valid when $X$ is a line in $\mathbb{P}^{2}$.

\section{APPENDIX: ON VOLUMES OF SIMPLICES IN SYMMETRIC SPACES}

1. Volumes of hyperbolic geodesic simplices as boundary integrals. A point $y$ of the $n$-dimensional hyperbolic space $\mathcal{H}_{n}$ defines a one-dimensional space $M_{y}$ of volume forms on the absolute $\partial \mathcal{H}_{n}$. It consists of the volume forms invariant under the action of the isotropy group of $y$. We write them as follows. Let $x_{0}, \ldots, x_{n}$ be the coordinates in a vector space $V_{n+1}$ of dimension $n+1$, and $Q(x):=x_{0}^{2}+\ldots+x_{n-1}^{2}-x_{n}^{2}$. Then $\mathcal{H}_{n}$ can be realized as the projectivization of the cone $Q(x)<0$, and its boundary is the projectivization of the cone $Q(x)=0$. Choose a point $y \in \mathcal{H}_{n}$. Lifting $y$ to a vector $y^{\prime} \in V_{n+1}$ we have the following volume form on the boundary $\partial \mathcal{H}_{n}$ :

$$
\frac{\delta(Q(x)) \sigma_{n+1}(x, d x)}{\left(x, y^{\prime}\right)^{n-1}} .
$$

If $y$ belongs to the boundary $\partial \mathcal{H}_{n}$, this formula provides a space $M_{y}$ of singular volume forms on the absolute; they are invariant under the isotropy group of $y$.

Let us choose for any point $y$ such a volume form $\mu_{y}$. For two points $x, y$ the ratio $\mu_{x} / \mu_{y}$ is a nonzero function on the absolute.

Let $I\left(y_{0}, \ldots, y_{n}\right)$ be the geodesic simplex with vertices at $y_{0}, \ldots, y_{n}$ where the points $y_{i}$ could be on the absolute. Denote by $\operatorname{vol} I\left(y_{0}, \ldots, y_{n}\right)$ the volume of this simplex with respect to the invariant volume form in $\mathcal{H}_{n}$ normalized by the following condition: if we realize the hyperbolic space as the interior of the unit ball $y_{1}^{2}+\ldots+$ $y_{n}^{2} \leq 1$, then the volume form restricted to the tangent space at the origin $(0, \ldots, 0)$ is $d y_{1} \wedge \ldots \wedge d y_{n}$.

Theorem 7.1. For any hyperbolic geodesic simplex $I\left(y_{0}, \ldots, y_{n}\right)$ one has (99)

$$
\frac{(n-1)^{n} \operatorname{vol}\left(S^{n-1}\right)}{n} \cdot \operatorname{vol} I\left(y_{0}, \ldots, y_{n}\right)=\int_{\partial \mathcal{H}_{n}} \log \left|\frac{\mu_{y_{1}}}{\mu_{y_{0}}}\right| d \log \left|\frac{\mu_{y_{2}}}{\mu_{y_{0}}}\right| \wedge \ldots \wedge d \log \left|\frac{\mu_{y_{n}}}{\mu_{y_{0}}}\right| .
$$

Let $\varphi\left(y_{0}, \ldots, y_{n}\right)$ be the function defined by the right-hand side of (99). Thanks to property (2) of Proposition 5.1 it does not depend on the choice of invariant volume forms $\mu_{y}$.

Proposition 7.2. The function $\varphi\left(y_{0}, \ldots, y_{n}\right)$ has the following properties: It is

(1) a smooth function on the vertices $y_{i}$;

(2) equal to zero if three of the vertices belong to the same geodesic;

(3) additive with respect to cutting of a simplex, i.e., if $y_{0}, \ldots, y_{n}$ are points such that $y_{0}, y_{1}, y_{2}$ are on the same geodesic, $y_{1}$ between $y_{0}$ and $y_{2}$, then

$$
\varphi\left(y_{0}, y_{2}, \ldots, y_{n+1}\right)=\varphi\left(y_{0}, y_{1}, y_{3}, \ldots, y_{n+1}\right)+\varphi\left(y_{1}, y_{2}, \ldots, y_{n+1}\right) ;
$$

(4) invariant under the action of the symmetry group $S O(n, 1)$. 
Proof. (1) This is clear.

(2) Let us realize the hyperbolic space as the interior part of the unit ball in $\mathbb{R}^{n}$. Consider the geodesic $l$ passing through the center of the ball in the vertical direction. The subgroup $S O(n-1) \subset S O(n, 1)$ preserves pointwise this geodesic. So for any point $y$ on the geodesic the invariant volume form $\mu_{y}$ is invariant under the action of the group $S O(n-1)$. The quotient of $\partial \mathcal{H}_{n}$ under the action of $S O(n-1)$ is given by the projection $p: \partial \mathcal{H}_{n} \longrightarrow \mathbb{R}$ onto the vertical axis. Take three points $y_{1}, y_{2}, y_{3}$ on the geodesic. Then $\frac{\mu_{y_{2}}}{\mu_{y_{1}}}$ and $\frac{\mu_{y_{3}}}{\mu_{y_{1}}}$ are lifted from the line $\mathbb{R}$. Therefore, $d \log \left|\frac{\mu_{y_{2}}}{\mu_{y_{1}}}\right| \wedge d \log \left|\frac{\mu_{y_{3}}}{\mu_{y_{1}}}\right|=0$. So for a degenerate simplex $\left(y_{0}, \ldots, y_{n}\right)$ one has

$$
\log \left|\frac{\mu_{y_{0}}}{\mu_{y_{1}}}\right| d \log \left|\frac{\mu_{y_{2}}}{\mu_{y_{1}}}\right| \wedge d \log \left|\frac{\mu_{y_{3}}}{\mu_{y_{1}}}\right| \wedge \ldots \wedge d \log \left|\frac{\mu_{y_{n}}}{\mu_{y_{1}}}\right|=0 .
$$

It remains to mention the skewsymmetry of the integral (99). Property (2) is proved.

(3) Follows from (2) and the additivity property from Proposition 5.1.

(4) This is clear from (4) of Proposition 5.1 The proposition is proved.

The leading term of the Taylor expansion of the function $\varphi\left(y_{0}, y_{1}, \ldots, y_{n}\right)$ when $y_{0}$ is fixed and $y_{1}, \ldots, y_{n}$ are near $y_{0}$ provides an exterior $n$-form in $T_{y_{0}} \mathcal{H}_{n}$ denoted $\varphi_{y_{0}}\left(Y_{1}, \ldots, Y_{n}\right), Y_{i} \in T_{y_{0}} \mathcal{H}_{n}$. Let us compare it with the volume form $\operatorname{Vol}_{y_{0}}\left(Y_{1}, \ldots, Y_{n}\right)$ in $T_{y_{0}} \mathcal{H}_{n}$ normalized as before Theorem 7.1.

Lemma 7.3. $\varphi_{y_{0}}\left(Y_{1}, \ldots, Y_{n}\right)=\frac{(n-1)^{n}}{n} \operatorname{vol}\left(S^{n-1}\right) \cdot \operatorname{Vol}_{y_{0}}\left(Y_{1}, \ldots, Y_{n}\right)$.

Proof. Below we abuse notation by writing $y$ for $y^{\prime}$. One has $\mu_{y_{1}} / \mu_{y_{2}}=\frac{\left(y_{2}, x\right)^{n-1}}{\left(y_{1}, x\right)^{n-1}}$. So

$$
\varphi\left(y_{0}, \ldots, y_{n}\right)=(n-1)^{n} \int_{\partial \mathcal{H}_{n}} \log \left|\frac{\left(y_{1}, x\right)}{\left(y_{0}, x\right)}\right| d \log \left|\frac{\left(y_{2}, x\right)}{\left(y_{0}, x\right)}\right| \wedge \ldots \wedge d \log \left|\frac{\left(y_{n}, x\right)}{\left(y_{0}, x\right)}\right| .
$$

Thus

$$
\varphi_{y_{0}}\left(Y_{1}, \ldots, Y_{n}\right)=(n-1)^{n} \int_{\partial \mathcal{H}_{n}}\left(Y_{1}, x\right) d\left(Y_{2}, x\right) \wedge \ldots \wedge d\left(Y_{n}, x\right) .
$$

To do the computation of this integral we may suppose that $y_{0}=(0, \ldots, 0,1)$, $Y_{i}=\frac{\partial}{\partial y_{i}}$, so $\left(Y_{i}, x\right)=x_{i}$. Then the last integral equals

$$
(n-1)^{n} \int_{S^{n-1}} x_{1} d x_{2} \wedge \ldots \wedge d x_{n}=\frac{(n-1)^{n}}{n} \operatorname{vol}\left(S^{n-1}\right)
$$

where $S^{n-1}$ is the sphere $x_{1}^{2}+\ldots+x_{n}^{2}=1$. The lemma follows.

Proof of Theorem 7.1. Let us suppose first that the points $x_{i}$ are inside of the hyperbolic space. The function $\varphi\left(x_{0}, \ldots, x_{n}\right)$ defines an $n$-density $\widetilde{\varphi}$ on $\mathcal{H}_{n}$. Namely, to define the integral $\widetilde{\varphi}$ over a simplex $M$ one has to subdivide it into small simplices and take the sum of the functions $\varphi$ corresponding to their vertices. When the simplices are getting smaller the limit exists and is by definition $\int_{M} \widetilde{\varphi}$. Here we used properties (1) - (3). More precisely, (1) and (2) imply that $\varphi$ defines an additive volume form on $\mathcal{H}_{n}$, and (3) (together with (1)) guarantees that this volume form is $\sigma$-additive.

The skewsymmetry property implies that $\widetilde{\varphi}$ is actually a differential $n$-form. It is invariant under the action of the group $S O(n, 1)$. Therefore it is proportional to the standard volume form. 
Now suppose that the vertices $x_{i}$ can be on the absolute. Then it is easy to see that the corresponding integral (53) is still convergent. Moreover, if the vertices of the geodesic simplex are in general position, then it is a continuous function of the vertices. This implies that the volume of an ideal geodesic simplex is finite (which is, of course, an elementary fact) and coincides with the corresponding integral (99).

Completely similar results are valid for the complex $n$-dimensional hyperbolic space $\mathcal{H}_{n}{ }^{\mathbb{C}}:=\left\{\left|z_{1}\right|+\ldots+\left|z_{n}\right|^{2}<1\right\}, z_{i} \in \mathbb{C}$, and the quaternionic hyperbolic space $\mathcal{H}_{n}{ }^{\mathbb{H}}:=\left\{\left|q_{1}\right|+\ldots+\left|q_{n}\right|^{2}<1\right\}$ ( $q_{i}$ are quaternions). Indeed, a point $x$ in each of these spaces defines an invariant volume form $\mu_{x}$ on the boundary.

2. Calculation of the volume of a three-dimensional ideal geodesic simplex. If $n=3$ the absolute can be identified with $\mathbb{C P}^{1}$, and for the ideal simplex with vertices at the points $\infty, 0,1, a$ on the absolute we get

$$
\begin{gathered}
\operatorname{vol}(I(\infty, 0,1, a))=3 c_{3} \cdot \int_{\mathbb{C P}^{1}}(\log |z| d \log |1-z|-\log |1-z| d \log |z|) \wedge d \log |z-a| \\
=3 c_{3} \cdot \int_{\mathbb{C P}^{1}}(\log |z| d \arg (1-z)-\log |1-z| d \arg (z)) \wedge d \arg (z-a)
\end{gathered}
$$

because $d \log (z-1) \wedge d \log (z-a)=0$. Here $\log f=\log |f|+i \arg (f)$. Using

$$
d \mathcal{L}_{2}(z)=\log |z| d \arg (1-z)-\log |1-z| d \arg (z)
$$

we rewrite the last integral as

$$
3 c_{3} \cdot \int_{\mathbb{C P}^{1}} d \mathcal{L}_{2}(z) \wedge d \arg (z-a) .
$$

Computing the differential in the sense of distributions we get

$$
d\left(\mathcal{L}_{2}(z) d \arg (z-a)\right)=2 \pi \cdot \mathcal{L}_{2}(z) \delta(z-a) d x d y+d \mathcal{L}_{2}(z) \wedge d \arg (z-a) .
$$

So the integral of the right-hand side over $\mathbb{C P}^{1}$ is zero, i.e., the integral (100) is equal to $-6 \pi c_{3} \cdot \mathcal{L}_{2}(a)\left(c_{3}=-1 /(6 \pi)\right)$.

3. Volumes of geodesic simplices in $S L_{n}(\mathbb{C}) / S U(n)$. Recall the invariant differential $(2 n-1)$-form $\omega_{D_{n}}$ in $\mathbb{H}_{n}$.

Question. Is it true that

$$
\operatorname{vol}_{\omega_{D_{n}}}\left(I\left(x_{0}, \ldots, x_{2 n-1}\right)\right)=\text { constant } \times \psi_{n}\left(x_{0}, \ldots, x_{2 n-1}\right) ?
$$

One can show, following the lines of Subsection 1 in Section 7, that the positive answer to this question is equivalent to the following statement: if $x_{0}, x_{1}, x_{2}$ are on the same geodesic, then $\psi_{n}\left(x_{0}, \ldots, x_{2 n-1}\right)=0$.

4. Another approach to Grassmannian polylogarithms. The following construction was suggested to the author during the Fall of 1989, independently, by M. Kontsevich and by J. Nekovář. A hyperplane $h$ in an $n$-dimensional complex vector space $V$ determines an arrow in the space of degenerate nonnegative definite Hermitian forms in $V$ consisting of the forms with the kernel $h$. Let $h_{1}, \ldots, h_{2 n}$ be hyperplanes in $V$. Let $C\left(h_{1}, \ldots, h_{2 n}\right)$ be projectivization of the convex hull of the arrays corresponding to these hyperplanes. It is a simplex in $\overline{\mathbb{H}}_{n}$. The idea is to integrate the form $\omega_{D_{n}}$ over this simplex. If $n=2$ this construction provides an ideal geodesic simplex in the Cayley realization of the hyperbolic space, given by the interior part of a ball in $\mathbb{R P}^{3}$. However, the convergence of this integral for $n>2$ has not yet been established, although it does not seem to be a very difficult problem. If the integral is convergent, we get a function on configurations of $2 n$ 
hyperplanes in $\mathbb{C} P^{n-1}$. It would be very interesting to investigate this construction further and compare it with our construction of the Grassmannian polylogarithms.

5. A $(2 n-1)$-cocycle of $G L(\mathbb{C})$. Consider an infinite-dimensional $\mathbb{C}$-vector space with a given basis $e_{1}, \ldots, e_{m}, \ldots$. The group $G L(\mathbb{C})$ is the group of automorphisms of this space moving only a finite number of basis vectors.

Let us describe the restriction of the cocycle to the subgroup $G L_{n+m}(\mathbb{C})$ acting on the subspace generated by the first $n+m$ vectors. Take $2 n$ elements $g_{1}, \ldots, g_{2 n}$ of this group. Consider the corresponding $2 n(m+1)$-tuples of vectors:

$$
g_{1}\left(e_{n}, \ldots, e_{n+m}\right), \quad \ldots, \quad g_{2 n}\left(e_{n}, \ldots, e_{n+m}\right) .
$$

The set of all $(m+1)$-tuples of vectors forms a vector space. Let $\Delta_{2 n-1}$ be the standard simplex $\sum_{j=1}^{2 n} \lambda_{j}=1$. Consider the set $C_{n}^{m} \subset \Delta_{2 n-1}$ consisting of $\left(\lambda_{1}, \ldots, \lambda_{2 n}\right)$ such that

$$
\sum_{i=1}^{2 n} \lambda_{i} \cdot g_{i}\left(e_{n}, \ldots, e_{n+m}\right)
$$

is an $(m+1)$-tuple of vectors not in generic position. It is a cycle of codimension $n$. The Chow polylogarithm function evaluated on it provides the desired (measurable) cocycle.

The cocycle property follows from the functional equation for the Chow polylogarithm and the following general fact. The set of those $\left(\lambda_{1}, \ldots, \lambda_{2 n+1}\right)$ such that $\sum_{i} \lambda_{i} \cdot g_{i}\left(e_{n}, \ldots, e_{n+m}\right)$ is an $(m+1)$-tuple of vectors not in generic position is also of codimension $n$.

The construction is consistent with the restriction to $G L_{n}$ just by definition.

Problem. Show that the cohomology class of this cocycle is nontrivial and proportional to the Borel class.

\section{ACKNOWLEDGEMENT}

I am grateful to Spencer Bloch for discussions of Arakelov motivic complexes. The results of this paper were discussed in my course at Brown University (Spring 1998), and at the Newton Institute (Cambridge, March 1998). I am grateful to participants for their interest and useful comments. I am very grateful to the referees for many comments which greatly improved the exposition.

During the work on this paper I enjoyed the hospitality and support of the MPI (Bonn) and IHES (Bures-sur-Yvette). I am grateful to these institutions. This work was supported by the NSF grants DMS-9800998 and DMS-0099390.

\section{REFERENCES}

[A] Arnold V.I.: Mathematical methods of classical mechanics. Springer-Verlag, 1978. MF 0474390

[B1] Beilinson A.A.: Higher regulators and values of L-functions, VINITI, 24 (1984), 181-238. MF 0760999 (86h:11103)

[B2] Beilinson A.A.: Height pairing between algebraic cycles, Springer Lect. Notes in Math. 1289, 1987. MR0902590 (89g:11052)

[B3] Beilinson A.A.: Notes on absolute Hodge cohomology. Applications of algebraic $K$-theory to algebraic geometry and number theory, Parts I, II (Boulder, Colo., 1983), 35-68, Contemp. Math., 55, Amer. Math. Soc., Providence, RI, 1986. MR.0862628 (87m:14019)

[BD] Beilinson A.A., Deligne P.: Interprétation motivique de la conjecture de Zagier in Symp. in Pure Math., v. 55, part 2, 1994, 23-41. MR1265552 (95a:19008)

[BMS] Beilinson A.A., MacPherson R., Schechtman, V.V.: Notes on motivic cohomology, Duke Math. J. 54 (1987), 679-710. MR0899412 (88f:14021) 
[Bl1] Bloch S.: Algebraic cycles and higher K-theory, Adv. in Math., (1986), v. 61, 267-304. MF 0852815 (88f:18010)

[Bl2] Bloch S.: The moving lemma for higher Chow groups, J. Alg. Geometry 3 (1994). MF, 1269719 (96c:14007)

[Bl3] Bloch S.: Algebraic cycles and the Beilinson conjectures, Contemp. Math. 58 (1986) no 1, 65-79. MR0860404 (88e:14006)

[BK] Bloch S., Kriz I.: Mixed Tate motives, Ann. of Math., 140 (1994) vol. 3, 557-605, 1992. MF 1307897 (96c:11062) MR.1307897 (96c:11062)

[Bo1] Borel A.: Stable real cohomology of arithmetic groups, Ann. Sci. Ec. Norm. Super., (4) 7 (1974), 235-272. MR0387496 (52:8338)

[Bo2] Borel A.: Cohomologie de $S L_{n}$ et valeurs de fonctions zêta aux points entiers, Ann. Sc. Norm. Sup. Pisa 4 (1977), 613-636. MR0506168 (58:22016)

[Bott] Bott R.: On the characteristic classes of groups of diffeomorphisms, Ensiegnment Mathématique, T. XXIII, fsc. 3-4 (1977) 209-220. MR0488080 (58:7651)

[Bu] Burgos, J. I.: Arithmetic Chow rings and Deligne-Beilinson cohomology, J. Algebraic Geom. 6 (1997), no. 2, 335-377. MR.1489119 (99d:14015)

[C] Consani C.: Double complexes and Euler's L-factors, Compositio Math. 111 (1998) 323358. MR 1617133 (99b:11065)

[D] Dupont J.: Simplicial de Rham cohomolgy and characteristic classes of flat bundles, Topology 15 (1976), 233-245. MR0413122 (54:1243)

[Del] Deligne, P.: Le déterminant de la cohomologie. Current trends in arithmetical algebraic geometry (Arcata, Calif., 1985), 93-177, Contemp. Math., 67, Amer. Math. Soc., Providence, RI, 1987. MR0902592 (89b:32038)

[F] Fulton W.: Intersection theory. Second edition. Springer 1998. MR1644323 (99d:14003)

[Dyn] Dynkin E.B.: Topological characteristics of compact Lie groups homomorphisms, Math. Sb. NS 35 (1954), 129-173. MR.0067124 (16:673b)

[GGL] Gabrielov A., Gelfand I.M., Losik M.: Combinatorial computation of characteristic classes I, II, Funkt. Anal. i Pril. 9 (1975) 12-28, ibid 9 (1975) 5-26. MR0410758 $(53: 14504 \mathrm{a})$

[GM] Gelfand I.M., MacPherson R.: Geometry in Grassmannians and a generalisation of the dilogarithm, Adv. in Math., 44 (1982) 279-312. MR0658730 (84b:57014)

[GS] Gillet, H.; Soulé, C.: Arithmetic intersection theory, Inst. Hautes Études Sci. Publ. Math. No. 72, (1990), 93-174 (1991). MR1087394 (92d:14016)

[G1] Goncharov A.B.: Geometry of configurations, polylogarithms and motivic cohomology, Adv. in Math., 114, (1995), 197-318. MR1348706 (96g:19005)

[G2] Goncharov A.B.: Polylogarithms and motivic Galois groups in Symp. in Pure Math., v. 55, part 2, 1994, 43 - 97. MR 1265551 (94m:19003)

[G3] Goncharov A.B.: Explicit construction of characteristic classes, I.M. Gelfand seminar Adv. Soviet Math., 1993, v. 16, 169-210. MR1237830 (95c:57045)

[G4] Goncharov, A.B.: Deninger's conjecture on special values of L-functions of elliptic curves at $s=3$, J. Math. Sci. 81 (1996), N3, 2631-2656, alg-geom/9512016. MR1420221 (98c:19002)

[G5] Goncharov A.B.: Chow polylogarithms and regulators. Math. Res. Letters, 2, (1995), 99-114. MR,1312980 (96b:19007)

[G6] Goncharov, A.B.: Geometry of trilogarithm and the motivic Lie algebra of a field, Regulators in analysis, geometry and number theory, 127-165, Progr. Math., 171, Birkhäuser Boston, Boston, MA, 2000. math.AG/0011168. MR.1724890(2000j:11096)

[G7] Goncharov, A.B.: Explicit regulator maps on the polylogarithmic motivic complexes, Motives, polylogarithms and Hodge theory, Part I (Irvine, CA, 1998), 245-276, Int. Press Lect. Ser., 3, I, Int. Press, Somerville, MA, 2002. math.AG/0003086. MR1978709 (2004b:11095)

[GZ] Goncharov A.B., Zhao J.: The Grassmannian trilogarithms. Compositio Math. 127 (2001), no. 1, 83-108. math.AG/0011165. MR.1832988 (2002g:33004)

[HM] Hain R., MacPherson R.: Higher Logarithms, Ill. J. of Math,, vol. 34, (1990) N2, 392-475. MF 1046570 (92c:14016)

[H] Hain R.: The existence of higher logarithms. Compositio Math. 100 (1996) N3, 247- 276. MF.1387666 (97g:19008) 
[HY] Hain R., Yang R.: Real Grassmann polylogarithms and Chern classes. Math. Annalen, 304, (1996), N1, 157-201. MR1367888 (96k:19007)

[HM1] Hanamura M., MacPherson R.: Geometric construction of polylogarithms, Duke Math. J. 70 (1993) 481-516. MR1224097 (94h:19005)

[HM2] Hanamura M., MacPherson R.: Geometric construction of polylogarithms, II, Progress in Math. vol. 132 (1996) 215-282. MR1389020 (97g:19007)

[Lei] Gerhardt C.I. (ed). G.W.Leibniz Mathematische Schriften III/1 pp. 336-339 Georg Olms Verlag, Hildesheim. New York 1971. MR0141576 (25:4979b)

[Le] Levin A.M.: Notes on $\mathbb{R}$-Hodge-Tate sheaves. Preprint MPI 2001.

[Lev] Levine, M.: Bloch's higher Chow groups revisited. K-theory. Astérisque No. 226 (1994), 10, 235-320. MR1317122 (96c:14008)

[Li] Lichtenbaum S.: Values of zeta functions at non-negative integers, Lect. Notes in Math., 1068, Springer-Verlag, 1984, 127-138. MR0406981 (53:10765)

[M] MacPherson R.: Gabrielov, Gelfand, Losik combinatorial formula for the first Pontrjagin class Seminar Bourbaki 1976. MR0521763 (81a:57022)

[N] Nekováŕ J.: Beilinson's conjectures. Motives (Seattle, WA, 1991), 537-570, Proc. Sympos. Pure Math., 55, Part 1, Amer. Math. Soc., Providence, RI, 1994. Mr,1265544|(95f:11040)

[NS] Nesterenko Yu.P., Suslin A.A.: Homology of the general linear group over a local ring, and Milnor's K-theory. Math. USSR-Izv. 34 (1990), no. 1, 121-142. MR0992981 (90a:20092)

[RSS] Rapoport M., Schappacher N., Schneider P.: Beilinson's conjectures on special values of $L$-functions. Perspectives in Mathematics, 4. Academic Press, Inc., Boston, MA, 1988. MF 0944987 (89a:14002)

[Sch] Scholl A.: Integral elements in $K$-theory and products of modular curves. The arithmetic and geometry of algebraic cycles (Banff, AB, 1998), 467-489, NATO Sci. Ser. C Math. Phys. Sci., 548. MR1744957 (2001i:11077)

[S] Soulé C., Abramovich D., Burnol J. F., Kramer, J. K.: Lectures on Arakelov geometry. Cambridge University Press, 1992. MR1208731 (94e:14031)

[Su] Suslin A.A: Homology of $G L_{n}$, characteristic classes and Milnor's K-theory. Lect. Notes in Math. 1046 (1989), 357-375. MR0752941 (86f:11090b)

[Z1] Zagier D: Polylogarithms, Dedekind zeta functions and the algebraic $K$-theory of fields, Arithmetic Algebraic Geometry (G.v.d.Geer, F.Oort, J.Steenbrink, eds.), Prog. Math., Vol 89, Birkhäuser, Boston, 1991, pp. 391-430. MR1085270 (92f:11161)

Department of Mathematics, Brown University, Providence, Rhode Island 02912

E-mail address: sasha@math.brown.edu 